\title{
A REFINEMENT OF ANALYTIC CHARACTERIZATIONS OF GAUGEABILITY FOR GENERALIZED FEYNMAN-KAC FUNCTIONALS
}

\author{
DAEHONG KIM, MILA KURNIAWATY AND KAZUHIRO KUWAE
}

\begin{abstract}
We relax the conditions for measures in our previous paper [Analytic characterizations of gaugeability for generalized Feynman-Kac functionals (2016) Preprint] on analytic characterizations of (conditional) gaugeability for generalized FeynmanKac functionals in the framework of symmetric Markov processes. The analytic characterization is also equivalent to the maximum principle for generalized Feynman-Kac semigroups, extending the result by Takeda [The bottom of the spectrum of timechanged processes and the maximum principle of Schrödinger operators (2015) Preprint].
\end{abstract}

\section{Introduction}

In this paper, we relax the conditions for measures in [26, Theorems 1.1 and 1.2] on analytic characterizations of gaugeability or conditional gaugeability for generalized Feynman-Kac functionals in the framework of symmetric Markov processes. For this, let us state the framework of this paper. Let $E$ be a locally compact separable metric space and $\mathfrak{m}$ a positive Radon measure on $E$ with full topological support. Let $\mathbf{X}=\left(\Omega, \mathcal{F}_{\infty}, \mathcal{F}_{t}, X_{t}, \mathbf{P}_{x}, x \in E_{\partial}\right)$ be an $\mathfrak{m}$-symmetric transient Hunt process on $E$ with lifetime $\zeta:=\inf \left\{t>0 \mid X_{t}=\partial\right\}$. We assume that $\mathbf{X}$ satisfies the irreducibility condition $(\mathbf{I})$ and the absolute continuity condition (AC) (see Section 2 for the definitions). Then we can consider the Green function $R(x, y)$ of $\mathbf{X}$ defined on $E \times E$. We set $\mathrm{d}:=\{(x, y) \in E \times E \mid R(x, y)=0$ or $\infty\}$ the diagonal set with respect

Received October 3, 2015; received in final form June 7, 2016.

The first named author was partially supported by a Grant-in-Aid for Scientific Research (C) No. 23540147 from Japan Society for the Promotion of Science.

2010 Mathematics Subject Classification. Primary 31C25, 60J45, 60J57. Secondary 35J10, 60J35, 60J25. 
to $\mathbf{X}$ and $E^{x}:=\{y \in E \mid(x, y) \in(E \times E) \backslash \mathrm{d}\}$. Given an additive functional $A$ of $\mathbf{X}$, the expectation $g_{A}(x):=\mathbf{E}_{x}\left[e_{A}(\zeta)\right]$ (resp. conditional expectation $\left.g_{A}^{y}(x):=\mathbf{E}_{x}^{y}\left[e_{A}\left(\zeta^{y}\right)\right]\right)$ of the Feynman-Kac transform $e_{A}(t):=\exp \left(A_{t}\right)$ is called the gauge function (resp. the conditional gauge function, where $\mathbf{P}_{x}^{y}$ denotes the probability of Doob's $R(\cdot, y)$-transformed process starting from $x \in E)$.

In a series of papers, Chen and Song [9], [10] and Chen [4] have made remarkable progress in establishing the gauge and conditional gauge theorems for $A$ under quite general hypotheses on $\mathbf{X}$ and $A$ (in their papers, $\mathbf{X}$ is not necessarily symmetric). The gauge theorem asserts that under suitable conditions on $\mathbf{X}$ and $A$, the gauge function $g_{A}$ is either identically infinite or bounded on $E$. If the latter happens, $(\mathbf{X}, A)$ is said to be gaugeable. It is known that the conditional gauge theorem is related to the gauge theorem but is much deeper. The conditional gauge theorem asserts that under suitable conditions on $\mathbf{X}$ and $A$, the conditional gauge function $(x, y) \mapsto g_{A}^{y}(x)$ is either identically infinite or bounded between two positive constants. If the latter happens, $(\mathbf{X}, A)$ is said to be conditionally gaugeable and in this case, the Green function $R^{A}(x, y)$ of the Feynman-Kac transformed process $\mathbf{X}^{A}$ of $\mathbf{X}$ by $e_{A}(t)$ and $R(x, y)$ are comparable. So the infinitesimal generators of $\mathbf{X}^{A}$ and $\mathbf{X}$ share many potential theoretic properties. In this sense, it is important to know when $(\mathbf{X}, A)$ is conditionally gaugeable. Chen [4] introduced new classes of Kato class measures as genuine extensions of the Green-tight measure introduced by Zhao [45] and established various equivalent conditions for gaugeability and conditional gaugeability for a large class of processes and additive functionals related to possibly singular measures. In [26], the first and third authors introduced more natural (extended) Kato classes of (semi-)Green-tight measures including the (semi-)Green-tight measures of (extended) Kato class introduced by Chen [4]. These classes are stable under Girsanov transforms in some sense, which enables us to prove [26, Theorem 1.1]. In this paper, we also prove that the new class of Greentight measures of Kato class coincides with the class given by Chen [4] under the doubly resolvent Feller property (see Proposition 4.1).

Analytic characterizations of the gaugeability and conditional gaugeability for $(\mathbf{X}, A)$ have been studied by several authors. In the case of Brownian motion in $\mathbb{R}^{d}$, Zhao [45] introduced a so-called shuttle operator and showed that $(\mathbf{X}, A)$ is conditionally gaugeable if and only if the spectral bound of the shuttle operator is less than one. For more general symmetric Markov processes, Takeda [37] (resp. Chen [4]) gave an analytic characterization for a positive continuous additive functional (resp. a continuous additive functional) $A$ associated with a positive smooth measure (resp. a signed smooth measure) by using the $L^{p}$-independence of the spectral bound of $\mathbf{X}$ based on a large deviation principle (resp. a conditional gauge theorem) and later, this characterization was extended to non-local cases by Chen [5]. These analytic characterizations are not only useful in confirming whether $(\mathbf{X}, A)$ is gaugeable 
or conditionally gaugeable in many concrete cases, they are also applicable in studying subcriticality of Schrödinger operators, differentiability of spectral functions, stability of heat kernels under Feynman-Kac perturbations and certain penalization problems (see [37], [38], [40], [39], [43], [44]).

Now we give a detailed description of the results with necessary notations. Let $\mu_{1}$ (resp. $\mu_{2}$ ) be smooth measures in the strict sense corresponding to a positive continuous additive functional $A^{\mu_{1}}$ (resp. $A^{\mu_{2}}$ ) in the strict sense with respect to $\mathbf{X}$ and let $\mu:=\mu_{1}-\mu_{2}$ be the signed smooth measure in the strict sense. We denote $A$ by $A^{\mu}:=A^{\mu_{1}}-A^{\mu_{2}}$ to emphasize the correspondence between $\mu$ and $A$. Let $(\mathcal{E}, \mathcal{F})$ be the Dirichlet form of $\mathbf{X}$ on $L^{2}(E ; \mathfrak{m})$. For a bounded finely continuous (nearly) Borel function $u$ on $E$ which is strictly $\mathcal{E}$-quasi-continuous on $E_{\partial}$ and locally in $\mathcal{F}$, let $N^{u}$ be the continuous additive functional of zero quadratic variation appeared in the Fukushima decomposition of $u\left(X_{t}\right)-u\left(X_{0}\right)$ (see (2.2)). Note that $N^{u}$ is not necessarily of bounded variation in general. Let $F_{1}, F_{2}$ be non-negative bounded functions on $E \times E_{\partial}$ which are symmetric on $E \times E . F_{1}$ and $F_{2}$ are extended to $E_{\partial} \times E_{\partial}$ by setting $F_{i}(\partial, x)=F_{i}(x, \partial)=F_{i}(x, x)=0$ for $x \in E_{\partial}$ for each $i=1,2$ (actually there is no need to define the value $F_{i}(\partial, x)$ for $\left.x \in E, i=1,2\right)$. We set $F:=F_{1}-F_{2}$. Then $A_{t}^{F}:=\sum_{0<s \leq t} F\left(X_{s-}, X_{s}\right)$ (whenever it is summable) is an additive functional of $\mathbf{X}$. It is natural to consider the following generalized non-local Feynman-Kac transforms by the additive functionals $A:=N^{u}+A^{\mu}+A^{F}$ of the form

$$
e_{A}(t):=\exp \left(A_{t}\right), \quad t \geq 0,
$$

because the process $\mathbf{X}$ admits many continuous additive functionals which do not have bounded variations, and many discontinuous additive functionals. The purpose of this paper is to give analytic characterizations of the gaugeability and (semi-)conditional gaugeability for the generalized FeynmanKac transforms (1.1) which extend almost all the results in [5], [37], [44]. In particular, our result improves the previous work due to Chen [5] even if we restrict ourselves to deal with only non-local perturbations. More precisely, let $\mathcal{Q}$ be the quadratic form associated with the symmetric semigroup $P_{t}^{A} f(x):=\mathbf{E}_{x}\left[e_{A}(t) f\left(X_{t}\right)\right]$ (see [13]), which is defined by

$$
\mathcal{Q}(f, g):=\mathcal{E}(f, g)+\mathcal{E}(u, f g)-\mathcal{H}(f, g),
$$

where

$$
\begin{aligned}
\mathcal{E}(u, f g) & :=\frac{1}{2} \int_{E} f \mathrm{~d} \mu_{\langle u, g\rangle}+\frac{1}{2} \int_{E} g \mathrm{~d} \mu_{\langle u, f\rangle}, \\
\mathcal{H}(f, g) & :=\int_{E} f(x) g(x) \mu(\mathrm{d} x)+\int_{E} \int_{E} f(x) g(y)\left(e^{F(x, y)}-1\right) N(x, \mathrm{~d} y) \mu_{H}(\mathrm{~d} x) .
\end{aligned}
$$

$\mathcal{Q}(f, g)$ is well-defined for $f, g \in \mathcal{F}$ provided $\mu_{1}+N\left(F_{1}\right) \mu_{H}+\mu_{\langle u\rangle} \in S_{D}^{1}(\mathbf{X})$ and $\mu_{2}+N\left(F_{2}\right) \mu_{H} \in S_{D}^{1}(\mathbf{X})$ and is bounded from below on $L^{2}(E ; \mathfrak{m})$ provided $\mu_{1}+N\left(e^{F_{1}}-1\right) \mu_{H} \in S_{\mathrm{EK}}^{1}(\mathbf{X}), \mu_{\langle u\rangle} \in S_{K}^{1}(\mathbf{X})$ and $\mu_{2}+N\left(F_{2}\right) \mu_{H} \in S_{D}^{1}(\mathbf{X})$. 
Here $S_{\mathrm{EK}}^{1}(\mathbf{X})$ (resp. $\left.S_{K}^{1}(\mathbf{X})\right)$ denotes the class of smooth measures in the strict sense of extended Kato class (resp. Kato class) with respect to $\mathbf{X}$.

For a positive Radon measure $\eta$ on $E$, let $\lambda^{\mathcal{Q}}(\eta)$ be the bottom of the spectrum for $\mathcal{Q}$ on $L^{2}(E ; \eta)$ defined by

$$
\lambda^{\mathcal{Q}}(\eta):=\inf \left\{\mathcal{Q}(f, f) \mid f \in \mathcal{C}, \int_{E} f^{2} \mathrm{~d} \eta=1\right\},
$$

where $\mathcal{C}$ is the special standard core of $(\mathcal{E}, \mathcal{F})$ (see [19]). Let $S_{\mathrm{NK}_{\infty}}^{1}(\mathbf{X})$ (resp. $S_{\mathrm{NK}_{1}}^{1}(\mathbf{X})$ ) be the family of natural Green-tight measures of Kato class (resp. natural semi-Green-tight measures of extended Kato class) with respect to $\mathbf{X}$ and $S_{D_{0}}^{1}(\mathbf{X})$ the family of Green-bounded smooth measures with respect to $\mathbf{X}$ (see Section 4 for the definitions of these families). Let $(N, H)$ be the Lévy system of $\mathbf{X}$. We set $\bar{\mu}_{1}:=\mu_{1}+N\left(e^{F^{u}}-F^{u}-1+F_{1}\right) \mu_{H}+\frac{1}{2} \mu_{\langle u\rangle}^{c}$ and $\bar{\mu}_{1}^{*}:=\mu_{1}+N\left(e^{F_{1}^{u}}-F_{1}^{u}-1+F_{1}\right) \mu_{H}+\frac{1}{2} \mu_{\langle u\rangle}^{c}$, where $F^{u}(x, y):=F(x, y)+$ $U(x, y), F_{1}^{u}(x, y):=F_{1}(x, y)+U(x, y)$ and $U(x, y)=u(x)-u(y)$ for $x, y \in E_{\partial}$, and $\mu_{\langle u\rangle}^{c}$ is the continuous part of the energy measure of $u$.

The first main result of this paper is the following:

ThEOREM 1.1. Suppose that $\bar{\mu}_{1}^{*} \in S_{\mathrm{NK}_{1}}^{1}(\mathbf{X}), \mu_{\langle u\rangle} \in S_{\mathrm{NK}_{\infty}}^{1}(\mathbf{X})$ and $\mu_{2}+$ $N\left(F_{2}\right) \mu_{H} \in S_{D_{0}}^{1}(\mathbf{X})$ hold. Then $\lambda^{\mathcal{Q}}\left(\bar{\mu}_{1}\right)>0$ if and only if the functional (1.1) is gaugeable, i.e., $\sup _{x \in E} \mathbf{E}_{x}\left[e_{A}(\zeta)\right]<\infty$.

REMARK 1.1. Note that the condition $\bar{\mu}_{1}^{*} \in S_{\mathrm{NK}_{1}}^{1}(\mathbf{X})$ is equivalent to $\mu_{1}+$ $N\left(e^{U}\left(e^{F_{1}}-1\right)\right) \mu_{H} \in S_{\mathrm{NK}_{1}}^{1}(\mathbf{X})$ under $\mu_{\langle u\rangle} \in S_{\mathrm{NK}_{\infty}}^{1}(\mathbf{X})$. In [26, Theorem 1.1], we obtain the same conclusion of our Theorem 1.1 under the conditions $\mu_{1} \in$ $S_{\mathrm{NK}_{1}}^{1}(\mathbf{X}), \mu_{\langle u\rangle}+N\left(F_{1}\right) \mu_{H} \in S_{\mathrm{NK}_{\infty}}^{1}(\mathbf{X})$ and $\mu_{2}+N\left(F_{2}\right) \mu_{H} \in S_{D_{0}}^{1}(\mathbf{X})$. Our conditions for measures in Theorem 1.1 are milder than them.

Let us denote by $S_{\mathrm{CK}_{\infty}}^{1}(\mathbf{X})$ (resp. $S_{\mathrm{CK}_{1}}^{1}(\mathbf{X})$ ) the family of Green-tight measures of Kato class (resp. semi-Green-tight measures of extended Kato class) in the sense of [4] with respect to $\mathbf{X}$. Since $S_{\mathrm{CK}_{\infty}}^{1}(\mathbf{X}) \subset S_{\mathrm{NK}_{\infty}}^{1}(\mathbf{X})$ and $S_{\mathrm{CK}_{1}}^{1}(\mathbf{X}) \subset S_{\mathrm{NK}_{1}}^{1}(\mathbf{X})$ (see the remark after Definition 4.2 below), Theorem 1.1 extends [37, Theorem 2.4] (resp. [4, Theorem 5.2] and [5, Theorem 3.4]), in which the case $u=0$ on $E$ and $F=0$ on $E \times E$ (resp. $u=0$ on $E$ ) was treated.

As an application of Theorem 1.1, we prove that the analytic characterization of gaugeability is equivalent to the maximum principle for the generalized Feynman-Kac semigroup under suitable conditions for measures in the framework of resolvent strong Feller symmetric Hunt processes (see Theorems 7.2 and 7.1).

Now let us state the second main result. Let $S_{\mathrm{CS}_{\infty}}^{1}(\mathbf{X})\left(\right.$ resp. $S_{\mathrm{CS}_{1}}^{1}(\mathbf{X})$ ) be the family of conditionally Green-tight measures of Kato class (resp. conditionally semi-Green-tight measures of extended Kato class) in the sense of [4] with respect to $\mathbf{X}, S_{\mathrm{DS}_{0}}^{1}(\mathbf{X})$ (resp. $A_{\mathrm{DS}_{0}}^{1}(\mathbf{X})$ ) the family of conditionally Green-bounded smooth measures in the sense of [4] with respect to $\mathbf{X}$ 
(resp. the family of conditionally Green-bounded jump functions with respect to $\mathbf{X}), B_{\mathrm{DS}_{0}}^{1}(\mathbf{X}):=S_{\mathrm{DS}_{0}}^{1}(\mathbf{X}) \times A_{\mathrm{DS}_{0}}^{1}(\mathbf{X})$ the family of the pairs of elements in $S_{\mathrm{DS}_{0}}^{1}(\mathbf{X})$ and $A_{\mathrm{DS}_{0}}^{1}(\mathbf{X})$, and $B_{\mathrm{CS}_{\infty}}^{1}(\mathbf{X})$ (resp. $B_{\mathrm{CS}_{1}}^{1}(\mathbf{X})$ ) the family of the pairs of conditionally Green-tight measures of Kato class and conditionally Green-tight jump functions (resp. the family of the pair of conditionally semiGreen-tight measures of extended Kato class and conditionally semi-Greentight jump functions) with respect to $\mathbf{X}$ (see Section 6 for the definitions). Let $R^{A}(x, y)$ be the Green kernel of $P_{t}^{A}$ defined by $R^{A}(x, y)=\int_{0}^{\infty} P_{t}^{A}(x, y) \mathrm{d} t$, where $P_{t}^{A}(x, y)$ is the integral kernel of $P_{t}^{A}$.

The second main result of this paper is the following theorem.

Theorem 1.2. Assume $\bar{\mu}_{1}^{*} \in S_{\mathrm{CS}_{1}}^{1}(\mathbf{X}), \quad \mu_{\langle u\rangle} \in S_{\mathrm{CS}_{\infty}}^{1}(\mathbf{X})$ and $\mu_{2}+$ $N\left(F_{2}\right) \mu_{H} \in S_{\mathrm{DS}_{0}}^{1}(\mathbf{X})$. Then the following are equivalent:

(1) The functional (1.1) is semi-conditionally gaugeable, that is, for each $y \in E$

$$
\sup _{x \in E^{y}} \mathbf{E}_{x}^{y}\left[e_{A}\left(\zeta^{y}\right)\right]<\infty .
$$

(2) For each $x \in E, R^{A}(x, y)<\infty$ for $\mathfrak{m}$-a.e. $y \in E^{x}$.

(3) $R^{A}(x, y)<\infty$ for any $(x, y) \in(E \times E) \backslash \mathrm{d}$.

(4) For each $y \in E$, there exists $C>0$ depending only on $y$ such that for any $x \in E^{y} C^{-1} R(x, y) \leq R^{A}(x, y) \leq C R(x, y)$.

(5) $\lambda^{\mathcal{Q}}\left(\bar{\mu}_{1}\right)>0$.

(6) The functional (1.1) is gaugeable, that is, $\sup _{x \in E} \mathbf{E}_{x}\left[e_{A}(\zeta)\right]<\infty$.

Furthermore, if $u=0$ and $\left(\mu_{1}, e^{F_{1}}-1\right) \in B_{\mathrm{CS}_{1}}^{1}(\mathbf{X})$, or $\left(\mu_{1}+\mu_{\langle u\rangle}, F_{1}\right) \in$ $B_{\mathrm{CS}}^{1}(\mathbf{X})$ and there exists $C>0$ such that

$$
C^{-1} R(x, y) \leq R^{U}(x, y) \leq C R(x, y) \quad \text { for all }(x, y) \in(E \times E) \backslash \mathrm{d},
$$

then (1)-(6) are equivalent to (7):

(7) The functional (1.1) is conditionally gaugeable, that is,

$$
\sup _{(x, y) \in(E \times E) \backslash \mathbf{d}} \mathbf{E}_{x}^{y}\left[e_{A}\left(\zeta^{y}\right)\right]<\infty .
$$

Suppose further $\left(\mu_{2}, F_{2}\right) \in B_{\mathrm{DS}_{0}}^{1}(\mathbf{X})$. Moreover, if $u=0$ and $\left(\mu_{1}, e^{F_{1}}-1\right) \in$ $B_{\mathrm{CS}_{1}}^{1}(\mathbf{X})$, or $\left(\mu_{1}+\mu_{\langle u\rangle}, F_{1}\right) \in B_{\mathrm{CS}_{\infty}}^{1}(\mathbf{X})$ with (1.4) holding for some $C>0$, then (1)-(7) are equivalent to (8):

(8) There exists $C>0$ independent of $x, y$ such that

$$
C^{-1} R(x, y) \leq R^{A}(x, y) \leq C R(x, y)
$$

holds for all $(x, y) \in(E \times E) \backslash \mathrm{d}$.

Here $R^{U}(x, y)$ is the Green kernel of the Girsanov transformed process by a multiplicative functional $U_{t}$ defined in (3.5) below. 
REMARK 1.2. Note that the condition $\bar{\mu}_{1}^{*} \in S_{\mathrm{CS}_{1}}^{1}(\mathbf{X})$ is equivalent to $\mu_{1}+$ $N\left(e^{U}\left(e^{F_{1}}-1\right)\right) \mu_{H} \in S_{\mathrm{CS}_{1}}^{1}(\mathbf{X})$ under $\mu_{\langle u\rangle} \in S_{\mathrm{CS}_{\infty}}^{1}(\mathbf{X})$. In [26, Theorem 1.2], we obtain the same conclusion of our Theorem 1.2 under the conditions $\mu_{1} \in$ $S_{\mathrm{CS}_{1}}^{1}(\mathbf{X}), \mu_{\langle u\rangle}+N\left(F_{1}\right) \mu_{H} \in S_{\mathrm{CS}_{\infty}}^{1}(\mathbf{X})$ and $\mu_{2}+N\left(F_{2}\right) \mu_{H} \in S_{\mathrm{DS}_{0}}^{1}(\mathbf{X})$. Our conditions in Theorem 1.2 are milder than them.

If $u=0$ on $E$ and $F_{1}=0$ on $E \times E$, then (1.4) is satisfied with $R^{U}(x, y)=$ $R(x, y)$ for $x, y \in E$ and $\left(\mu_{1}, e^{F_{1}}-1\right) \in B_{\mathrm{CS}_{1}}^{1}(\mathbf{X})$ (resp. $\left.\left(\mu_{1}, F_{1}\right) \in B_{\mathrm{CS}_{\infty}}^{1}(\mathbf{X})\right)$ for $\mu_{1} \in S_{\mathrm{CS}_{1}}^{1}(\mathbf{X})$ (resp. $\mu_{1} \in S_{\mathrm{CS}_{\infty}}^{1}(\mathbf{X})$ ). Thus, Theorem 1.2 covers [4, Theorem 5.3] and [37, Theorem 3.9]. If $u=0$ on $E$ and $F_{1} \in A_{\mathrm{CS}_{\infty}}^{1}(\mathbf{X})$ (see Definition 6.2 below for the definition on $A_{\mathrm{CS}_{\infty}}^{1}(\mathbf{X})$, and Proposition 6.1), then $\left(\mu_{1}, e^{F_{1}}-1\right) \in B_{\mathrm{CS}_{1}}^{1}(\mathbf{X})$ for $\mu_{1} \in S_{\mathrm{CS}_{1}}^{1}(\mathbf{X})$. So Theorem 1.2 under $u=0$ with $\left(\mu_{1}, e^{F_{1}}-1\right) \in B_{\mathrm{CS}_{1}}^{1}(\mathbf{X})$ extends [4, Theorem 3.10] and [5, Theorems 2.1(3) and 3.4]. For general bounded $u$ and $F$ satisfying the conditions in Theorem 1.2, (1.4) is satisfied provided $\mathbf{X}$ is a symmetric diffusion process on $\mathbb{R}^{d}$ with uniform elliptic condition, symmetric stable-like process, or symmetric relativistic stable-like process.

We emphasize that the relaxation of the conditions for measures of the nonlocal part in the creation is significant even in the case $u=0$. This relaxation has not been treated in the previous literature. Theorem 1.1 will be applied to prove the stability of heat kernel estimates for generalized Feynman-Kac semigroup in [25, Theorem 1.1]. The relaxation of the conditions for measures of the non-local part in the creation in Theorem 1.1 is effectively used in the proof of [25, Theorem 1.1(1)].

The basic strategy in obtaining Theorem 1.1 is to decompose the procedure into several steps which is similar to the proof of [26, Theorem 1.1]. However, due to the relaxed conditions for measures, we need to modify the proof of [26, Theorem 1.1]: First, we extend the results on the analytic characterization for local Feynman-Kac transforms developed by [4], [37] in terms of natural Green-tight measures of Kato class and natural semi-Green-tight measures of extended Kato class introduced in Definition 4.2 extending the notion introduced by Chen [4] (Lemmas 4.2, 4.3 and Theorem 4.2). We consider a Girsanov transformed process $\mathbf{U}$ originally introduced by [12] and the analytic characterization for Feynman-Kac functional over the Girsanov transformed process extending [4]. We prove that our analytic characterization implies the gaugeability for (1.1) (Proposition 5.1). Here we use that any $\nu \in S_{\mathrm{NK}_{1}}^{1}\left(\mathbf{X}^{(1)}\right)$ implies $e^{-2 u} \nu \in S_{\mathrm{NK}_{1}}^{1}\left(\mathbf{U}^{(1)}\right)$, where $\mathbf{X}^{(1)}$ (resp. $\left.\mathbf{U}^{(1)}\right)$ is the 1-subprocess of $\mathbf{X}$ (resp. U) (see Lemma 4.1(7) below). This plays a crucial role in our arguments, because $S_{\mathrm{CK}_{1}}^{1}(\mathbf{X})$, the family of semi-Green-tight measures of extended Kato class in the sense of [4], may not be stable under the Girsanov transform even if we consider the 1-subprocess of the transformed process. As a consequence of Proposition 5.1, we have a super gauge theorem for our Feynman-Kac functional (Proposition 5.2), which yields the stability 
of natural (semi-)Green-tightness of (extended) Kato class under the Girsanov transform (Lemma 5.2 and Corollary 5.1). Second, using Corollary 5.1, we prove the assertion of Theorem 1.1

As a consequence of Theorem 1.1, we can obtain the equivalence among gaugeability, semi-conditional gaugeability and subcriticality (the first part of Theorem 1.2). The equivalence between gaugeability and subcriticality has been shown to hold by way of the conditional gaugeability of Feynman-Kac functionals. The first part of Theorem 1.2 shows that there is no need to use the conditional gaugeability to deduce the subcriticality. We also give the equivalence among gaugeability, conditional gaugeability and comparison of Green kernel under (1.4) (the second part of Theorem 1.2). Theorems 1.1 and 1.2 generalize most of the previous known results developed by ChenSong [9], [10], Chen [4], [5] and Takeda [37] for symmetric Markov processes. We remark that the result in [24] together with Theorems 1.1 and 1.2 completely generalizes their results in the framework of symmetric Markov processes.

The remainder of the paper is organized as follows. In Section 2, we explain some basic assumptions on $\mathbf{X}$ and give the definitions of Dynkin and (extended) Kato classes, including a review of several facts from [4], [9], [10], [44]. In Section 3, we study the Girsanov transform induced by the DoléansDade exponential martingale relative to $u$ and $F$, and identify the Dirichlet form associated with the transformed process. In Section 4, we give the definitions of Green-bounded measures, (semi-)Green-tight measures of (extended) Kato class in the sense of [4] and the classes of smooth measures of natural (semi-)Green-tight measures of (extended) Kato class and review several results from [26]. We prove that the family of Green-tight measures of Kato class in the sense of [4] coincides with the family of natural Green-tight measures of Kato class under the doubly Feller property of resolvent (Proposition 4.1). For later use in Section 7, we prove that several preliminary results for gaugeability in [4, Lemmas 2.7, 2.14, Theorems 2.8, 2.15, Corollaries 2.9, 2.16 and Theorems 2.10, 2.11] can be extended under more relaxed conditions for measures when $u=0$. In Section 5, we prove Theorem 1.1. In Section 6, we give the definitions of semi-conditionally Green-bounded measures and semiconditionally (semi-)Green-tight measures of (extended) Kato class and prove Theorem 1.2, the equivalence among gaugeability, semi-conditional (or conditional) gaugeability and subcriticality under the conditional (semi-)Greentightness of the related measures with some condition. In Section 7, we prove that the analytic characterization of gaugeability is equivalent to the maximum principle for the generalized Feynman-Kac functional under mild conditions for measures and the resolvent strong Feller property, in particular, the analytic characterization of gaugeability yields the Liouville property (Theorem 7.1 and Corollary 7.1). Moreover, under the transience of $\mathbf{X}$, the same 
assertion remains valid (Theorem 7.2 and Corollary 7.2). These generalize the recent results by Takeda [42].

We use the following notations: For $a, b \in \mathbb{R}, a \vee b:=\max \{a, b\}, a \wedge b:=$ $\min \{a, b\}$.

\section{Preliminary}

Let $E$ be a locally compact separable metric space and $\mathfrak{m}$ a positive Radon measure on $E$ with full topological support. Let $\partial$ be a point added to $E$ so that $E_{\partial}:=E \cup\{\partial\}$ is the one-point compactification of $E$. The point $\partial$ also serves as the cemetery point for $E$. Let $\mathbf{X}=\left(\Omega, \mathcal{F}_{\infty}, \mathcal{F}_{t}, X_{t}, \mathbf{P}_{x}, x \in E_{\partial}\right)$ be an $\mathfrak{m}$-symmetric transient Hunt process on $E$ with lifetime $\zeta:=\inf \left\{t>0 \mid X_{t}=\right.$ $\partial\}$ and $(\mathcal{E}, \mathcal{F})$ the associated symmetric Dirichlet form which is regular on $L^{2}(E ; \mathfrak{m})$. We say that $(\mathcal{E}, \mathcal{F})$ (or $\left.\mathbf{X}\right)$ is irreducible $((\mathbf{I})$ in abbreviation) if any $\left(T_{t}\right)_{t>0}$-invariant set $B$ satisfies $\mathfrak{m}(B)=0$ or $\mathfrak{m}\left(B^{c}\right)=0$. Here $\left(T_{t}\right)_{t>0}$ is the strongly continuous semigroup on $L^{2}(E ; \mathfrak{m})$ associated with $(\mathcal{E}, \mathcal{F})$. The transition kernel of $\mathbf{X}$ is denoted by $P_{t}(x, \mathrm{~d} y), t>0$. The correspondence between $\mathbf{X}$ and $(\mathcal{E}, \mathcal{F})$ is given by

$$
T_{t} f(x)=\mathbf{E}_{x}\left[f\left(X_{t}\right): t<\zeta\right]:=\int_{E} f(y) P_{t}(x, \mathrm{~d} y) \quad \mathfrak{m} \text {-a.e. } x \in E, t>0 .
$$

(Here and in the sequel, unless mentioned otherwise, we use the convention that a function defined on $E$ takes the value 0 at $\partial$.) The process $\mathbf{X}$ is said to satisfy the absolute continuity condition with respect to $\mathfrak{m}((\mathbf{A C})$ in abbreviation) if for any $x \in E$ and $t>0, \mathfrak{m}(A)=0$ implies $P_{t}(x, A)=0$ for all $A \in \mathcal{B}(E)$. Throughout this paper, $\mathbf{X}$ is assumed to satisfy both $(\mathbf{I})$ and (AC). For $\alpha>0$, there exists an $\alpha$-order resolvent kernel $R_{\alpha}(x, y)$ which is defined for all $x, y \in E$ (see Lemma 4.2.4 in [19]). Since $\alpha \mapsto R_{\alpha}(x, y)$ is decreasing for each $x, y \in E$, we can define the 0 -order resolvent kernel $R(x, y):=R_{0}(x, y):=$ $\lim _{\alpha \rightarrow 0} R_{\alpha}(x, y) . R(x, y)$ is called the Green function of $\mathbf{X}$. For a non-negative Borel measure $\nu$, we write $R_{\alpha} \nu(x):=\int_{E} R_{\alpha}(x, y) \nu(\mathrm{d} y)$ and $R \nu(x):=R_{0} \nu(x)$. Note that $R_{\alpha} f(x)=R_{\alpha}(f \mathfrak{m})(x)$ for any $f \in \mathcal{B}_{+}(E)$ or $f \in \mathcal{B}_{b}(E)$. The space of bounded continuous functions on $E$ will be denoted as $C_{b}(E)$. The process $\mathbf{X}$ is said to satisfy the resolvent strong Feller property ((RSF) in abbreviation) if $R_{\alpha}\left(\mathcal{B}_{b}(E)\right) \subset C_{b}(E)$ for any $\alpha>0$.

Let $S_{1}(\mathbf{X})$ be the family of positive smooth measures in the strict sense [19]. A measure $\nu \in S_{1}(\mathbf{X})$ is said to be in the Dynkin class (resp. Green-bounded) of $\mathbf{X}$ if $\sup _{x \in E} R_{\alpha} \nu(x)<\infty$ for some $\alpha>0$ (resp. $\left.\sup _{x \in E} R \nu(x)<\infty\right)$ ). A measure $\nu \in S_{1}(\mathbf{X})$ is said to be in the Kato class (resp. extended Kato class) with respect to $\mathbf{X}$ if $\lim _{\alpha \rightarrow \infty} \sup _{x \in E} R_{\alpha} \nu(x)=0$ (resp. $<1$ ). A measure $\nu \in S_{1}(\mathbf{X})$ is said to be in the local Kato class if for any compact subset $K$ of $E, \mathbf{1}_{K} \nu$ is of Kato class. Denote by $S_{D}^{1}(\mathbf{X})$ (resp. $S_{D_{0}}^{1}(\mathbf{X})$ ) the family of measures of Dynkin class (resp. Green-bounded), and by $S_{K}^{1}(\mathbf{X})\left(\operatorname{resp} . S_{\mathrm{EK}}^{1}(\mathbf{X}), S_{\mathrm{LK}}^{1}(\mathbf{X})\right)$ the family of measures of Kato class (resp. extended Kato class, local Kato class). 
Clearly, $S_{K}^{1}(\mathbf{X}) \subset S_{\mathrm{EK}}^{1}(\mathbf{X}) \subset S_{D}^{1}(\mathbf{X}), S_{K}^{1}(\mathbf{X}) \subset S_{\mathrm{LK}}^{1}(\mathbf{X})$ and $S_{D_{0}}^{1}(\mathbf{X}) \subset S_{D}^{1}(\mathbf{X})$. In view of Theorem 3.1 in [36], any $\nu \in S_{D}^{1}(\mathbf{X})$, in particular any $\nu \in S_{D_{0}}^{1}(\mathbf{X})$, is a Radon measure on $E$, because of the regularity of the Dirichlet form. We say that a positive continuous additive functional (PCAF in abbreviation) in the strict sense $A^{\nu}$ of $\mathbf{X}$ and a positive measure $\nu \in S_{1}(\mathbf{X})$ are in the Revuz correspondence if they satisfy for any $t>0, f \in \mathcal{B}_{+}(E)$,

$$
\int_{E} f(x) \nu(\mathrm{d} x)=\uparrow \lim _{t \downarrow 0} \frac{1}{t} \mathbf{E}_{\mathfrak{m}}\left[\int_{0}^{t} f\left(X_{s}\right) \mathrm{d} A_{s}^{\nu}\right] .
$$

It is known that the family of equivalence classes of the set of PCAFs in the strict sense and the family of positive measures belonging to $S_{1}(\mathbf{X})$ are in one to one correspondence under the Revuz correspondence ([19, Theorem 5.1.4]).

An increasing sequence $\left\{F_{k}\right\}$ of closed sets is said to be an $\mathcal{E}$-nest if

$$
\mathbf{P}_{x}\left(\lim _{k \rightarrow \infty} \sigma_{E \backslash F_{k}} \geq \zeta\right)=1 \quad \text { m-a.e. } x \in E .
$$

A function $f$ on $E$ is said to be $\mathcal{E}$-quasi-continuous if there exists an $E$-nest such that $\left.f\right|_{F_{k}}$ is continuous for each $k \in \mathbb{N}$. Let $\left(\mathcal{E}, \mathcal{F}_{e}\right)$ be the extended Dirichlet space of $(\mathcal{E}, \mathcal{F})$ (see [19] for the definition). Any element $f \in \mathcal{F}_{e}$ admits an $\mathcal{E}$-quasi-continuous $\mathfrak{m}$-version $\tilde{f}$ (see [19]). Throughout this paper, we always take an $\mathcal{E}$-quasi-continuous $\mathfrak{m}$-version of the element of $\mathcal{F}_{e}$, that is, we omit tilde from $\tilde{f}$ for $f \in \mathcal{F}_{e}$. Let $\left(N(x, \mathrm{~d} y), H_{t}\right)$ be a Lévy system for $\mathbf{X}$, that is, $N(x, \mathrm{~d} y)$ is a kernel on $\left(E_{\partial}, \mathcal{B}\left(E_{\partial}\right)\right)$ and $H_{t}$ is a PCAF with bounded 1-potential such that for any nonnegative Borel function $\phi$ on $E_{\partial} \times$ $E_{\partial}$ vanishing on the diagonal and any $x \in E_{\partial}$, nonnegative Borel function $g$ on $\left[0, \infty\left[\right.\right.$ and $\left(\mathcal{F}_{t}\right)$-stopping time $T$,

$$
\mathbf{E}_{x}\left[\sum_{s \leq T} g(s) \phi\left(X_{s-}, X_{s}\right)\right]=\mathbf{E}_{x}\left[\int_{0}^{T} \int_{E_{\partial}} g(s) \phi\left(X_{s}, y\right) N\left(X_{s}, \mathrm{~d} y\right) \mathrm{d} H_{s}\right]
$$

(see $[7$, A.3.33]). To simplify notation, we will write

$$
N \phi(x):=\int_{E_{\partial}} \phi(x, y) N(x, \mathrm{~d} y) .
$$

Let $\mu_{H}$ be the Revuz measure of the PCAF $H$. Then the jumping measure $J$ and the killing measure $\kappa$ of $\mathbf{X}$ are given by

$$
J(\mathrm{~d} x \mathrm{~d} y)=\frac{1}{2} N(x, \mathrm{~d} y) \mu_{H}(\mathrm{~d} x) \quad \text { and } \quad \kappa(\mathrm{d} x)=N(x,\{\partial\}) \mu_{H}(\mathrm{~d} x) .
$$

These measures feature in the Beurling-Deny decomposition of $\mathcal{E}$ :

$$
\begin{aligned}
\mathcal{E}(f, g)= & \mathcal{E}^{c}(f, g)+\int_{(E \times E) \backslash \text { diag }}(f(x)-f(y))(g(x)-g(y)) J(\mathrm{~d} x \mathrm{~d} y) \\
& +\int_{E} f(x) g(x) \kappa(\mathrm{d} x)
\end{aligned}
$$

for $f, g \in \mathcal{F}_{e}$. Here $\mathcal{E}^{c}$ is the strongly local part of $\mathcal{E}$. 
A function $f$ on $E$ is said to be locally in $\mathcal{F}$ in the broad sense if there exists a nest $\left\{G_{n}\right\}$ of finely open (nearly) Borel sets and a sequence $\left\{f_{n}\right\}$ of elements in $\mathcal{F}$ such that $f=f_{n}$ m-a.e. on $G_{n}$. Let $\dot{\mathcal{F}}_{\text {loc }}$ be the family of functions on $E$ locally in $\mathcal{F}$ in the broad sense. It is known that any $f \in \dot{\mathcal{F}}_{\text {loc }}$ admits $\mathcal{E}$-quasi-continuous $\mathfrak{m}$-version.

An increasing sequence $\left\{F_{k}\right\}$ of closed sets is said to be a strict $\mathcal{E}$-nest if $\mathbf{P}_{x}\left(\lim _{k \rightarrow \infty} \sigma_{E \backslash F_{k}}=\infty\right)=1$ m-a.e. $x \in E$. A function $f$ defined on $E_{\partial}$ is said to be strictly $\mathcal{E}$-quasi-continuous if there exists a strict $\mathcal{E}$-nest $\left\{F_{k}\right\}$ of closed sets such that $\left.f\right|_{F_{k} \cup\{\partial\}}$ is continuous for each $k \in \mathbb{N}$. Denote by $Q C\left(E_{\partial}\right)$ the totality of strictly $\mathcal{E}$-quasi-continuous functions on $E_{\partial}$. To the end of this section, we consider a bounded finely continuous (nearly) Borel function $u \in \dot{\mathcal{F}}_{\text {loc }} \cap Q C\left(E_{\partial}\right)$ satisfying $\mu_{\langle u\rangle} \in S_{D}^{1}(\mathbf{X})$. In [27, Theorem 6.2(2)], we proved that the additive functional $u\left(X_{t}\right)-u\left(X_{0}\right)$ admits the following strict decomposition:

$$
u\left(X_{t}\right)-u\left(X_{0}\right)=M_{t}^{u}+N_{t}^{u}, \quad t \in\left[0, \infty\left[\mathbf{P}_{x} \text {-a.s. for all } x \in E,\right.\right.
$$

where $M^{u}$ is a square integrable martingale additive functional in the strict sense, and $N^{u}$ is a continuous additive functional (CAF in abbreviation) in the strict sense which is locally of zero energy. $M^{u}$ can be decomposed as

$$
M_{t}^{u}=M_{t}^{u, c}+M_{t}^{u, j}+M_{t}^{u, \kappa}
$$

where $M_{t}^{u, j}, M_{t}^{u, \kappa}$ and $M_{t}^{u, c}$ are the jumping, killing and continuous part of $M^{u}$, respectively. Those are defined $\mathbf{P}_{x}$-a.s. for all $x \in E$ by [27, Theorem $6.2(2)]$. The strict decompositions $(2.2)$ and $(2.3)$ on $[0, \infty[$ guarantee the extension of the supermartingale multiplicative functional $Y_{t}$ on $\llbracket 0, \zeta \llbracket$ up to $\left[0, \infty\left[\right.\right.$ (see Proposition 3.1 below). Let $\mu_{\langle u\rangle}, \mu_{\langle u\rangle}^{c}, \mu_{\langle u\rangle}^{j}$ and $\mu_{\langle u\rangle}^{\kappa}$ be the smooth Revuz measures in the strict sense associated with the quadratic variational processes (or the sharp bracket PCAFs in the strict sense) $\left\langle M^{u}\right\rangle,\left\langle M^{u, c}\right\rangle$, $\left\langle M^{u, j}\right\rangle$ and $\left\langle M^{u, \kappa}\right\rangle$, respectively. Then

$$
\mu_{\langle u\rangle}(\mathrm{d} x)=\mu_{\langle u\rangle}^{c}(\mathrm{~d} x)+\mu_{\langle u\rangle}^{j}(\mathrm{~d} x)+\mu_{\langle u\rangle}^{\kappa}(\mathrm{d} x) .
$$

Note that $\mathcal{E}(f, f)=\frac{1}{2} \nu_{\langle f\rangle}(E)$ with $\nu_{\langle f\rangle}:=\mu_{\langle f\rangle}^{c}+\mu_{\langle f\rangle}^{j}+2 \mu_{\langle f\rangle}^{\kappa}$ provided $f \in \mathcal{F}_{e}$.

Let $\nu_{1}$ (resp. $\nu_{2}$ ) be a positive (resp. signed) Radon measure on $E$. Let $(\mathcal{A}, \mathcal{D}(\mathcal{A}))$ be a lower bounded closed symmetric form on $L^{2}(E ; \mathfrak{m})$ having core $\mathcal{C}$ satisfying $(\mathcal{C}, 1),(\mathcal{C}, 2)$ in $[19]$ such that the perturbed form $\left(\mathcal{A}^{\nu_{2}}, \mathcal{C}\right)$ defined by $\mathcal{A}^{\nu_{2}}(f, g)=\mathcal{A}(f, g)+\int_{E} f g \mathrm{~d} \nu_{2}, f, g \in \mathcal{C}$ is also lower bounded. We set

$$
\lambda\left(\nu_{2}, \nu_{1}\right):=\inf \left\{\mathcal{A}^{\nu_{2}}(f, f) \mid f \in \mathcal{C}, \int_{E} f^{2} \mathrm{~d} \nu_{1}=1\right\} .
$$

Then we have the following lemma due to Takeda-Uemura [44]. 
Lemma 2.1 (cf. Lemma 3.1 in [44]). Let $\nu$ be another positive Radon measure on $E$. Then $\lambda\left(\nu_{2}+\nu, \nu_{1}+\nu\right)>1$ implies $\lambda\left(\nu_{2}, \nu_{1}\right)>1$. Suppose that $\lambda\left(\nu_{2}, \nu\right)>0$. Then the converse assertion holds.

REMARK 2.1. Lemma 3.1 in [44] is stated in the framework of rotationally symmetric $\alpha$-stable processes, and $\nu_{2}=\mu_{-}$and $\nu_{1}=\mu_{+}$are the negative and positive parts in the Jordan-Hahn decomposition of the signed smooth Radon measure $\mu$. However, its proof remains valid in this generality.

\section{Girsanov transforms}

Let $F$ be a bounded symmetric function on $E \times E$, which is extended to a function $F$ defined on $E_{\partial} \times E_{\partial}$ by setting $F(x, \partial)=F(\partial, x)=F(x, x)=0$ for $x \in E_{\partial}$ (actually there is no need to define the value $F(\partial, y)$ for $y \in E$ ). Set $F_{+}:=F \vee 0$ and $F_{-}:=(-F) \vee 0$. We say that $F:=F_{+}-F_{-}$is in the class $J_{1}(\mathbf{X})\left(\right.$ resp. $J_{D}^{1}(\mathbf{X})$ and $\left.J_{D_{0}}^{1}(\mathbf{X})\right)$ if $N(|F|) \mu_{H}$ belongs to $S_{1}(\mathbf{X})\left(\right.$ resp. $S_{D}^{1}(\mathbf{X})$ and $\left.S_{D_{0}}^{1}(\mathbf{X})\right)$, where $|F|=F_{+}+F_{-}$. Any such an $F$ has an expression of the form $F=F_{1}-F_{2}$, where each $F_{i}(i=1,2)$ is a symmetric nonnegative bounded function on $E_{\partial} \times E_{\partial}$ vanishing on the diagonal set of $E_{\partial} \times E_{\partial}$. Indeed, for any nonnegative symmetric bounded $\phi$ having the same property as $F, F_{1}:=F_{+}+\phi$ and $F_{2}:=F_{-}+\phi$ have these properties. Note that if $F_{1}+F_{2} \in J_{1}(\mathbf{X})\left(\right.$ resp. $J_{D}^{1}(\mathbf{X})$ and $\left.J_{D_{0}}^{1}(\mathbf{X})\right)$, then $F \in J_{1}(\mathbf{X})$ (resp. $J_{D}^{1}(\mathbf{X})$ and $J_{D_{0}}^{1}(\mathbf{X})$ ). In this case, the following $A^{F}$ can be defined as an additive functional in the strict sense:

$$
A_{t}^{F}=A_{t}^{F_{1}}-A_{t}^{F_{2}}, \quad A_{t}^{F_{i}}:=\sum_{0<s \leq t} F_{i}\left(X_{s-}, X_{s}\right) \quad(i=1,2) .
$$

Note that $A_{t}^{F}=\sum_{0<s \leq t} \mathbf{1}_{\{s<\zeta\}} F\left(X_{s-}, X_{s}\right)$, since $F(x, \partial)=0$ for $x \in E_{\partial}$. For a bounded finely continuous (nearly) Borel function $u \in \mathcal{F}_{\text {loc }} \cap Q C\left(E_{\partial}\right)$ satisfying $\mu_{\langle u\rangle} \in S_{1}(\mathbf{X})$ and such $F \in J_{1}(\mathbf{X})$, we set

$$
F^{u}(x, y):=F(x, y)+\{-u(y)-(-u(x))\}=F(x, y)+u(x)-u(y)
$$

and $G^{u}=e^{F^{u}}-1$ with identifying $F^{0}=F$ and $G^{0}=G:=e^{F}-1$. Since

$$
\left|F^{u}(x, y)\right|^{2} \leq 2\|F\|_{\infty}|F(x, y)|+2(u(x)-u(y))^{2},
$$

one can see that the relation $N\left(\left|F^{u}\right|^{2}\right) \mu_{H} \leq 2\|F\|_{\infty} N(|F|) \mu_{H}+\mu_{\langle u\rangle}$ implies $\left(F^{u}\right)^{2} \in J_{1}(\mathbf{X})$. So there exists a purely discontinuous locally square integrable local martingale additive functional $M^{F^{u}}$ on $\llbracket 0, \zeta \llbracket$ such that $\Delta M_{t}^{F^{u}}=$ $F^{u}\left(X_{t-}, X_{t}\right), t \in\left[0, \zeta\left[\mathbf{P}_{x^{-}}\right.\right.$a.s. for all $x \in E$ (see the proof of Lemma 3.2(i) in [13]). Moreover, $M_{t}^{F^{u}}$ is given by

$$
M_{t}^{F^{u}}=M_{t}^{F}+M_{t}^{-u, j}+M_{t}^{-u, \kappa},
$$


where

$$
M_{t}^{F}=\sum_{0<s \leq t} F\left(X_{s-}, X_{s}\right)-\int_{0}^{t} N(F)\left(X_{s}\right) \mathrm{d} H_{s}, \quad t<\zeta .
$$

On the other hand, since $\left|e^{z}-z-1\right| \leq \frac{1}{2} e^{|z|}|z|^{2}$, the relations

$$
\left|G^{u}(x, y)-F^{u}(x, y)\right| \leq \frac{1}{2} e^{\left\|F^{u}\right\|_{\infty}}\left|F^{u}(x, y)\right|^{2}
$$

and

$$
\begin{aligned}
\left|G^{u}(x, y)\right|^{2} & \leq\left(\frac{\left\|F^{u}\right\|_{\infty} e^{\left|F^{u}(x, y)\right|}}{2}\left|F^{u}(x, y)\right|+\left|F^{u}(x, y)\right|\right)^{2} \\
& \leq\left(\frac{\left\|F^{u}\right\|_{\infty} e^{\left\|F^{u}\right\|_{\infty}}}{2}+1\right)^{2}\left|F^{u}(x, y)\right|^{2}
\end{aligned}
$$

imply $G^{u}-F^{u} \in J_{1}(\mathbf{X})$ and $\left(G^{u}\right)^{2} \in J_{1}(\mathbf{X})$ respectively, because $\left(F^{u}\right)^{2} \in$ $J_{1}(\mathbf{X})$. Similarly, we have $G-F \in J_{1}(\mathbf{X})$ and $G^{2} \in J_{1}(\mathbf{X})$. Therefore, there also exists a purely discontinuous locally square integrable local martingale additive functional $M^{G^{u}}$ such that $\Delta M_{t}^{G^{u}}=G^{u}\left(X_{t-}, X_{t}\right), t \in\left[0, \zeta\left[\mathbf{P}_{x^{-}}\right.\right.$a.s. for all $x \in E . M_{t}^{G^{u}}$ is given by

$$
\begin{aligned}
M_{t}^{G^{u}}= & M_{t}^{F^{u}}+\sum_{0<s \leq t}\left(G^{u}-F^{u}\right)\left(X_{s-}, X_{s}\right) \\
& -\int_{0}^{t} N\left(G^{u}-F^{u}\right)\left(X_{s}\right) \mathrm{d} H_{s}, \quad t<\zeta .
\end{aligned}
$$

Put $M_{t}:=M_{t}^{G^{u}}+M_{t}^{-u, c}$ and let

$$
Y_{t}:=\operatorname{Exp}(M)_{t}, \quad t<\zeta
$$

be the solution of the SDE

$$
Y_{t}=1+\int_{0}^{t} Y_{s-} \mathrm{d} M_{s}, \quad t<\zeta, \mathbf{P}_{x} \text {-a.s. }
$$

Note that $Y_{t}$ is positive and a local martingale on $\llbracket 0, \zeta \llbracket$. Therefore it is a supermartingale on $\llbracket 0, \zeta \llbracket$. In particular, $Y_{t} \mathbf{1}_{\{t<\zeta\}}$ is a supermartingale with $\mathbf{E}_{x}\left[Y_{t} \mathbf{1}_{\{t<\zeta\}}\right] \leq 1$ for all $x \in E$. It is inconvenient to treat additive functionals on $\llbracket 0, \zeta \llbracket$ for our purpose. We need a sufficient condition for $Y_{t}$ to be defined for all $t \in[0, \infty[$.

Proposition 3.1 (Proposition 3.1 in [26]). Assume that $F \in J_{D}^{1}(\mathbf{X})$ and $u \in \dot{\mathcal{F}}_{\text {loc }} \cap Q C\left(E_{\partial}\right)$ is a bounded finely continuous (nearly) Borel function satisfying $\mu_{\langle u\rangle} \in S_{D}^{1}(\mathbf{X})$. Then $Y_{t}$ can be regarded to be a supermartingale multiplicative functional defined for all $t \in\left[0, \infty\left[\mathbf{P}_{x}\right.\right.$-a.s. for all $x \in E$. 
Let $\mathbf{Y}=\left(\Omega, \widetilde{\mathcal{F}}_{\infty}, \widetilde{\mathcal{F}}_{t}, \widetilde{X}_{t}, \mathbf{P}_{x}^{Y}, \zeta\right)$ be the transformed process of $\mathbf{X}$ by $Y_{t}$. The transition semigroup $\left\{P_{t}^{Y}\right\}_{t \geq 0}$ of $\mathbf{Y}$ is defined by

$$
P_{t}^{Y} f(x):=\mathbf{E}_{x}^{Y}\left[f\left(\widetilde{X}_{t}\right)\right]=\mathbf{E}_{x}\left[Y_{t} f\left(X_{t}\right)\right] .
$$

Theorem 3.1 (Theorem 3.1 in [26]). Assume that $F \in J_{1}(\mathbf{X})$ and $u \in$ $\dot{\mathcal{F}}_{\text {loc }} \cap Q C\left(E_{\partial}\right)$ is a bounded finely continuous (nearly) Borel function satisfying $\mu_{\langle u\rangle} \in S_{1}(\mathbf{X})$. Then the following hold.

(1) $Y_{t}$ can be represented as follows:

$$
\begin{aligned}
Y_{t}= & \exp \left(M_{t}^{F^{u}}+M_{t}^{-u, c}-\int_{0}^{t} N\left(G^{u}-F^{u}\right)\left(X_{s}\right) \mathrm{d} H_{s}\right. \\
& \left.-\frac{1}{2}\left\langle M^{u, c}\right\rangle_{t}\right), \quad t<\zeta .
\end{aligned}
$$

(2) $\mathbf{Y}$ is an $e^{-2 u} \mathfrak{m}$-symmetric Hunt process on $E$.

When $F_{2}=0$, we write the Girsanov transformed process $\mathbf{Y}^{1}$ by $Y_{t}^{1}$ instead of $\mathbf{Y}$. When $F_{1}=F_{2}$, we write the Girsanov transformed process $\mathbf{U}$ by

$$
U_{t}:=\operatorname{Exp}\left(M^{e^{U}-1}+M^{-u, c}\right)_{t}
$$

instead of $\mathbf{Y}$. Let $\mathbf{Z}=\left(\Omega, \widetilde{\mathcal{F}}_{\infty}, \widetilde{\mathcal{F}}_{t}, \widetilde{X}_{t}, \mathbf{P}_{x}^{Z}, \zeta\right)$ be the transformed process of $\mathbf{X}$ by the supermartingale multiplicative functional

$$
Z_{t}:=Y_{t}^{1} \exp \left(-A_{t}^{F_{2}}\right)
$$

and $\left(\mathcal{E}^{Z}, \mathcal{F}^{Z}\right)$ the Dirichlet form on $L^{2}\left(E ; e^{-2 u} \mathfrak{m}\right)$ associated with $\mathbf{Z}$. In a similar way as in the proof of Theorem 3.1(1), we see that

$$
Z_{t}=Y_{t} \exp \left(-\int_{0}^{t} N\left(G_{1}^{u}-G^{u}\right)\left(X_{s}\right) \mathrm{d} H_{s}\right), \quad t \in[0, \infty[.
$$

Theorem 3.2 (Theorem 3.2 in [26]). Suppose $F \in J_{1}(\mathbf{X})$ and $u$ is a bounded strictly $\mathcal{E}$-quasi-continuous function defined on $E_{\partial}$ such that $u \in \dot{\mathcal{F}}_{\text {loc }}$ admits Fukushima's decomposition holding up to infinity under $\mathbf{P}_{x}$-a.s. for q.e. starting point $x \in E$. Let $\left(\mathcal{E}^{Y}, \mathcal{F}^{Y}\right)$ be the Dirichlet form of $\mathbf{Y}$ on $L^{2}\left(E ; e^{-2 u} \mathfrak{m}\right)$. Then $\mathcal{F}=\mathcal{F}^{Y}$ and

$$
\begin{aligned}
\mathcal{E}^{Y}(f, f)= & \frac{1}{2} \int_{E} e^{-2 u(x)} \mu_{\langle f\rangle}^{c}(\mathrm{~d} x) \\
& +\int_{(E \times E) \backslash \mathrm{diag}}(f(x)-f(y))^{2} e^{F(x, y)-u(x)-u(y)} J(\mathrm{~d} x \mathrm{~d} y) \\
& +e^{-u(\partial)} \int_{E} f(x)^{2} e^{-u(x)} \kappa(\mathrm{d} x)
\end{aligned}
$$

for any $f \in \mathcal{F}^{Y}$. In particular, we have $\mathcal{F}_{e}^{Y}=\mathcal{F}_{e}$ and there is $C=C(u, F)>0$ such that $C^{-1} \mu_{\langle f\rangle}^{c} \leq \mu_{\langle f\rangle}^{Y, c} \leq C \mu_{\langle f\rangle}^{c}$ for all $f \in \mathcal{F}, C^{-1} J \leq J^{Y} \leq C J$ on 
$(E \times E) \backslash \operatorname{diag}$ and $C^{-1} \kappa \leq \kappa^{Y} \leq C \kappa$, hence $\left(\mathcal{E}^{Y}, \mathcal{F}^{Y}\right)$ is a transient Dirichlet form on $L^{2}\left(E ; e^{-2 u} \mathfrak{m}\right)$. Here $\mu_{\langle f\rangle}^{Y, c}(\mathrm{~d} x):=e^{-2 u(x)} \mu_{\langle f\rangle}^{c}(\mathrm{~d} x), J^{Y}(\mathrm{~d} x \mathrm{~d} y):=$ $e^{F(x, y)-u(x)-u(y)} J(\mathrm{~d} x \mathrm{~d} y)$ and $\kappa^{Y}(\mathrm{~d} x):=e^{-u(x)-u(\partial)} \kappa(\mathrm{d} x)$. Moreover, if $\kappa=0$, then the conclusion remains valid under that Fukushima's decomposition holds up to $\zeta$ under $\mathbf{P}_{x}$ for q.e. $x \in E$.

We need the coincidence of the fine topologies of $\mathbf{Y}$ and $\mathbf{X}$ :

LEMMA 3.1. Let $u \in \mathcal{F}_{\text {loc }} \cap Q C\left(E_{\partial}\right)$ be a bounded finely continuous (nearly) Borel function satisfying $\mu_{\langle u\rangle} \in S_{D}^{1}(\mathbf{X})$ and assume $F \in J_{D}^{1}(\mathbf{X})$. Then the fine topology of $\mathbf{Y}$ equals that of $\mathbf{X}$.

Proof. By Proposition 3.1, $\left(Y_{t}\right)_{t \in[0,+\infty[}$ is a $\mathbf{P}_{x}$-supermartingale multiplicative functional on $[0,+\infty$ [ for all $x \in E$. It suffices to prove that the family of all finely open Borel sets for $\mathbf{Y}$ coincides with that for $\mathbf{X}$, because any $\alpha$-excessive functions of $\mathbf{X}$ (also of $\mathbf{Y}$ ) is Borel measurable under (AC). Let $f$ be a finely continuous Borel function with respect to $\mathbf{X}$. First, we prove the fine continuity of $f$ with respect to $\mathbf{Y}$. It is known that the fine continuity of $f$ is equivalent to the right continuity of $\left[0,+\infty\left[\ni s \mapsto f\left(X_{s}\right)\right.\right.$ under $\mathbf{P}_{x}$ for all $x \in E$ and the right continuity of $s \mapsto f\left(X_{s}\right)$ at $s=0$ under $\mathbf{P}_{x}$ implies the fine continuity of $f$ with respect to $\mathbf{X}$ (see $[3$, the proof of (4.8) Theorem], [19, Theorem A.2.7]). The event $A:=\left\{\omega \in \Omega \mid s \mapsto f\left(X_{s}\right)\right.$ is right continuous at $s=0\}$ belongs to $\mathcal{F}_{t}^{0}:=\sigma\left\{X_{s}: s \leq t\right\} \subset \mathcal{F}_{t}$ for any $t>0$. So we have $\mathbf{P}_{x}^{Y}\left(A^{c}\right)=\mathbf{P}_{x}^{Y}\left(A^{c} \cap\{t \geq \zeta\}\right)+\mathbf{P}_{x}^{Y}\left(A^{c} \cap\{t<\zeta\}\right) \leq \mathbf{P}_{x}^{Y}(t \geq \zeta)+\mathbf{E}_{x}\left[Y_{t} \mathbf{1}_{A^{c}}\right.$ : $t<\zeta]=\mathbf{P}_{x}^{Y}(t \geq \zeta) \rightarrow 0$ as $t \rightarrow 0$. Therefore, $f$ is finely continuous with respect to $\mathbf{Y}$. Conversely suppose that $f$ is a finely continuous Borel function with respect to $\mathbf{Y}$. Then the event $A:=\left\{\omega \in \Omega \mid s \mapsto f\left(X_{s}\right)\right.$ is right continuous at $s=0\}$ also belongs to $\mathcal{F}_{t}^{Y}$ for $t>0$ and it satisfies $\mathbf{P}_{x}^{Y}\left(A^{c}\right)=0$ for all $x \in E$. Then $\mathbf{E}_{x}\left[Y_{t} \mathbf{1}_{A^{c}}: t<\zeta\right]=\mathbf{P}_{x}^{Y}\left(A^{c} \cap\{t<\zeta\}\right)=0$ for all $t>0$ and $x \in E$. Thus $\mathbf{P}_{x}\left(A^{c} \cap\{t<\zeta\}\right)=0$ for all $t>0$ and $x \in E$, because $\mathbf{P}_{x}\left(Y_{t}>0\right.$ for $t \in\left[0, \zeta[)=1\right.$ for all $x \in E$. Letting $t \rightarrow 0$, we have $\mathbf{P}_{x}(A)=1$ for all $x \in E$. Therefore, we obtain the fine continuity of $f$ with respect to $\mathbf{X}$.

\section{4. (Semi-) Green-tight measures of (extended) Kato classes and gaugeability}

Let $\mu=\mu_{1}-\mu_{2}$ be a signed smooth measure in the strict sense whose associated CAF of $\mathbf{X}$ is $A^{\mu}:=A^{\mu_{1}}-A^{\mu_{2}}$. Here $A^{\mu_{1}}$ and $A^{\mu_{2}}$ are the PCAFs of $\mathbf{X}$ with Revuz measures $\mu_{1} \in S_{1}(\mathbf{X})$ and $\mu_{2} \in S_{1}(\mathbf{X})$, respectively. Now we start with the notion of Green-tight measures of (extended) Kato class in the strict sense given in [4, Definition 2.2].

Definition 4.1 (Green-tight Kato class measures). Let $\nu \in S_{1}(\mathbf{X})$ and $\alpha \geq 0$. 
(1) $\nu$ is said to be an $\alpha$-order Green-tight measure of Kato class with respect to $\mathbf{X}$ if $\nu \in S_{K}^{1}(\mathbf{X})$ and for any $\varepsilon>0$ there exists a compact subset $K=K(\varepsilon)$ of $E$ such that

$$
\sup _{x \in E} R_{\alpha}\left(\mathbf{1}_{K^{c}} \nu\right)(x)=\sup _{x \in E} \int_{K^{c}} R_{\alpha}(x, y) \nu(\mathrm{d} y)<\varepsilon .
$$

(2) $\nu$ is said to be an $\alpha$-order semi-Green-tight measure of extended Kato class with respect to $\mathbf{X}$ if $\nu \in S_{\mathrm{EK}}^{1}(\mathbf{X})$ and there exists a compact subset $K$ of $E$ such that

$$
\sup _{x \in E} R_{\alpha}\left(\mathbf{1}_{K^{c}} \nu\right)(x)=\sup _{x \in E} \int_{K^{c}} R_{\alpha}(x, y) \nu(\mathrm{d} y)<1 .
$$

(3) $\nu$ is said to be an $\alpha$-order Green-tight measure of Kato class with respect to $\mathbf{X}$ in the sense of Chen if for any $\varepsilon>0$ there exist a Borel subset $K=K(\varepsilon)$ of $E$ with $\nu(K)<\infty$ and a constant $\delta>0$ such that for all $\nu$-measurable set $B \subset K$ with $\nu(B)<\delta$,

$$
\sup _{x \in E} R_{\alpha}\left(\mathbf{1}_{B \cup K^{c}} \nu\right)(x)=\sup _{x \in E} \int_{B \cup K^{c}} R_{\alpha}(x, y) \nu(\mathrm{d} y)<\varepsilon .
$$

(4) $\nu$ is said to be an $\alpha$-order semi-Green-tight measure of extended Kato class with respect to $\mathbf{X}$ in the sense of Chen if there exist a Borel subset $K$ of $E$ with $\nu(K)<\infty$ and a constant $\delta>0$ such that for all $\nu$-measurable set $B \subset K$ with $\nu(B)<\delta$,

$$
\sup _{x \in E} R_{\alpha}\left(\mathbf{1}_{B \cup K^{c}} \nu\right)(x)=\sup _{x \in E} \int_{B \cup K^{c}} R_{\alpha}(x, y) \nu(\mathrm{d} y)<1 .
$$

In view of the resolvent equation, for positive $\alpha$, the $\alpha$-order Greentightness of Kato class is independent of the choice of $\alpha>0$. Let us denote by $S_{K_{\infty}^{+}}^{1}(\mathbf{X})$ (resp. $S_{\mathrm{CK}_{\infty}^{+}}^{1}(\mathbf{X})$ ) the family of positive order Green-tight measures of Kato class (resp. the family of positive order Green-tight measures of Kato class in the sense of Chen) with respect to $\mathbf{X}$. The class $S_{K_{\infty}}^{1}(\mathbf{X})$ (resp. $S_{K_{1}}^{1}(\mathbf{X})$ ) is then used to denote the family of 0-order Green-tight measures of Kato class (resp. 0-order semi-Green-tight measures of extended Kato class), and the class $S_{\mathrm{CK}_{\infty}}^{1}(\mathbf{X})$ (resp. $\left.S_{\mathrm{CK}_{1}}^{1}(\mathbf{X})\right)$ is then used to denote the family of 0-order Green-tight measures of Kato class in the sense of Chen (resp. the family of 0 -order semi-Green-tight measures of extended Kato class in the sense of Chen) with respect to $\mathbf{X}$. Note that since a Green kernel is invariant under time change by the PCAF associated to a non-negative smooth measure with full quasi support, the definitions of $S_{\mathrm{CK}_{\infty}}^{1}(\mathbf{X})$ and $S_{\mathrm{CK}_{1}}^{1}(\mathbf{X})$ are invariant under such time change in contrast to the Kato class $S_{K}^{1}(\mathbf{X})$. It is proved in [4] that $S_{\mathrm{CK}_{\infty}}^{1}(\mathbf{X}) \subset S_{\mathrm{CK}_{1}}^{1}(\mathbf{X}) \subset S_{D_{0}}^{1}(\mathbf{X}) \cap S_{\mathrm{EK}}^{1}(\mathbf{X}), S_{\mathrm{CK}_{\infty}^{+}}^{1}(\mathbf{X}) \subset S_{K}^{1}(\mathbf{X})$ and $S_{\mathrm{CK}}^{1}(\mathbf{X}) \subset S_{K}^{1}(\mathbf{X})$. Since any measure $\nu$ in Definition 4.1 belongs to $S_{D_{0}}^{1}(\mathbf{X})$, it is a Radon measure. The Borel set $K$ in Definition 4.1(3), (4) can be taken 
to be a closed set or an open set (see [4, remark after Definition 2.2]). Moreover, such closed set $K$ can be taken to be compact, in particular, we always have $S_{\mathrm{CK}_{\infty}^{+}}^{1}(\mathbf{X}) \subset S_{K_{\infty}^{+}}^{1}(\mathbf{X}), S_{\mathrm{CK}_{\infty}}^{1}(\mathbf{X}) \subset S_{K_{\infty}}^{1}(\mathbf{X})$ and $S_{\mathrm{CK}_{1}}^{1}(\mathbf{X}) \subset S_{K_{1}}^{1}(\mathbf{X})$. Indeed, take $\nu \in S_{\mathrm{CK}_{\infty}^{+}}^{1}(\mathbf{X}), \varepsilon>0$, and a closed set $K$ such that (4.1) holds. Then there exists a compact subset $C \subset K$ with $\nu(K \backslash C)<\delta$ for a given $\delta>0$, because $\nu \in S_{D_{0}}^{1}(\mathbf{X})$ is a Radon measure. Since $\nu \in S_{\mathrm{CK}_{\infty}^{+}}^{1}(\mathbf{X})$, we have $\sup _{x \in E} R_{\alpha} \mathbf{1}_{C^{c}} \nu(x)=\sup _{x \in E} R_{\alpha} \mathbf{1}_{K^{c} \cup(K \backslash C)} \nu(x)<\varepsilon$.

Let $\nu \in S_{1}(\mathbf{X})$ and denote by $A_{t}^{\nu}$ PCAF in the strict sense associated to $\nu$ in Revuz correspondence. Denote by $\mathrm{S}_{o}^{\nu}$ the support of $A^{\nu}$ defined by $\mathrm{S}_{o}^{\nu}:=\left\{x \in E \mid \mathbf{P}_{x}(R=0)=1\right\}$, where $R(\omega):=\inf \left\{t>0 \mid A_{t}^{\nu}(\omega)>0\right\} . \mathrm{S}_{o}^{\nu}$ is nothing but the fine support of $\nu$, i.e., the topological support of $\nu$ with respect to the fine topology of $\mathbf{X}$. Let $(\check{\mathbf{X}}, \nu)$ be the time changed process of $\mathbf{X}$ by $A_{t}^{\nu}$ and $(\check{\mathcal{E}}, \check{\mathcal{F}})$ the associated Dirichlet form on $L^{2}\left(\mathrm{~S}^{\nu} ; \nu\right)$, where $\mathrm{S}^{\nu}$ is the support of $\nu$. It is known that $(\check{\mathcal{E}}, \check{\mathcal{F}})$ is a regular Dirichlet form having

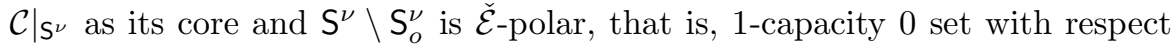
to $(\check{\mathcal{E}}, \check{\mathcal{F}})$. The life time of $(\check{\mathbf{X}}, \nu)$ is given by $A_{\zeta}^{\nu}$. Let $C^{\nu}: 2^{E} \rightarrow[0,+\infty]$ be the weighted 1 -capacity with respect to $(\check{\mathcal{E}}, \check{\mathcal{F}})$, that is, for an open subset $G$ of $E$, we define

$$
C^{\nu}(G):=\inf \left\{\check{\mathcal{E}}_{1}(f, f) \mid f \in \check{\mathcal{F}}, f \geq \check{R}_{1} \varphi \nu \text {-a.e. on } G\right\}
$$

and for arbitrary subset $A$ of $E$

$$
C^{\nu}(A):=\inf \left\{C^{\nu}(G) \mid A \subset G, G \text { is an open subset of } E\right\},
$$

where $\check{R}_{1} \varphi(x):=\mathbf{E}_{x}\left[\int_{0}^{\infty} e^{-A_{t}^{\nu}} \varphi\left(X_{t}\right) \mathrm{d} A_{t}^{\nu}\right]$ is the 1-order resolvent of a $\nu$-a.e. strictly positive bounded function $\varphi \in L^{1}(E ; \nu)$ under $(\check{\mathbf{X}}, \nu)$ and $\check{\mathcal{E}}_{1}(f, f):=$ $\check{\mathcal{E}}(f, f)+\int_{E} f^{2} \mathrm{~d} \nu$. We emphasize that $C^{\nu}$ is defined to be an outer capacity on $E$. By definition, $C^{\nu}\left(E \backslash \mathrm{S}^{\nu}\right)=0$. Note that $C^{\nu}(E) \leq \check{\mathcal{E}}_{1}\left(\check{R}_{1} \varphi, \check{R}_{1} \varphi\right)=$ $\int_{E} \varphi(x) \check{R}_{1} \varphi(x) \nu(\mathrm{d} x)<\infty$ always holds and $C^{\nu}$ is tight in the sense that there exists an increasing sequence $\left\{K_{n}\right\}$ of compact subsets of $\mathrm{S}^{\nu}$ such that $\lim _{n \rightarrow \infty} C^{\nu}\left(\mathrm{S}^{\nu} \backslash K_{n}\right)=0$ equivalently $\lim _{n \rightarrow \infty} C^{\nu}\left(E \backslash K_{n}\right)=0$. Hence, any quasi closed set with respect to $C^{\nu}$ is quasi compact in the sense of Fuglede [18, Lemma 2.2]. By [18, Theorem 2.10], any decreasing sequence $\left\{A_{n}\right\}$ of quasi closed subsets of $E$ with respect to $C^{\nu}$ satisfies

$$
C^{\nu}\left(\bigcap_{n=1}^{\infty} A_{n}\right)=\inf _{n \in \mathbb{N}} C^{\nu}\left(A_{n}\right) .
$$

Now we introduce some new classes of (semi-) Green-tight measures of (extended) Kato class:

Definition 4.2 (Natural (semi-)Green-tight measures of (extended) Kato class). Let $\alpha \geq 0$ and $\nu \in S_{1}(\mathbf{X})$. 
(1) $\nu$ is said to be an $\alpha$-order natural Green-tight measure of Kato class with respect to $\mathbf{X}$ if $\nu \in S_{D}^{1}(\mathbf{X})\left(\nu \in S_{D_{0}}^{1}(\mathbf{X})\right.$ for $\left.\alpha=0\right)$ and for any $\varepsilon>0$ there exist a closed subset $K=K(\varepsilon)$ of $E$ and a constant $\delta>0$ such that for all $\nu$-measurable subset $B \subset K$ with $C^{\nu}(B)<\delta$,

$$
\sup _{x \in E} \mathbf{E}_{x}\left[\int_{0}^{\tau_{B \cup K^{c}}} e^{-\alpha t} \mathrm{~d} A_{t}^{\nu}\right]<\varepsilon .
$$

(2) $\nu$ is said to be a 0-order natural semi-Green-tight measure of extended Kato class with respect to $\mathbf{X}$ if $\nu \in S_{D_{0}}^{1}(\mathbf{X})$ and there exist a closed subset $K$ of $E$ and a constant $\delta>0$ such that for all $\nu$-measurable subset $B \subset K$ with $C^{\nu}(B)<\delta$,

$$
\sup _{x \in E} \mathbf{E}_{x}\left[A_{\tau_{B \cup K^{c}}}^{\nu}\right]<1
$$

In view of the resolvent equation, for positive $\alpha$, the $\alpha$-order natural Greentightness is independent of the choice of $\alpha>0$. Let us denote by $S_{\mathrm{NK}_{\infty}^{+}}^{1}(\mathbf{X})$ the family of positive order natural Green-tight measures of Kato class with respect to $\mathbf{X}$. The class $S_{\mathrm{NK}_{\infty}}^{1}(\mathbf{X})$ (resp. $S_{\mathrm{NK}_{1}}^{1}(\mathbf{X})$ ) is then used to denote the family of 0-order natural Green-tight measures of Kato class (resp. the family of 0-order natural semi-Green-tight measures of extended Kato class) with respect to $\mathbf{X}$. Similarly, as we remarked after Definition 4.1, the closed set $K$ appeared in Definition 4.2 can be taken to be compact, because the weighted 1-capacity $C^{\nu}$ is tight.

Since $\int_{B} g \mathrm{~d} \nu \leq C^{\nu}(B)$ holds for the $\nu$-a.e. strictly positive bounded function $g:=\left(\check{R}_{1} \varphi\right)^{2} \in L^{1}(E ; \nu)$, by [26, Lemma 4.2], we have $S_{\mathrm{CK}_{\infty}}^{1}(\mathbf{X}) \subset$ $S_{\mathrm{NK}_{\infty}}^{1}(\mathbf{X})$ and $S_{\mathrm{CK}_{1}}^{1}(\mathbf{X}) \subset S_{\mathrm{NK}_{1}}^{1}(\mathbf{X})$, hence $S_{\mathrm{CK}_{\infty}^{+}}^{1}(\mathbf{X}) \subset S_{\mathrm{NK}_{\infty}^{+}}^{1}(\mathbf{X})$.

$\mathbf{X}$ is said to be a Feller process or to have the Feller property if $P_{t}\left(C_{\infty}(E)\right) \subset C_{\infty}(E)$ for each $t>0$ and $\lim _{t \rightarrow 0}\left\|P_{t} f-f\right\|_{\infty}=0$ for $f \in$ $C_{\infty}(E)$. The next proposition asserts that $S_{\mathrm{NK}_{\infty}}^{1}(\mathbf{X})$ is not so wide.

Proposition 4.1. Suppose that (RSF) and the Feller property hold for $\mathbf{X}$. Then we have $S_{K_{\infty}}^{1}(\mathbf{X})=S_{\mathrm{CK}_{\infty}}^{1}(\mathbf{X})=S_{\mathrm{NK}_{\infty}}^{1}(\mathbf{X})$ and $S_{K_{\infty}^{+}}^{1}(\mathbf{X})=S_{\mathrm{CK}_{\infty}^{+}}^{1}(\mathbf{X})=$ $S_{\mathrm{NK}_{\infty}^{+}}^{1}(\mathbf{X})$.

Proof. By [26, Lemma 4.1], we know $S_{K_{\infty}}^{1}(\mathbf{X})=S_{\mathrm{CK}_{\infty}}^{1}(\mathbf{X})$, so it suffices to prove $S_{\mathrm{NK}_{\infty}}^{1}(\mathbf{X}) \subset S_{K_{\infty}}^{1}(\mathbf{X})\left(S_{\mathrm{NK}_{\infty}^{+}}^{1}(\mathbf{X}) \subset S_{K_{\infty}^{+}}^{1}(\mathbf{X})\right.$ can be similarly proved without transience). Since $\mathbf{X}$ is transient, there exists an $\mathfrak{m}$-a.e. strictly positive bounded $g \in L^{1}(E ; \mathfrak{m})$ such that $R g \in \mathcal{B}_{b}(E)$. Let $\mathbf{X}^{g \mathfrak{m}}$ be the subprocess killed by $\exp \left(-\int_{0}^{t} g\left(X_{s}\right) \mathrm{d} s\right)$. Then $\mathbf{X}^{g \mathfrak{m}}$ enjoys $(\mathbf{R S F})$ and it is a transient Feller process by Corollary 5.1 in [28], that is, $R_{\alpha}^{g \mathfrak{m}} f \in C_{\infty}(E)$ for $\alpha>0$ and $f \in C_{\infty}(E)$. Let $\left\|R_{\alpha}^{g \mathfrak{m}}\right\|_{L\left(C_{\infty}(E)\right)}$ be the operator norm for $R_{\alpha}^{g \mathfrak{m}}: C_{\infty}(E) \rightarrow C_{\infty}(E)$ defined by

$$
\left\|R_{\alpha}^{g \mathfrak{m}}\right\|_{L\left(C_{\infty}(E)\right)}:=\sup _{f \in C_{\infty}(E),\|f\|_{\infty}=1} \sup _{x \in E}\left|R_{\alpha}^{g \mathfrak{m}} f(x)\right| \text {. }
$$


Then one can prove $\left\|\alpha R_{\alpha}^{g \mathfrak{m}}\right\|_{L\left(C_{\infty}(E)\right)}<1$ as in the proof of [28, Theorem 7.1], which implies

$$
R^{g \mathfrak{m}} \mathbf{1}_{K}=\sum_{n=0}^{\infty}\left(\alpha R_{\alpha}^{g \mathfrak{m}}\right)^{n} R_{\alpha}^{g \mathfrak{m}} \mathbf{1}_{K} \in C_{\infty}(E) .
$$

Let $\left\|R^{g \mathfrak{m}} \circ g\right\|_{L\left(C_{\infty}(E)\right)}$ be the operator norm of $R^{g \mathfrak{m}} \circ g: C_{\infty}(E) \rightarrow C_{\infty}(E)$ defined by $\left(R^{g \mathfrak{m}} \circ g\right)(f):=R^{g \mathfrak{m}}(g f)$ for $f \in C_{\infty}(E)$. Then

$$
\begin{aligned}
\left\|R^{g \mathfrak{m}} \circ g\right\|_{L\left(C_{\infty}(E)\right)} & =\sup _{f \in C_{\infty}(E),\|f\|_{\infty}=1} \sup _{x \in E}\left|R^{g \mathfrak{m}} g f(x)\right| \\
& \leq \sup _{x \in E} \mathbf{E}_{x}\left[\int_{0}^{\infty} e^{-\int_{0}^{t} g\left(X_{s}\right) \mathrm{d} s} g\left(X_{t}\right) \mathrm{d} t\right] \\
& =1-\inf _{x \in E} \mathbf{E}_{x}\left[e^{-\int_{0}^{\infty} g\left(X_{s}\right) \mathrm{d} s}\right] \leq 1-e^{-\|R g\|_{\infty}}<1,
\end{aligned}
$$

where we use Jensen's inequality. Consequently

$$
R \mathbf{1}_{K}=\sum_{n=0}^{\infty}\left(R^{g \mathfrak{m}} \circ g\right)^{n} R^{g \mathfrak{m}} \mathbf{1}_{K} \in C_{\infty}(E)
$$

holds in view of the following resolvent equation

$$
R \mathbf{1}_{K}(x)=R^{g \mathfrak{m}} \mathbf{1}_{K}(x)+R^{g \mathfrak{m}}\left(g R \mathbf{1}_{K}\right)(x),
$$

which is a special case of [30, Lemma 4.1.1]. By [1, Proposition 3.4], under $R \mathbf{1}_{K} \in C_{\infty}(E)$, we can obtain

$$
\lim _{x \rightarrow \partial} \mathbf{P}_{x}\left(\sigma_{K}<\infty\right)=0 \text { for any compact subset } K \text { of } E .
$$

Though the underlying process in [1] is assumed to be a diffusion, the proof for (4.4) in [1] remains valid for general Feller processes. Suppose $\nu \in S_{\mathrm{NK}_{\infty}}^{1}(\mathbf{X})$. Let $\varepsilon>0$ and take a closed set $K$ with

$$
\sup _{x \in E} \mathbf{E}_{x}\left[A_{\tau_{K^{c}}}^{\nu}\right]<\varepsilon .
$$

We may assume that $K$ is compact by taking a smaller compact subset in a similar way to the last remark after Definition 4.1. By definition, $\nu \in S_{D_{0}}^{1}(\mathbf{X})$ and $\nu \in S_{K}^{1}(\mathbf{X})$ by [26, Lemma 4.3], in particular $\mathbf{1}_{K} \nu \in S_{K_{\infty}}^{1}(\mathbf{X})$. Owing to $(\mathbf{R S F})$ of $\mathbf{X}$, we can get $R \mathbf{1}_{K} \nu \in C_{\infty}(E)$ by [17, Lemma 2.3(5)]. Note that the assertion of [17, Lemma 2.3(5)] remains valid under (RSF) and the Feller property of X. From this and (4.5),

$$
\begin{aligned}
\lim _{x \rightarrow \partial} R \nu(x) & =\lim _{x \rightarrow \partial} R \mathbf{1}_{K^{c}} \nu(x) \\
& =\lim _{x \rightarrow \partial}\left(\mathbf{E}_{x}\left[A_{\tau_{K^{c}}}^{\nu}\right]+\mathbf{E}_{x}\left[R \mathbf{1}_{K^{c}} \nu\left(X_{\sigma_{K}}\right): \sigma_{K}<\infty\right]\right) \\
& \leq \varepsilon+\|R \nu\|_{\infty} \lim _{x \rightarrow \partial} \mathbf{P}_{x}\left(\sigma_{K}<\infty\right)=\varepsilon .
\end{aligned}
$$

Since $\varepsilon>0$ is arbitrary, we obtain $R \nu \in C_{\infty}(E)$. Therefore, we have $\nu \in$ $S_{K_{\infty}}^{1}(\mathbf{X})$. 
Recall that $\mathrm{S}_{o}^{\nu}$ is the fine support of $A_{t}^{\nu}$ for a smooth measure $\nu \in S_{1}(\mathbf{X})$. As a special case of Lemma 4.5 in [26], we have the following lemma.

Lemma 4.1 (cf. [26, Lemma 4.5]). Assume $\mu_{\langle u\rangle} \in S_{K}^{1}(\mathbf{X})$. Then the following hold:

(1) For $\nu \in S_{D}^{1}(\mathbf{X}), e^{-2 u} \nu \in S_{D}^{1}(\mathbf{U})$.

(2) For $\nu \in S_{K}^{1}(\mathbf{X}), e^{-2 u} \nu \in S_{K}^{1}(\mathbf{U})$.

(3) For $\nu \in S_{\mathrm{EK}}^{1}(\mathbf{X}), e^{-2 u} \nu \in S_{\mathrm{EK}}^{1}(\mathbf{U})$.

(4) For $\nu \in S_{K_{\infty}^{+}}^{1}(\mathbf{X}), e^{-2 u} \nu \in S_{K_{\infty}^{+}}^{1}(\mathbf{U})$.

(5) For $\nu \in S_{\mathrm{CK}_{\infty}^{+}}^{1}(\mathbf{X}), e^{-2 u} \nu \in S_{\mathrm{CK}_{\infty}^{+}}^{1}(\mathbf{U})$.

(6) Assume $\mu_{\langle u\rangle} \in S_{\mathrm{NK}_{\infty}}^{1}(\mathbf{X})$. Suppose that $\mathrm{S}_{o}^{\nu} \supset \mathrm{S}_{o}^{\mu_{\langle u\rangle}^{c}+N\left(e^{U}-U-1\right) \mu_{H}}$ or there exists a measure $\eta \in S_{\mathrm{NK}_{\infty}^{+}}^{1}(\mathbf{X})$ such that $\operatorname{supp}\left[A^{\eta}\right]=E$. Then, for $\nu \in$ $S_{\mathrm{NK}_{\infty}^{+}}^{1}(\mathbf{X})$ we have $e^{-2 u} \nu \in S_{\mathrm{NK}_{\infty}^{+}}^{1}(\mathbf{U})$.

(7) Assume $\mu_{\langle u\rangle} \in S_{\mathrm{NK}_{\infty}}^{1}(\mathbf{X})$. Then, for $\nu \in S_{\mathrm{NK}_{1}}^{1}\left(\mathbf{X}^{(1)}\right)$ we have $e^{-2 u} \nu \in$ $S_{\mathrm{NK}_{1}}^{1}\left(\mathbf{U}^{(1)}\right)$.

Proof. We only prove (7), because an extra assumption is imposed in [26, Lemma $4.5(7)$ ] as for (6). Since $\mathbf{X}$ is transient, there exists a strictly positive bounded $g \in L^{1}(E ; \mathfrak{m})$ such that $g \mathfrak{m} \in S_{D_{0}}^{1}(\mathbf{X})$ by [21]. Suppose $\nu \in$ $S_{\mathrm{NK}_{1}}^{1}\left(\mathbf{X}^{(1)}\right)$. By taking small $\beta>0$, we see $\eta=\nu+\beta g \mathfrak{m} \in S_{\mathrm{NK}_{1}}^{1}\left(\mathbf{X}^{(1)}\right)$, whose fine support coincides with $E$. Then $S_{0}^{\eta} \supset S_{0}^{\mu_{\langle u\rangle}^{c}+N\left(e^{U}-U-1\right) \mu_{H}}$ is automatically satisfied. Thus we have $e^{-2 u} \eta \in S_{\mathrm{NK}_{1}}^{1}\left(\mathbf{U}^{(1)}\right)$, hence $e^{-2 u} \nu \in S_{\mathrm{NK}_{1}}^{1}\left(\mathbf{U}^{(1)}\right)$.

Let $\mu$ be a signed measure such that $\mu=\mu_{1}-\mu_{2}$ with $\mu_{1} \in S_{\mathrm{NK}_{1}}^{1}(\mathbf{X})$ and $\mu_{2} \in S_{1}(\mathbf{X})$. Note that for such $\mu=\mu_{1}-\mu_{2}$ we know $\mu_{+} \in S_{1}(\mathbf{X})$ and $\mu_{-} \in$ $S_{1}(\mathbf{X})$, because of $\mu_{+} \leq \mu_{1}$ and $\mu_{-} \leq \mu_{2}$. Let $\tau_{t}$ be the right continuous inverse of $A_{t}^{\mu_{1}}, \tau_{t}:=\inf \left\{s>0 \mid A_{s}^{\mu_{1}}>t\right\}$ with the convention that $\inf \emptyset=\infty$. Let $F_{1}, F_{2}$ be nonnegative bounded symmetric functions on $E \times E$ described as in Section 1 satisfying $N\left(F_{1}+F_{2}\right) \mu_{H} \in S_{1}(\mathbf{X})$. We set $F:=F_{1}-F_{2}$ and $A_{t}^{F}:=\sum_{s \leq t} F\left(X_{s-}, X_{s}\right)$, the purely discontinuous additive functional with jump $\Delta A_{t}^{\bar{F}}=F\left(X_{t-}, X_{t}\right), t \in[0, \infty[$.

For an $\mathrm{AF} A$, we say that $(\mathbf{X}, A)$ is gaugeable if

$$
\sup _{x \in E} \mathbf{E}_{x}\left[\exp \left(A_{\zeta}\right)\right]<\infty \text {. }
$$

Let $\mathbf{X}^{*}=\left(\Omega, X_{t}, \mathbf{P}_{x}^{*}\right)$ be the subprocess killed by $e^{-A_{t}^{\mu_{2}}-A_{t}^{F_{2}}}$ for $\mu_{2}+$ $N\left(F_{2}\right) \mu_{H} \in S_{1}(\mathbf{X})$. By [33, Section 62], we have that for any stopping time $T$,

$$
\begin{aligned}
\mathbf{E}_{x}^{*} & {\left[e^{A_{T}^{\mu_{1}}+A_{T}^{F_{1}}}\right] } \\
& =\mathbf{E}_{x}\left[\int_{0}^{T} e^{A_{s}^{\mu_{1}}+A_{s}^{F_{1}}} \mathrm{~d}\left(-e^{-A_{s}^{\mu_{2}}-A_{s}^{F_{2}}}\right)+e^{A_{T}^{\mu_{1}}+A_{T}^{F_{1}}-A_{T}^{\mu_{2}}-A_{T}^{F_{2}}}\right]
\end{aligned}
$$




$$
\begin{aligned}
& =\mathbf{E}_{x}\left[\int_{0}^{T} e^{A_{s}^{\mu_{1}}+A_{s}^{F_{1}}} e^{-A_{s}^{\mu_{2}}-A_{s}^{F_{2}}} \mathrm{~d}\left(-A_{s}^{-\mu_{2}}+A_{s}^{e^{F_{2}}-1}\right)\right]+\mathbf{E}_{x}\left[e^{A_{T}^{\mu}+A_{T}^{F}}\right] \\
& =\mathbf{E}_{x}\left[\int_{0}^{T} e^{A_{s}^{\mu}+A_{s}^{F}} \mathrm{~d}\left(A_{s}^{\mu_{2}}+A_{s}^{e^{F_{2}}-1}\right)\right]+\mathbf{E}_{x}\left[e^{A_{T}^{\mu}+A_{T}^{F}}\right] \\
& \geq \mathbf{E}_{x}\left[e^{A_{T}^{\mu}+A_{T}^{F}}\right] .
\end{aligned}
$$

Note here that for any stopping time $T, T \wedge \zeta$ is the reduced time for $e^{-A_{t}^{\mu_{2}}-A_{t}^{F_{2}}}$ in the sense of [33, Section 62]. The following gauge theorem can be similarly proved in this generality (see Theorem 5.1 below).

TheOrem 4.1 (Gauge Theorem). Suppose that $\mu_{1}+N\left(e^{F_{1}}-1\right) \mu_{H} \in$ $S_{\mathrm{NK}_{1}}^{1}(\mathbf{X})$ and $\mu_{2}+N\left(F_{2}\right) \mu_{H} \in S_{1}(\mathbf{X})$ hold. Then the following are equivalent.

(1) $\left(\mathbf{X}, A^{\mu}+A^{F}\right)$ is gaugeable.

(2) $\mathbf{E}_{x}\left[\exp \left(A_{\zeta}^{\mu}+A_{\zeta}^{F}\right)\right]<\infty$ for some $x \in E$.

Proof. It suffices to prove that $g(x):=\mathbf{E}_{x}\left[\exp \left(A_{\zeta}^{\mu}+A_{\zeta}^{F}\right)\right]$ satisfies $\sup _{x \in E} g(x)<\infty$ or $g \equiv \infty$. The proof is quite similar with the proofs of [4, Theorem 2.13], [10, Theorem 2.6]. The fine continuity of $x \mapsto g(x)$ and the absorbing property of the set $O:=\{g<\infty\}$ under $\mathbf{P}_{x}$ can be proved in a similar way as in [4], [10]. So, it suffices to confirm that there are $N>0$ and $C>0$ such that

$$
O=\{g<\infty\}=\{g \leq C(1+N)\}
$$

Then $O$ is finely open and finely closed. Since $E$ is connected with respect to the fine topology under $(\mathbf{I})$ and $(\mathbf{A C})$, we have $O=E$ or $O=\emptyset$, which means the assertion. From now on, we prove (4.7) for some $C, N$. Since $\eta_{1}:=$ $\mu_{1}+N\left(e^{F_{1}}-1\right) \mu_{H} \in S_{\mathrm{NK}_{1}}^{1}(\mathbf{X})$, there exist a closed subset $K$ of $\mathrm{S}^{\eta_{1}}$ and $\delta>0$ such that for any subset $B \subset K$ with $C^{\eta_{1}}(B)<\delta, \sup _{x \in E} \mathbf{E}_{x}\left[A_{\tau_{B \cup K^{c}}}^{\eta_{1}}\right]<1-c$ for some $c \in] 0,1\left[\right.$. Here $C^{\eta_{1}}$ is the weighted 1-capacity with respect to the Dirichlet form $(\check{\mathcal{E}}, \check{\mathcal{F}})$ associated to the time changed process $\left(\check{\mathbf{X}}, \eta_{1}\right)$ from $\mathbf{X}$ and $S^{\eta_{1}}$ is the topological support of $\eta_{1}$. Note that any finely closed subset of $\mathrm{S}^{\eta_{1}}$ is quasi closed with respect to $C^{\eta_{1}}$. By applying [18, Theorem 2.10] to the decreasing sequence $\left\{B_{n}\right\}$ of quasi closed sets defined by $B_{n}:=\{x \in K \mid n \leq$ $g(x)<\infty\}$, we can take large $N \in \mathbb{N}$ so that $C^{\eta_{1}}\left(B_{N}\right)<\delta$. Khasminskii's lemma tells us that

$$
\begin{aligned}
C & :=\sup _{x \in E} \mathbf{E}_{x}\left[\exp \left(A_{\tau_{B_{N} \cup K^{c}}}^{\mu_{1}}+A_{\tau_{B_{N} \cup K^{c}}}^{F_{1}}\right)\right] \\
& =\sup _{x \in E} \mathbf{E}_{x}\left[\operatorname{Exp}\left(A^{\mu_{1}}+A^{F^{F_{1}}-1}\right)_{\tau_{B_{N} \cup K^{c}}}\right] \\
& \leq \frac{1}{1-\sup _{x \in E} \mathbf{E}_{x}\left[A_{\tau_{B_{N} \cup K^{c}}}^{\eta_{1}}<\frac{1}{c}\right.}
\end{aligned}
$$


where $\tau_{B}:=\inf \left\{t>0 \mid X_{t} \notin B\right\}$. By the formula

$$
\begin{aligned}
g(x)= & \mathbf{E}_{x}\left[\exp \left(A_{\tau_{B_{N} \cup K^{c}}}^{\mu}+A_{\tau_{B_{N} \cup K^{c}}}^{F}\right): \tau_{B_{N} \cup K^{c}}=\zeta\right] \\
& +\mathbf{E}_{x}\left[\exp \left(A_{\tau_{B_{N} \cup K^{c}}}^{\mu}+A_{\tau_{B_{N} \cup K^{c}}}^{F}\right) g\left(X_{\tau_{B_{N} \cup K^{c}}}\right): \tau_{B_{N} \cup K^{c}}<\zeta\right],
\end{aligned}
$$

we have $g(x) \leq C+C N$ for $x \in O \cap B_{N}$ in view of $X_{\tau_{B_{N} \cup K^{c}}} \in O \cap\left(K \backslash B_{N}\right)$ $\mathbf{P}_{x^{-}}$a.s. on $\left\{\tau_{B_{N} \cup K^{c}}<\zeta\right\}$ for $x \in O$, where we use the absorbing property of $O$. Moreover, $g(x) \leq N$ for $x \in O \backslash B_{N}$. Thus we obtain (4.7).

Let $\mu$ be a signed measure such that $\mu=\mu_{1}-\mu_{2}$ with $\mu_{1}, \mu_{2} \in S_{1}(\mathbf{X})$ and let $F_{1}, F_{2}$ be a function on $E \times E$ vanishing on the diagonal diag satisfying $N\left(F_{1}+F_{2}\right) \mu_{H} \in S_{1}(\mathbf{X})$. The following lemmas and theorem are modifications of Lemmas 2.7, 2.14, Theorems 2.8, 2.15, Corollaries 2.9, 2.16 and Theorems 2.10, 2.11 in [4]. The proofs are quite similar to those in [4].

Lemma 4.2 (cf. [4, Lemmas 2.7, 2.14, Theorems 2.8, 2.15, Corollaries 2.9, 2.16 and Theorem 2.10]). Set $A:=A^{\mu}+A^{F}$ and $e_{A}(t):=\exp \left(A_{t}\right)$. Suppose that $\mu_{1}+N\left(e^{F_{1}}-1\right) \mu_{H} \in S_{\mathrm{NK}_{1}}^{1}(\mathbf{X})$ and $\mu_{2}+N\left(F_{2}\right) \mu_{H} \in S_{D_{0}}^{1}(\mathbf{X})$ hold. Then we have the following:

(1) If $(\mathbf{X}, A)$ is gaugeable, then for any $\delta>0$ there is a constant $c(\delta)>0$ such that

$$
\left\|g_{A}\right\|_{\infty}^{-1} g_{A}(x) \leq \sum_{n=0}^{\infty} \mathbf{E}_{x}\left[e_{A}\left(\tau_{n \delta}\right): \tau_{n \delta}<\zeta\right] \leq c(\delta)<\infty \quad \text { for all } x \in E .
$$

Here $\tau_{t}:=\inf \left\{s>0 \mid A_{s}^{\eta}>t\right\}$ is the right continuous inverse of $A_{t}^{\eta}$ with $\eta=\mu_{1}+N\left(F_{1}\right) \mu_{H}$ and $g_{A}(x):=\mathbf{E}_{x}\left[e_{A}(\zeta)\right]$ is the gauge function for $A=$ $A^{\mu}+A^{F}$.

(2) The following are equivalent:

(a) $(\mathbf{X}, A)$ is gaugeable.

(b) $\mathbf{E}_{x}\left[\int_{0}^{\zeta} e_{A}(t) \mathrm{d}\left(A_{t}^{\mu_{1}}+A_{t}^{F_{1}}\right)\right]<\infty$ for some $x \in E$.

(c) $\sup _{x \in E} \mathbf{E}_{x}\left[\int_{0}^{\zeta} e_{A}(t) \mathrm{d}\left(A_{t}^{\mu_{1}}+A_{t}^{F_{1}}\right)\right]<\infty$.

(d) $\mathbf{E}_{x}\left[\sup _{t \in[0, \zeta]} e_{A}(t)\right]<\infty$ for some $x \in E$.

(e) $\sup _{x \in E} \mathbf{E}_{x}\left[\sup _{t \in[0, \zeta]} e_{A}(t)\right]<\infty$.

(f) $\left(\mathbf{X}^{*}, A^{\mu_{1}}+A^{F_{1}}\right)$ is gaugeable. Here $\mathbf{X}^{*}$ is the killed process of $\mathbf{X}$ by $e^{-A_{t}^{\mu_{2}}-A_{t}^{F_{2}}}$.

Proof. (1): The proof is similar to the proofs of [4, Lemma 2.14, Theorem 2.15 and Corollary 2.16] with some modifications under the conditions. We provide the proof for readers' convenience. Since the proof is the same for any $\delta>0$, we take $\delta=1$. Since $\mu_{1}+N\left(e^{F_{1}}-1\right) \mu_{H} \in S_{\mathrm{NK}_{1}}^{1}(\mathbf{X})$, there is $p>1$ such that $p \mu_{1}+N\left(e^{p F_{1}}-1\right) \mu_{H} \in S_{\mathrm{NK}_{1}}^{1}(\mathbf{X})$. Indeed, let $\left\{D_{n}\right\}$ be a decreasing sequence such that $\mathbf{P}_{x}\left(\lim _{n \rightarrow \infty} \sigma_{D_{n}} \geq \zeta\right)=1 \mathcal{E}$-q.e. $x \in \mathrm{S}_{o}^{(p-1) \mu_{1}+N\left(e^{p F_{1}}-1\right) \mu_{H}}$, 
which is equivalent to $\mathcal{E}$-q.e. $x \in \mathrm{S}_{o}^{\mu_{1}+N\left(e^{F_{1}}-1\right) \mu_{H}}$, because $\mathrm{S}_{o}^{\mu_{1}+\mu_{2}}=\mathrm{S}_{o}^{\mu_{1}} \cup$ $\mathrm{S}_{o}^{\mu_{2}}$, and $F_{1} \leq e^{F_{1}}-1 \leq F_{1} e^{\left\|F_{1}\right\|_{\infty}}$ and $p F_{1} \leq e^{p F_{1}}-1 \leq p F_{1} e^{p\left\|F_{1}\right\|_{\infty}}$ imply $\mathrm{S}_{o}^{N\left(F_{1}\right) \mu_{H}}=\mathrm{S}_{o}^{N\left(e^{F_{1}}-1\right) \mu_{H}}=\mathrm{S}_{o}^{N\left(e^{p F_{1}}-1\right) \mu_{H}}$. Since

$$
\begin{aligned}
& p \mu_{1}+N\left(e^{p F_{1}}-1\right) \mu_{H}-\mu_{1}-N\left(e^{F_{1}}-1\right) \mu_{H} \\
& \quad=(p-1) \mu_{1}+N\left(\left(e^{(p-1) F_{1}}-1\right) e^{F_{1}}\right) \mu_{H} \\
& \quad \leq(p-1) \mu_{1}+(p-1) e^{(p-1)\left\|F_{1}\right\|_{\infty}} N\left(F_{1}\right) \mu_{H},
\end{aligned}
$$

we have

$$
\begin{aligned}
\lim _{n \rightarrow \infty} \sup _{x \in E} \mathbf{E}_{x}\left[A_{\tau_{D_{n}}}^{p \mu_{1}+N\left(e^{p F_{1}}-1\right) \mu_{H}}\right] \\
\leq \lim _{n \rightarrow \infty} \sup _{x \in E} \mathbf{E}_{x}\left[(p-1) A_{\tau_{D_{n}}}^{\mu_{1}}+(p-1) e^{(p-1)\left\|F_{1}\right\|_{\infty}} A_{\tau_{D_{n}}}^{N\left(e^{F_{1}}-1\right) \mu_{H}}\right] \\
\quad+\lim _{n \rightarrow \infty} \sup _{x \in E} \mathbf{E}_{x}\left[A_{\tau_{D_{n}}}^{\mu_{1}+N\left(e^{F_{1}}-1\right) \mu_{H}}\right] \\
\leq(p-1)\left(\sup _{x \in E} \mathbf{E}_{x}\left[A_{\zeta}^{\mu_{1}}\right]+e^{(p-1)\left\|F_{1}\right\|_{\infty}} \sup _{x \in E} \mathbf{E}_{x}\left[A_{\zeta}^{N\left(e^{F_{1}}-1\right) \mu_{H}}\right]\right) \\
\quad+\lim _{n \rightarrow \infty} \sup _{x \in E} \mathbf{E}_{x}\left[A_{\tau_{D_{n}}}^{\mu_{1}+N\left(e^{F_{1}}-1\right) \mu_{H}}\right]<1
\end{aligned}
$$

for $p$ sufficiently close to 1 . For such $p>1$ satisfying $p \mu_{1}+N\left(e^{p F_{1}}-1\right) \mu_{H} \in$ $S_{\mathrm{NK}_{1}}^{1}(\mathbf{X})$, there exist a Borel set $K_{1}$ and a constant $\delta>0$ such that

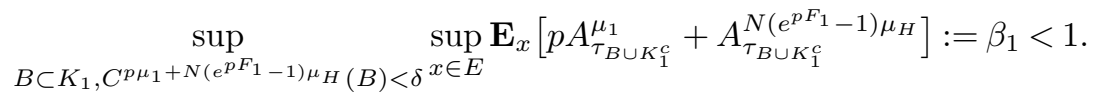

Since $(\mathbf{X}, A)$ is gaugeable, $g_{A}(x):=\mathbf{E}_{x}\left[e_{A}(\zeta)\right]$ is bounded and so

$$
\lim _{n \rightarrow \infty} \mathbf{E}_{x}\left[e_{A}(\zeta): \tau_{n}<\zeta\right]=0 \quad \text { for } x \in E .
$$

Thus for any given small $\varepsilon>0$ one can find an integer $N$ large enough and a closed subset $K \subset K_{1}$ such that

$$
\sup _{x \in K} \mathbf{E}_{x}\left[e_{A}(\zeta): \tau_{N}<\zeta\right]<\varepsilon,
$$

and $C^{p \mu_{1}+N\left(e^{p F_{1}}-1\right) \mu_{H}}\left(K_{1} \backslash K\right)<\delta$. Using Khasminskii's lemma,

$$
\begin{aligned}
\sup _{x \in E} \mathbf{E}_{x}\left[e_{A^{\mu_{1}}+A^{F_{1}}}\left(\sigma_{K}\right)^{p}\right] & \leq \sup _{x \in E} \mathbf{E}_{x}\left[\exp \left(p A_{\sigma_{K}}^{\mu_{1}}+p A_{\sigma_{K}}^{F_{1}}\right)\right] \\
& =\sup _{x \in E} \mathbf{E}_{x}\left[\operatorname{Exp}\left(A^{p \mu_{1}}+A^{e^{p F_{1}}-1}\right)_{\sigma_{K}}\right] \\
& \leq \frac{1}{1-\sup _{x \in E} \mathbf{E}_{x}\left[A_{\sigma_{K}}^{p \mu_{1}}+A_{\sigma_{K}}^{e^{p F_{1}}-1}\right]} \\
& \leq\left(1-\beta_{1}\right)^{-1},
\end{aligned}
$$


where $\sigma_{K}$ is the first hitting time of $K$ under $\mathbf{X}$. Now

$$
\begin{aligned}
\mathbf{E}_{x} & {\left[e_{A}(\zeta): \tau_{2 N}<\sigma_{K}\right] } \\
\leq & \mathbf{E}_{x}\left[e_{A}(\zeta): \tau_{N}<\sigma_{K}\right]+\mathbf{E}_{x}\left[e_{A}(\zeta): \sigma_{K} \leq \tau_{N}<\tau_{2 N}<\zeta\right] \\
\leq & \left\|g_{A}\right\|_{\infty} \mathbf{E}_{x}\left[e_{A}\left(\sigma_{K}\right): \tau_{N}<\sigma_{K}\right] \\
& \quad+\mathbf{E}_{x}\left[e_{A}\left(\sigma_{K}\right) \mathbf{E}_{X_{\sigma_{K}}}\left[e_{A}(\zeta): \tau_{N}<\zeta\right]: \sigma_{K}<\zeta\right] \\
:= & \mathrm{I}+\mathrm{II} .
\end{aligned}
$$

Denote by $\check{\mathbf{P}}_{x}^{\eta}$ the probability law for the time-changed process $(\check{\mathbf{X}}, \eta)$ and let $\check{\sigma}_{K}^{\eta}$ be the first hitting time of $K$ by $(\check{\mathbf{X}}, \eta)$. Then

$$
\check{\mathbf{E}}_{x}^{\eta}\left[\check{\sigma}_{K}^{\eta} \wedge \check{\zeta}^{\eta}\right]=\mathbf{E}_{x}\left[A_{\sigma_{K}}^{\eta}\right]
$$

By (4.6), we have

$$
\mathbf{E}_{x}\left[e_{A}\left(\sigma_{K}\right)^{p}\right]=\mathbf{E}_{x}\left[e_{p A}\left(\sigma_{K}\right)\right] \leq \mathbf{E}_{x}\left[e_{A^{p \mu_{1}}+A^{p F_{1}}}\left(\sigma_{K}\right)\right]=\mathbf{E}_{x}\left[e_{A^{\mu_{1}}+A^{F_{1}}}\left(\sigma_{K}\right)^{p}\right]
$$

for each $x \in E$. Let $q>1$ be such that $\frac{1}{p}+\frac{1}{q}=1$ and use Hölder's inequality,

$$
\begin{aligned}
\mathrm{I} & \leq\left\|g_{A}\right\|_{\infty}\left(\mathbf{E}_{x}\left[e_{A^{\mu_{1}}+A^{F_{1}}}\left(\sigma_{K}\right)^{p}\right]\right)^{\frac{1}{p}} \mathbf{P}_{x}\left(\tau_{N}<\sigma_{K}\right)^{\frac{1}{q}} \\
& \leq\left(1-\beta_{1}\right)^{-\frac{1}{p}}\left\|g_{A}\right\|_{\infty} \check{\mathbf{P}}_{x}^{\eta}\left(N<\check{\sigma}_{K}^{\eta}\right)^{\frac{1}{q}} \\
& \leq \frac{\left\|g_{A}\right\|_{\infty} \check{\mathbf{E}}_{x}^{\eta}\left[\check{\sigma}_{K}^{\eta} \wedge \check{\zeta}^{\eta}\right]^{\frac{1}{q}}}{\left(1-\beta_{1}\right)^{\frac{1}{p}} N^{\frac{1}{q}}} \leq \frac{\left\|g_{A}\right\|_{\infty} \mathbf{E}_{x}\left[A_{\sigma_{K}}^{\eta}\right]^{\frac{1}{q}}}{\left(1-\beta_{1}\right)^{\frac{1}{p}} N^{\frac{1}{q}}} \leq \frac{\left\|g_{A}\right\|_{\infty} \mathbf{E}_{x}\left[A_{\zeta}^{\eta}\right]^{\frac{1}{q}}}{\left(1-\beta_{1}\right)^{\frac{1}{p}} N^{\frac{1}{q}}} .
\end{aligned}
$$

So I $\rightarrow 0$ uniformly as $N \rightarrow \infty$. On the set $\left\{\sigma_{K}<\zeta\right\}, X_{\sigma_{K}} \in K$ and so

$$
\begin{aligned}
\mathrm{II} & \leq \varepsilon \mathbf{E}_{x}\left[e_{A}\left(\sigma_{K}\right): \sigma_{K}<\zeta\right] \leq \varepsilon \mathbf{E}_{x}\left[e_{A}\left(\sigma_{K}\right)\right] \\
& \leq \varepsilon \mathbf{E}_{x}\left[e_{A^{\mu_{1}}+A^{F_{1}}}\left(\sigma_{K}\right)\right] \leq \frac{\varepsilon}{1-\sup _{x \in E} \mathbf{E}_{x}\left[A_{\sigma_{K}}^{\mu_{1}}+A_{\sigma_{K}}^{F_{1}-1}\right]} \\
& \leq \frac{\varepsilon}{1-\beta_{1}},
\end{aligned}
$$

where we use (4.6). Thus for large $N, \sup _{x \in E} \mathbf{E}_{x}\left[e_{A}(\zeta): \tau_{N}<\zeta\right]$ is small. By Jensen's inequality, the gauge function $g_{A}$ is bounded below by a positive constant $c_{1}:=e^{-\sup _{x \in E} \mathbf{E}_{x}\left[A_{\zeta}^{\mu_{2}}+A_{\zeta}^{F_{2}}\right]}$. So

$$
\begin{aligned}
\mathbf{E}_{x}\left[e_{A}\left(\tau_{N}\right): \tau_{N}<\zeta\right] & \leq c_{1}^{-1} \mathbf{E}_{x}\left[e_{A}\left(\tau_{N}\right) g_{A}\left(X_{\tau_{N}}\right): \tau_{N}<\zeta\right] \\
& \leq c_{1}^{-1} \mathbf{E}_{x}\left[e_{A}(\zeta): \tau_{N}<\zeta\right] .
\end{aligned}
$$

Therefore, when $N$ is large enough,

$$
\sup _{x \in E} \mathbf{E}_{x}\left[e_{A}\left(\tau_{N}\right): \tau_{N}<\zeta\right]:=\lambda<1 .
$$

By the strong Markov property of $\mathbf{X}$,

$$
\sup _{x \in E} \mathbf{E}_{x}\left[e_{A}\left(\tau_{k N}\right): \tau_{k N}<\zeta\right]:=\lambda^{k}, \quad \text { for } k \geq 0 .
$$


By Schwarz inequality

$$
\begin{aligned}
\mathbf{E}_{x}\left[e_{A}\left(\tau_{j}\right): \tau_{j}<\zeta\right] \leq & \mathbf{E}_{x}\left[e_{A^{\mu_{1}}+A^{F_{1}}}\left(\tau_{j}\right): \tau_{j}<\zeta\right] \\
\leq & \mathbf{E}_{x}\left[\exp \left(2 A_{\tau_{j}}^{\mu_{1}}+2 \int_{0}^{\tau_{j}} N\left(F_{1}\right)\left(X_{s}\right) \mathrm{d} H_{s}\right): \tau_{j}<\zeta\right]^{\frac{1}{2}} \\
& \times \mathbf{E}_{x}\left[\exp \left(2 A_{\tau_{j}}^{F_{1}}-2 \int_{0}^{\tau_{j}} N\left(F_{1}\right)\left(X_{s}\right) \mathrm{d} H_{s}\right)\right]^{\frac{1}{2}} \\
\leq & e^{j},
\end{aligned}
$$

where we use $A_{\tau_{j}}^{\mu_{1}}+\int_{0}^{\tau_{j}} N\left(F_{1}\right)\left(X_{s}\right) \mathrm{d} H_{s}=j$ and the supermartingale property of

$$
t \mapsto \exp \left(2 A_{t}^{F_{1}}-2 \int_{0}^{t} N\left(F_{1}\right)\left(X_{s}\right) \mathrm{d} H_{s}\right)
$$

under $\mathbf{P}_{x}$. Then for $0 \leq j<N$,

$$
\begin{aligned}
\mathbf{E}_{x}\left[e_{A}\left(\tau_{j+k N}\right): \tau_{j+k N}<\zeta\right] & =\mathbf{E}_{x}\left[e_{A}\left(\tau_{j}\right) \mathbf{E}_{X_{\tau_{j}}}\left[e_{A}\left(\tau_{k N}\right): \tau_{k N}<\zeta\right]: \tau_{j}<\zeta\right] \\
& \leq \lambda^{k} \max _{0 \leq j<N} \mathbf{E}_{x}\left[e_{A}\left(\tau_{j}\right): \tau_{j}<\zeta\right] \leq \lambda^{k} e^{N-1}
\end{aligned}
$$

All these inequalities lead to

$$
\begin{aligned}
\sum_{n=0}^{\infty} \mathbf{E}_{x}\left[e_{A}\left(\tau_{n}\right): \tau_{n}<\zeta\right] & =\sum_{k=0}^{\infty} \sum_{j=0}^{N-1} \mathbf{E}_{x}\left[e_{A}\left(\tau_{j+k N}\right): \tau_{j+k N}<\zeta\right] \\
& =N e^{N-1} \sum_{k=0}^{\infty} \lambda^{k}=\frac{N e^{N-1}}{1-\lambda}<\infty
\end{aligned}
$$

Finally, we have, by the strong Markov property of $\mathbf{X}$,

$$
\begin{aligned}
g_{A}(x) & =\sum_{n=0}^{\infty} \mathbf{E}_{x}\left[e_{A}(\zeta): \tau_{n}<\zeta \leq \tau_{n+1}\right] \\
& \leq \sum_{n=0}^{\infty} \mathbf{E}_{x}\left[e_{A}\left(\tau_{n}\right) g_{A}\left(X_{\tau_{n}}\right): \tau_{n}<\zeta\right] \\
& \leq\left\|g_{A}\right\|_{\infty} \sum_{k=0}^{\infty} \mathbf{E}_{x}\left[e_{A}\left(\tau_{n}\right): \tau_{n}<\zeta\right]
\end{aligned}
$$

(2): The proof for the equivalences among (a)-(e) of (2) through (1) is quite similar to the proof of [4, Theorem 2.15 and Corollary 2.16]. We only prove the equivalence $(\mathrm{c}) \Longleftrightarrow(\mathrm{f})$. By [33, Section 62], we have

$$
\mathbf{E}_{x}^{*}\left[e^{A_{\zeta}^{\mu_{1}}+A_{\zeta}^{F_{1}}}\right]=\mathbf{E}_{x}\left[\int_{0}^{\zeta} e^{A_{s}^{\mu_{1}}+A_{s}^{F_{1}}} \mathrm{~d}\left(-e^{-A_{s}^{\mu_{2}}-A_{s}^{F_{2}}}\right)\right]+\mathbf{E}_{x}\left[e^{A_{\zeta}^{\mu}+A_{\zeta}^{F}}\right]
$$


Using Itô's formula for semimartingale, we see

$$
\begin{aligned}
e^{A_{t}^{\mu}+A_{t}^{F}}-1= & \int_{0}^{t} e^{A_{s}^{\mu_{1}}+A_{s}^{F_{1}}} \mathrm{~d}\left(e^{-A_{s}^{\mu_{2}}-A_{s}^{F_{2}}}\right)+\int_{0}^{t} e^{-A_{s}^{\mu_{2}}-A_{s}^{F_{2}}} \mathrm{~d}\left(e^{A_{s}^{\mu_{1}}+A_{s}^{F_{1}}}\right) \\
& +\sum_{s \leq t} \Delta e^{A_{s}^{\mu_{1}}+A_{s}^{F_{1}}} \Delta e^{-A_{s}^{\mu_{2}}-A_{s}^{F_{2}}} \\
= & \int_{0}^{t} e^{A_{s}^{\mu_{1}}+A_{s}^{F_{1}}} \mathrm{~d}\left(e^{-A_{s}^{\mu_{2}}-A_{s}^{F_{2}}}\right)+\int_{0}^{t} e^{-A_{s}^{\mu_{2}}-A_{s}^{F_{2}}} \mathrm{~d}\left(e^{A_{s}^{\mu_{1}}+A_{s}^{F_{1}}}\right) \\
& +\sum_{s \leq t} e^{A_{s-}^{F}}\left(e^{F_{1}\left(X_{s-}, X_{s}\right)}-1\right)\left(e^{-F_{2}\left(X_{s-}, X_{s}\right)}-1\right) \\
\leq & \int_{0}^{t} e^{A_{s}^{\mu_{1}}+A_{s}^{F_{1}}} \mathrm{~d}\left(e^{-A_{s}^{\mu_{2}}-A_{s}^{F_{2}}}\right)+\int_{0}^{t} e^{-A_{s}^{\mu_{2}}-A_{s}^{F_{2}}} \mathrm{~d}\left(e^{A_{s}^{\mu_{1}}+A_{s}^{F_{1}}}\right) .
\end{aligned}
$$

From (4.8), we have

$$
\begin{aligned}
\mathbf{E}_{x}^{*}\left[e^{A_{\zeta}^{\mu_{1}}+A_{\zeta}^{F_{1}}}\right] & \leq \mathbf{E}_{x}\left[\int_{0}^{\zeta} e^{-A_{s}^{\mu_{2}}-A_{s}^{F_{2}}} \mathrm{~d}\left(e^{A_{s}^{\mu_{1}}+A_{s}^{F_{1}}}\right)\right]+1 \\
& =1+\mathbf{E}_{x}\left[\int_{0}^{\zeta} e_{A}(s) \mathrm{d}\left(A_{s}^{\mu_{1}}+A_{s}^{1-e^{-F_{1}}}\right)\right] \\
& \leq 1+\mathbf{E}_{x}\left[\int_{0}^{\zeta} e_{A}(s) \mathrm{d}\left(A_{s}^{\mu_{1}}+A_{s}^{F_{1}}\right)\right]
\end{aligned}
$$

If $(\mathbf{X}, A)$ is gaugeable, then $\left(\mathbf{X}^{*}, A^{\mu_{1}}+A^{F_{1}}\right)$ is gaugeable by $(\mathrm{c})$, and the converse is clear from (4.8).

REMARK 4.1. If $\mathfrak{m} \in S_{D_{0}}^{1}(\mathbf{X})$, under the same condition as in Lemma 4.2, without using time change, we have the following: Suppose that $(\mathbf{X}, A)$ is gaugeable with $A=A^{\mu}+A^{F}$ and $e_{A}(t):=\exp \left(A_{t}\right)$. Then, for any $\delta>0$, there is a constant $c(\delta)>0$ such that

$$
\left\|g_{A}\right\|_{\infty}^{-1} g_{A}(x) \leq \sum_{n=0}^{\infty} \mathbf{E}_{x}\left[e_{A}(n \delta): n \delta<\zeta\right] \leq c(\delta)<\infty \quad \text { for all } x \in E .
$$

The proof is very similar to the proof of Lemma $4.2(1)$ by changing $\tau_{k}$ to $k$ and replacing the estimate $\max _{0 \leq j<N} \mathbf{E}_{x}\left[e_{A}\left(\tau_{j}\right): \tau_{j}<\zeta\right] \leq e^{N-1}$ with $\max _{0 \leq j<N} \mathbf{E}_{x}\left[e_{A}(j): j<\zeta\right] \leq \sup _{x \in E} \mathbf{E}_{x}\left[\sup _{t \in[0, \zeta]} e_{A}(t)\right]<\infty$ (cf. the proof of Lemma 9 in [16]).

By Remark 4.1, we can prove the following lemma.

Lemma 4.3 (cf. [4, Theorem 2.11], [16, Lemma 9]). Suppose $\mathfrak{m} \in S_{D_{0}}^{1}(\mathbf{X})$, $\mu_{1}+N\left(e^{F_{1}}-1\right) \mu_{H} \in S_{\mathrm{NK}_{1}}^{1}(\mathbf{X})$ and $\mu_{2}+N\left(F_{2}\right) \mu_{H} \in S_{D_{0}}^{1}(\mathbf{X})$. Set $A:=A^{\mu}+$ $A^{F}$ and $e_{A}(t):=\exp \left(A_{t}\right)$. Then the following are equivalent: 
(1) $(\mathbf{X}, A)$ is gaugeable.

(2) For some $\delta>0$ and some $x \in E$,

$$
\sum_{n=0}^{\infty} \mathbf{E}_{x}\left[e_{A}(n \delta): n \delta<\zeta\right]<\infty
$$

(3) For all $\delta>0$ and all $x \in E$, (4.9) is true.

(4) For some $x \in E, \mathbf{E}_{x}\left[\int_{0}^{\zeta} e_{A}(t) \mathrm{d} t\right]<\infty$.

(5) $\sup _{x \in E} \mathbf{E}_{x}\left[\int_{0}^{\zeta} e_{A}(t) \mathrm{d} t\right]<\infty$.

(6) There exists some $t>0$ such that $\sup _{x \in E} \mathbf{E}_{x}\left[e_{A}(t): t<\zeta\right]<\infty$.

(7) There are constants $C>0$ and $b>0$ such that $\sup _{x \in E} \mathbf{E}_{x}\left[e_{A}(t): t<\zeta\right] \leq$ $C e^{-b t}$ for all $t>0$.

Proof. We only show that under the gaugeability of $(\mathbf{X}, A)$ there exists $C>0$ such that

$$
\mathbf{E}_{x}\left[\int_{0}^{\zeta} e_{A}(t) \mathrm{d} t\right] \leq C \delta \sum_{n=0}^{\infty} \mathbf{E}_{x}\left[e_{A}(n \delta): n \delta<\zeta\right]
$$

Note that the gaugeability $(\mathbf{X}, A)$ is equivalent to

$$
C:=\sup _{y \in E} \mathbf{E}_{y}\left[\sup _{t \in[0, \zeta]} e_{A}(t)\right]<\infty
$$

by Lemma $4.2(2)$. Then

$$
\begin{aligned}
\mathbf{E}_{x}\left[\int_{0}^{\zeta} e_{A}(t) \mathrm{d} t\right] & =\mathbf{E}_{x}\left[\sum_{n=0}^{\infty} \int_{n \delta \wedge \zeta}^{(n+1) \delta \wedge \zeta} e_{A}(t) \mathrm{d} t\right] \\
& =\sum_{n=0}^{\infty} \mathbf{E}_{x}\left[\int_{n \delta}^{(n+1) \delta \wedge \zeta} e_{A}(t) \mathrm{d} t: n \delta<\zeta\right] \\
& =\sum_{n=0}^{\infty} \mathbf{E}_{x}\left[e_{A}(n \delta) \mathbf{E}_{X_{n \delta}}\left[\int_{0}^{\delta \wedge \zeta} e_{A}(t) \mathrm{d} t\right]: n \delta<\zeta\right] \\
& \leq C \delta \sum_{n=0}^{\infty} \mathbf{E}_{x}\left[e_{A}(n \delta): n \delta<\zeta\right]
\end{aligned}
$$

Recall that the quadratic form $(\mathcal{Q}, \mathcal{F})$ defined in $(1.2)$. We write the quadratic form $\mathcal{Q}$ by $\mathcal{Q}^{*}$ for the case $u=0$. Using Lemma 4.3, we can prove the following theorem, whose proof is similar to that in [4, Theorem 2.12].

ThEOREM 4.2 (cf. [4, Theorem 2.12]). Suppose that $\mathfrak{m} \in S_{D_{0}}^{1}(\mathbf{X})$ and $\mathfrak{m}(E)<\infty$. Assume $\mu_{1}+N\left(e^{F_{1}}-1\right) \mu_{H} \in S_{\mathrm{NK}_{1}}^{1}(\mathbf{X})$ and $\mu_{2}+N\left(F_{2}\right) \mu_{H} \in$ $S_{D_{0}}^{1}(\mathbf{X})$. Set $A:=A^{\mu}+A^{F}$ and $e_{A}(t):=\exp \left(A_{t}\right)$. Then $(\mathbf{X}, A)$ is gaugeable 
if and only if

$$
\lambda^{\mathcal{Q}^{*}}(\mathfrak{m}):=\inf \left\{\mathcal{Q}^{*}(f, f) \mid f \in \mathcal{C}, \int_{E} f^{2} \mathrm{dm}=1\right\}>0 .
$$

Consider the non-local Feynman-Kac transforms by the additive functionals $A:=N^{u}+A^{\mu}+A^{F}$ of the form (1.1). Moreover, by (3.4), we see for all $t \in[0, \infty[$,

$$
e_{A}(t)=U_{t} e^{u\left(X_{t}\right)-u\left(X_{0}\right)} \exp \left(A_{t}^{\bar{\nu}}+A_{t}^{F}\right),
$$

where $\bar{\nu}=\bar{\nu}_{1}-\bar{\nu}_{2}$ and $\bar{\nu}_{1}:=\mu_{1}+N\left(e^{U}-U-1\right) \mu_{H}+\frac{1}{2} \mu_{\langle u\rangle}^{c}$ and $\bar{\nu}_{2}:=\mu_{2}$. Hence for $x \in E$ and $f \in \mathcal{B}_{+}(E)$,

$$
\mathbf{E}_{x}\left[e_{A}(t) f\left(X_{t}\right)\right]=e^{-u(x)} \mathbf{E}_{x}^{U}\left[\exp \left(A_{t}^{\bar{\nu}}+A_{t}^{F}\right)\left(e^{u} f\right)\left(\widetilde{X}_{t}\right)\right] .
$$

Lemma 4.4 (cf. Lemma 4.9 in [26]). Suppose that $\mu_{\langle u\rangle} \in S_{D_{0}}^{1}(\mathbf{X})$. Then

$$
\mathbf{E}_{x}^{U}\left[\exp \left(A_{\zeta}^{\bar{\nu}}+A_{\zeta}^{F}\right)\right]=e^{u(x)} \mathbf{E}_{x}\left[e^{-u\left(X_{\zeta-}\right)} e_{A}(\zeta)\right] .
$$

Proof. The proof is quite similar to that of [26, Lemma 4.9]. We omit the detail.

\section{Analytic characterizations for gaugeability}

In this section, we give several analytic characterizations of gaugeability for our generalized Feynman-Kac transforms $e_{A}(t)$.

Define the signed measure $\bar{\mu}:=\bar{\mu}_{1}-\bar{\mu}_{2}$ by

$$
\bar{\mu}_{1}:=N(V) \mu_{H}+\mu_{1}+\frac{1}{2} \mu_{\langle u\rangle}^{c}, \quad \bar{\mu}_{2}:=N\left(F_{2}\right) \mu_{H}+\mu_{2}
$$

for the nonnegative function $V(x, y):=\left(G^{u}-F^{u}+F_{1}\right)(x, y)$. Similarly, define the signed measure $\bar{\mu}^{*}:=\bar{\mu}_{1}^{*}-\bar{\mu}_{2}^{*}$ by

$$
\bar{\mu}_{1}^{*}:=N\left(V^{*}\right) \mu_{H}+\mu_{1}+\frac{1}{2} \mu_{\langle u\rangle}^{c}, \quad \bar{\mu}_{2}^{*}:=\mu_{2}
$$

for $V^{*}(x, y):=V_{u, F_{1}}(x, y)$, that is, $V^{*}(x, y)=\left(G_{1}^{u}-F_{1}^{u}+F_{1}\right)(x, y)$. Then we see $\bar{\mu}=N\left(V-F_{2}\right) \mu_{H}+\mu+\frac{1}{2} \mu_{\langle u\rangle}^{c}, \bar{\mu}^{*}=N\left(V^{*}\right) \mu_{H}+\mu+\frac{1}{2} \mu_{\langle u\rangle}^{c}$ and $V^{*}-V=$ $G_{1}^{u}-G^{u}-F_{2}$, and thus $\bar{\mu}^{*}-\bar{\mu}=N\left(G_{1}^{u}-G^{u}\right) \mu_{H} \geq 0$. Note that $\bar{\mu}_{i}=\bar{\mu}_{i}^{*}=\bar{\nu}_{i}$ $(i=1,2)$ and $V=V^{*}$ for the case $F_{1}=F_{2}=0$.

Note that if $\nu \in S_{D}^{1}(\mathbf{X})\left(\right.$ resp. $\left.\nu \in S_{D_{0}}^{1}(\mathbf{X})\right)$, then $\int_{E} f^{2} \mathrm{~d} \nu \leq\left\|R_{1} \nu\right\|_{\infty} \mathcal{E}_{1}(f, f)$ for $f \in \mathcal{F}$ (resp. $\int_{E} f^{2} \mathrm{~d} \nu \leq\|R \nu\|_{\infty} \mathcal{E}(f, f)$ for $\left.f \in \mathcal{F}_{e}\right)$, hence $\mathcal{F} \subset L^{2}(E ; \nu)$ (resp. $\mathcal{F}_{e} \subset L^{2}(E ; \nu)$ ) from [36, Theorem 3.1]. The quadratic form $\mathcal{Q}$ on $\mathcal{F}$ defined in (1.2) is extended to $\mathcal{F}_{e} \times \mathcal{F}_{e}$ with the same expression (1.2) provided $\mu_{1}+N\left(F_{1}\right) \mu_{H}+\mu_{\langle u\rangle} \in S_{D_{0}}^{1}(\mathbf{X})$ and $\mu_{2}+N\left(F_{2}\right) \mu_{H} \in S_{D_{0}}^{1}(\mathbf{X})$. In view of (3.7), we have

$$
\mathcal{E}^{Z}\left(f e^{u}, f e^{u}\right)=\mathcal{E}^{Y}\left(f e^{u}, f e^{u}\right)+\int_{E} f^{2} N\left(G_{1}^{u}-G^{u}\right) \mathrm{d} \mu_{H} .
$$


It follows from (3.4), Theorem 3.2 and the Feynman-Kac formula that for $f \in \mathcal{F} \cap C_{0}(E)$, we have

$$
\begin{aligned}
\mathcal{E}^{Y}\left(f e^{u}, f e^{u}\right) & =\mathcal{Q}(f, f)+\int_{E} f^{2} \mathrm{~d}\left(\frac{1}{2} \mu_{\langle u\rangle}^{c}+\mu\right)+\int_{E} f^{2} N\left(V-F_{2}\right) \mathrm{d} \mu_{H} \\
& =\mathcal{Q}(f, f)+\int_{E} f^{2} \mathrm{~d} \bar{\mu},
\end{aligned}
$$

hence

$$
\begin{aligned}
\mathcal{E}^{Z}\left(f e^{u}, f e^{u}\right) & =\mathcal{Q}(f, f)+\int_{E} f^{2} \mathrm{~d} \bar{\mu}+\int_{E} f^{2} N\left(G_{1}^{u}-G^{u}\right) \mathrm{d} \mu_{H} \\
& =\mathcal{Q}(f, f)+\int_{E} f^{2} \mathrm{~d} \bar{\mu}^{*}
\end{aligned}
$$

Since $\mathbf{X}$ is transient, there exists an $\mathfrak{m}$-a.e. strictly positive bounded measurable function $g$ satisfying $g \mathfrak{m} \in S_{D_{0}}^{1}(\mathbf{X})$ (see Getoor [21]). We fix such a $g \mathfrak{m} \in S_{D_{0}}^{1}(\mathbf{X})$. Let $\left\{\tau_{t}^{g \mathfrak{m}}\right\}_{t \geq 0}$ be the right continuous inverse of the PCAF $A_{t}^{g \mathfrak{m}}:=\int_{0}^{t} g\left(X_{s}\right) \mathrm{d} s$, that is, $\tau_{t}^{g \mathfrak{m}}:=\inf \left\{s>0 \mid A_{s}^{g \mathfrak{m}}>t\right\}$. Let $(\check{\mathbf{X}}, g \mathfrak{m})$ be the time changed process of $\mathbf{X}$ by $A_{t}^{g \mathfrak{m}}$. Then $(\check{\mathbf{X}}, g \mathfrak{m})$ is $g \mathfrak{m}$-symmetric Hunt process on $E$ satisfying $(\mathbf{I})$ and $(\mathbf{A C}$ ) (see [35, Theorems 8.2 and 8.5] for the stability of $(\mathbf{I}))$.

LEMMA 5.1. We have the following:

(1) Any $\nu \in S_{\mathrm{NK}_{\infty}}^{1}(\mathbf{X}) \quad\left(\right.$ resp. $\nu \in S_{\mathrm{NK}_{1}}^{1}(\mathbf{X})$ ) satisfies $\nu \in S_{\mathrm{NK}_{\infty}}^{1}(\check{\mathbf{X}}, g \mathfrak{m})$ (resp. $\left.\nu \in S_{\mathrm{NK}_{1}}^{1}(\check{\mathbf{X}}, g \mathfrak{m})\right)$. Any $\nu \in S_{D_{0}}^{1}(\mathbf{X})$ satisfies $\nu \in S_{D_{0}}^{1}(\check{\mathbf{X}}, g \mathfrak{m})$.

(2) The gauge function $g_{A}(x)=\mathbf{E}_{x}\left[e_{A}(\zeta)\right]$ is invariant under the time changed process $(\check{\mathbf{X}}, g \mathfrak{m})$ in the sense that

$$
\mathbf{E}_{x}\left[e_{A}(\zeta)\right]=\mathbf{E}_{x}\left[e_{A_{\tau} g \mathfrak{m}}\left(A_{\zeta}^{g \mathfrak{m}}\right)\right]
$$

Here $A_{\tau_{t}^{g \mathfrak{m}}}:=A_{\tau_{t}^{g \mathfrak{m}}}^{\mu}+A_{\tau_{t}^{g \mathfrak{m}}}^{F}+N_{\tau_{t}^{g \mathfrak{m}}}^{u}$ is the $A F$ under $(\check{\mathbf{X}}, g \mathfrak{m})$.

Proof. (2) is a trivial observation. So it suffices to prove (1). We prove only $\nu \in S_{\mathrm{NK}_{\infty}}^{1}(\mathbf{X})$ implies $\nu \in S_{\mathrm{NK}_{\infty}}^{1}(\check{\mathbf{X}}, g \mathfrak{m})$. Let us denote by $\check{R}(x, y)$ the 0 -order resolvent kernel with respect to $(\check{\mathbf{X}}, g \mathfrak{m})$. Then we see $\check{R}(x, y)=R(x, y)$ for $g \mathfrak{m}$-a.e. $y \in E$. Moreover, we have $\check{R}(x, y)=R(x, y)$ for $x, y \in E$ because $\check{R}(x, \cdot)$ and $R(x, \cdot)$ are finely continuous with respect to $(\check{\mathbf{X}}, g \mathfrak{m})$. Take any $\varepsilon>0$ and any closed set $K$. If $\nu \in S_{\mathrm{NK}_{\infty}}^{1}(\mathbf{X})$, then there exist $\delta>0$ and any Borel set $B \subset K$ with $C^{\nu}(B)<\delta$ we have

$$
\sup _{x \in E} \mathbf{E}_{x}\left[A_{\tau_{B \cup K^{c}}}^{\nu}\right]<\varepsilon
$$

which implies $\nu \in S_{\mathrm{NK}_{\infty}}^{1}(\check{\mathbf{X}}, g \mathfrak{m})$ where we use that the PCAF associated to $\nu$ under $(\check{\mathbf{X}}, g \mathfrak{m})$ is given by $A_{\tau_{t}^{g \mathfrak{m}}}^{\nu}([30$, Theorem 4.3.3]), the first exit time from $B \cup K^{c}$ under $(\check{\mathbf{X}}, g \mathfrak{m})$ is given by $A_{\tau_{B \cup K^{c}}}^{g \mathfrak{m}}$, and $\tau_{A_{t}^{g \mathfrak{m}}}=t \wedge \zeta$. In particular, we have $g \mathfrak{m} \in S_{D_{0}}^{1}(\check{\mathbf{X}}, g \mathfrak{m}), \mu_{1}+N\left(e^{F_{1}}-1\right) \mu_{H} \in S_{\mathrm{NK}_{1}}^{1}(\check{\mathbf{X}}, g \mathfrak{m})$, $\mu_{\langle u\rangle} \in S_{\mathrm{NK}_{\infty}}^{1}(\check{\mathbf{X}}, g \mathfrak{m})$ and $\mu_{2}+N\left(F_{2}\right) \mu_{H} \in S_{D_{0}}^{1}(\check{\mathbf{X}}, g \mathfrak{m})$. 
For $\eta \in S_{D}^{1}(\mathbf{X})$, we set $\lambda^{\mathcal{Q}}(\eta)$ as defined in (1.3).

Proposition 5.1. Suppose that $\bar{\mu}_{1}^{*} \in S_{\mathrm{NK}_{1}}^{1}(\mathbf{X}), \mu_{\langle u\rangle} \in S_{\mathrm{NK}_{\infty}}^{1}(\mathbf{X})$ and $\mu_{2}+$ $N\left(F_{2}\right) \mu_{H} \in S_{D_{0}}^{1}(\mathbf{X})$. Then $\lambda^{\mathcal{Q}}\left(\bar{\mu}_{1}\right)>0$ implies that (1.1) is gaugeable.

Proof. It is proved in [26, Lemma 5.1] that $\lambda^{\mathcal{Q}}\left(\bar{\mu}_{1}\right)>0$ is equivalent to $\lambda^{\mathcal{Q}}\left(\bar{\mu}_{1}^{*}\right)>0$. So it suffices to prove that $\lambda^{\mathcal{Q}}\left(\bar{\mu}_{1}^{*}\right)>0$ implies the gaugeability of (1.1). Moreover, we may assume $\mathfrak{m} \in S_{D_{0}}^{1}(\mathbf{X})$ and $\mathfrak{m}(E)<\infty$ in view of Lemma 5.1. Recall $\bar{\nu}_{1}:=\mu_{1}+N\left(e^{U}-U-1\right) \mu_{H}+\frac{1}{2} \mu_{\langle u\rangle}^{c}$ and $\bar{\nu}_{2}:=\mu_{2}$. Then we easily see $\bar{\nu}_{1}+N\left(e^{U}\left(e^{F_{1}}-1\right)\right) \mu_{H} \in S_{\mathrm{NK}_{1}}^{1}(\mathbf{X})$ and $\bar{\nu}_{2}+N\left(e^{U} F_{2}\right) \mu_{H} \in S_{D_{0}}^{1}(\mathbf{X})$. By assumption $\mathfrak{m} \in S_{D_{0}}^{1}(\mathbf{X})$, we see that $\beta \sup _{x \in E} \mathbf{E}_{x}[\zeta]<1$ for small $\beta>0$, hence not only $\eta_{1}=\beta \mathfrak{m}+\bar{\nu}_{1} \in S_{\mathrm{NK}_{1}}^{1}(\mathbf{X})\left(\right.$ resp. $\left.\eta_{2}=\beta \mathfrak{m}+\bar{\nu}_{2} \in S_{D_{0}}^{1}(\mathbf{X})\right)$ but also $\eta_{1}+N\left(e^{U}\left(e^{F_{1}}-1\right)\right) \mu_{H} \in S_{\mathrm{NK}_{1}}^{1}(\mathbf{X})\left(\right.$ resp. $\left.\eta_{2}+N\left(e^{U} F_{2}\right) \mu_{H} \in S_{D_{0}}^{1}(\mathbf{X})\right)$ holds for such $\beta>0$. We have that $e^{-2 u} \eta_{1}+e^{-u} N\left(e^{-u}\left(e^{F_{1}}-1\right)\right) \mu_{H} \in$ $S_{\mathrm{NK}_{1}}^{1}\left(\mathbf{U}^{(\beta)}\right)$ and $e^{-2 u} \eta_{2}+e^{-u} N\left(e^{-u} F_{2}\right) \mu_{H} \in S_{D_{0}}^{1}\left(\mathbf{U}^{(\beta)}\right)$ hold by Lemma 4.1, because $\eta_{1}+N\left(e^{U}\left(e^{F_{1}}-1\right)\right) \mu_{H} \in S_{\mathrm{NK}_{1}}^{1}(\mathbf{X}), \eta_{2}+N\left(e^{U} F_{2}\right) \mu_{H} \in S_{D_{0}}^{1}(\mathbf{X})$ and $\eta_{1}$ has full fine support. Since $e^{-2 u} \mathfrak{m} \in S_{D}^{1}(\mathbf{U})=S_{D_{0}}^{1}\left(\mathbf{U}^{(\beta)}\right)$ by Lemma 4.1(1) and $e^{-2 u} \mathfrak{m}(E)<\infty$, we can apply Theorem 4.2 to $\mathbf{U}^{(\beta)}$. By (4.11), the gaugeability $\sup _{x \in E} \mathbf{E}_{x}\left[e_{A}(\zeta)\right]<\infty$ is equivalent to $\sup _{x \in E} \mathbf{E}_{x}^{U}\left[e^{A_{\zeta}^{\bar{\nu}}+A_{\zeta}^{F}}\right]<\infty$, which follows from

$$
\sup _{x \in E} \mathbf{E}_{x}^{U^{(\beta)}}\left[e^{\beta \zeta+A_{\zeta}^{\bar{\nu}}+A_{\zeta}^{F}}\right]<\infty .
$$

Indeed, [33, Section 62, (62.13)] with the uniform integrability of $\left\{U_{t}\right\}_{t \in[0, \infty]}$ yields

$$
\begin{aligned}
& \mathbf{E}_{x}^{U^{(\beta)}}\left[e^{\beta \zeta+A_{\zeta}^{\bar{\nu}}+A_{\zeta}^{F}}\right] \\
& =\mathbf{E}_{x}^{U}\left[\int_{0}^{\zeta} e^{\beta s+A_{s}^{\bar{\nu}}+A_{s}^{F}} \mathrm{~d}\left(-e^{-\beta s}\right)+e^{\beta \zeta+A_{\zeta}^{\bar{\nu}}+A_{\zeta}^{F}} e^{-\beta \zeta}\right] \\
& =\beta \mathbf{E}_{x}^{U}\left[\int_{0}^{\zeta} e^{A_{s}^{\bar{\nu}}+A_{s}^{F}} \mathrm{~d} s\right]+\mathbf{E}_{x}^{U}\left[\exp \left(A_{\zeta}^{\bar{\nu}}+A_{\zeta}^{F}\right)\right] .
\end{aligned}
$$

Then, applying Theorem 4.2 to $\left(\mathcal{E}^{U^{(\beta)}}, \mathcal{F}^{U^{(\beta)}}\right)$ with $e^{-2 u} \eta_{1}+e^{-u} N\left(e^{-u}\left(e^{F_{1}}-\right.\right.$ 1)) $\mu_{H} \in S_{\mathrm{NK}_{1}}^{1}\left(\mathbf{U}^{(\beta)}\right)$ and $e^{-2 u} \eta_{2}+e^{-u} N\left(e^{-u} F_{2}\right) \mu_{H} \in S_{D_{0}}^{1}\left(\mathbf{U}^{(\beta)}\right)$, (5.1) is equivalent to

$$
\begin{aligned}
\lambda^{\mathcal{Q}}(\mathfrak{m}) & =\inf \left\{\mathcal{E}^{U^{(\beta)}}\left(f e^{u}, f e^{u}\right)-\int_{E} f^{2} \mathrm{~d}(\beta \mathfrak{m}+\bar{\nu})\right. \\
\quad & \left.-\int_{E} \int_{E} f(x) f(y)\left(e^{F(x, y)}-1\right) N(x, \mathrm{~d} y) \mu_{H}(\mathrm{~d} x) \mid f \in \mathcal{C}, \int_{E} f^{2} \mathrm{~d} \mathfrak{m}=1\right\} \\
= & \inf \left\{\mathcal{Q}(f, f) \mid f \in \mathcal{C}, \int_{E} f^{2} \mathrm{~d} \mathfrak{m}=1\right\}>0 .
\end{aligned}
$$


The proof of $\lambda^{\mathcal{Q}}\left(\bar{\mu}_{1}^{*}\right)>0 \Longleftrightarrow \lambda^{\mathcal{Q}}(\mathfrak{m})>0$ is the same as in the proof of $[26$, Propoition 5.1]. We omit it here.

For $p \geq 1$, put $F_{(p)}^{u}:=p F^{u}, G_{(p)}^{u}:=e^{F_{(p)}^{u}}-1$ and $V_{(p)}:=G_{(p)}^{u}-F_{(p)}^{u}+p F_{1}$. Define $\bar{\mu}^{p}:=\bar{\mu}_{1}^{p}-\bar{\mu}_{2}^{p}$ by

$$
\bar{\mu}_{1}^{p}:=N\left(V_{(p)}\right) \mu_{H}+p \mu_{1}+\frac{p^{2}}{2} \mu_{\langle u\rangle}^{c}, \quad \bar{\mu}_{2}^{p}:=N\left(p F_{2}\right) \mu_{H}+p \mu_{2} .
$$

Set

$$
\lambda^{\mathcal{Q}^{(p)}}\left(\bar{\mu}_{1}^{p}\right):=\inf \left\{\mathcal{Q}^{(p)}(f, f) \mid f \in \mathcal{C}, \int_{E} f^{2} \mathrm{~d} \bar{\mu}_{1}^{p}=1\right\}
$$

where $\mathcal{Q}^{(p)}$ is the quadratic form defined for $p u, p \mu$ and $p F$ as well as $\mathcal{Q}$ is defined for $u, \mu$ and $F$.

Proposition 5.2 (Super Gauge theorem). Suppose that $\bar{\mu}_{1}^{*} \in S_{\mathrm{NK}_{1}}^{1}(\mathbf{X})$, $\mu_{\langle u\rangle} \in S_{\mathrm{NK}_{\infty}}^{1}(\mathbf{X})$ and $\mu_{2}+N\left(F_{2}\right) \mu_{H} \in S_{D}^{1}(\mathbf{X})$ hold. Assume $\lambda^{\mathcal{Q}}\left(\bar{\mu}_{1}\right)>0$. Then there exists a $p_{0}>1$ sufficiently close to 1 such that $\lambda^{\mathcal{Q}^{(p)}}\left(\bar{\mu}_{1}^{p}\right)>0$ for any $p \in\left[1, p_{0}\right]$, hence we have $\sup _{x \in E} \mathbf{E}_{x}\left[e_{A}(\zeta)^{p}\right]<\infty$ for any $p \in\left[1, p_{0}\right]$ provided $\mu_{2}+N\left(F_{2}\right) \mu_{H} \in S_{D_{0}}^{1}(\mathbf{X})$.

Proof. We may assume $\mathfrak{m} \in S_{D_{0}}^{1}(\mathbf{X})$ and $\mathfrak{m}(E)<\infty$ in view of Lemma 5.1. Note that, for any $p$ sufficiently close to $1, p \mu_{1}+N\left(e^{p F_{1}^{u}}-p F_{1}^{u}-1+p F_{1}\right) \mu_{H}+$ $\frac{p^{2}}{2} \mu_{\langle u\rangle}^{c} \in S_{\mathrm{NK}_{1}}^{1}(\mathbf{X}), p^{2} \mu_{\langle u\rangle} \in S_{\mathrm{NK}_{\infty}}^{1}(\mathbf{X})$ and $p\left(\mu_{2}+N\left(F_{2}\right) \mu_{H}\right) \in S_{D}^{1}(\mathbf{X})$ as in the same way of the proof of Lemma 4.2(1). The rest of the proof is the same as in the proof of [26, Proposition 5.2]. We omit it.

LEMmA 5.2. Suppose that $\mu_{\langle u\rangle} \in S_{\mathrm{NK}_{\infty}}^{1}(\mathbf{X})$ holds. Then there exists $p_{0}>1$ sufficiently close to 1 such that $\sup _{x \in E} \mathbf{E}_{x}\left[U_{\zeta}^{p}\right]<\infty$ for any $p \in\left[1, p_{0}\right]$.

Proof. We may assume $\mathfrak{m} \in S_{D_{0}}^{1}(\mathbf{X})$ and $\mathfrak{m}(E)<\infty$ in view of Lemma 5.1. We observe the expression

$$
\begin{aligned}
U_{t} & =\operatorname{Exp}\left(M^{e^{U}-1}+M^{-u, c}\right)_{t} \\
& =\exp \left(M_{t}^{-u}-\int_{0}^{t} N\left(e^{U}-U-1\right)\left(X_{s}\right) \mathrm{d} H_{s}-\frac{1}{2}\left\langle M^{u, c}\right\rangle_{t}\right) \\
& =e^{u\left(X_{0}\right)-u\left(X_{t}\right)} \exp \left(-A_{t}^{N\left(e^{U}-U-1\right) \mu_{H}+\frac{1}{2} \mu_{\langle u\rangle}^{c}}+N_{t}^{u}\right) .
\end{aligned}
$$

The quadratic form $\mathcal{Q}$ associated to $A_{t}:=-A_{t}^{N\left(e^{U}-U-1\right) \mu_{H}+\frac{1}{2} \mu_{\langle u\rangle}^{c}}+N_{t}^{u}$ is given by

$$
\mathcal{Q}(f, g):=\mathcal{E}(f, g)+\mathcal{E}(f g, u)+\int_{E} f g N\left(e^{U}-U-1\right) \mathrm{d} \mu_{H}+\frac{1}{2} \int_{E} f g \mathrm{~d} \mu_{\langle u\rangle}^{c}
$$


for $f, g \in \mathcal{F}$. Note here that $N\left(e^{U}-U-1\right) \mu_{H}+\frac{1}{2} \mu_{\langle u\rangle}^{c} \in S_{\mathrm{NK}_{\infty}}^{1}(\mathbf{X}) \subset S_{D_{0}}^{1}(\mathbf{X})$. One can see that $\mathcal{Q}(f, g)=\mathcal{E}^{U}\left(f e^{u}, g e^{u}\right)$ for $f, g \in \mathcal{F}$. Then, we have

$$
\begin{aligned}
\lambda^{\mathcal{Q}}(\mathfrak{m}) & =\inf \left\{\mathcal{E}^{U}\left(f e^{u}, f e^{u}\right) \mid f \in \mathcal{F}, \int_{E} f^{2} \mathrm{~d} \mathfrak{m}=1\right\} \\
& \geq e^{-2\|u\|_{\infty}} \inf \left\{\mathcal{E}\left(f e^{u}, f e^{u}\right) \mid f \in \mathcal{F}, \int_{E} f^{2} \mathrm{~d} \mathfrak{m}=1\right\} \\
& =e^{-2\|u\|_{\infty}} \inf \left\{\mathcal{E}(f, f) \mid f \in \mathcal{F}, \int_{E} f^{2} e^{-2 u} \mathrm{~d} \mathfrak{m}=1\right\} \\
& \geq e^{-2\|u\|_{\infty}} \inf \left\{\mathcal{E}(f, f) \mid f \in \mathcal{F}, e^{2\|u\|_{\infty}} \int_{E} f^{2} \mathrm{~d} \mathfrak{m}=1\right\} \\
& =e^{-4\|u\|_{\infty}} \inf \left\{\mathcal{E}(f, f) \mid f \in \mathcal{F}, \int_{E} f^{2} \mathrm{~d} \mathfrak{m}=1\right\} \geq \frac{e^{-4\|u\|_{\infty}}}{\|R 1\|_{\infty}}>0 .
\end{aligned}
$$

One can confirm $\lambda^{\mathcal{Q}}\left(\bar{\mu}_{1}^{*}\right)>0$ for $\bar{\mu}_{1}^{*}:=N\left(e^{U}-U-1\right) \mu_{H}+\frac{1}{2} \mu_{\langle u\rangle}^{c}$ as shown in the proof of Proposition 5.1. Then we obtain the assertion in view of Proposition 5.2.

Corollary 5.1. Suppose that $\mu_{\langle u\rangle} \in S_{\mathrm{NK}_{\infty}}^{1}(\mathbf{X})$ holds. Then the following hold:

(1) For $\nu \in S_{D_{0}}^{1}(\mathbf{X}), e^{-2 u} \nu \in S_{D_{0}}^{1}(\mathbf{U})$.

(2) For $\nu \in S_{K_{\infty}}^{1}(\mathbf{X}), e^{-2 u} \nu \in S_{K_{\infty}}^{1}(\mathbf{U})$.

(3) For $\nu \in S_{\mathrm{CK}_{\infty}}^{1}(\mathbf{X}), e^{-2 u} \nu \in S_{\mathrm{CK}_{\infty}}^{1}(\mathbf{U})$.

(4) Suppose that $\mathrm{S}_{o}^{\nu} \supset \mathrm{S}_{o}^{\mu_{\langle u\rangle}^{c}+N\left(e^{U}-U-1\right) \mu_{H}}$ or there exists a measure $\eta \in$ $S_{\mathrm{NK}_{\infty}}^{1}(\mathbf{X})$ such that $\operatorname{supp}\left[A^{\eta}\right]=E$. Then, for $\nu \in S_{\mathrm{NK}_{\infty}}^{1}(\mathbf{X})$ we have $e^{-2 u} \nu \in S_{\mathrm{NK}_{\infty}}^{1}(\mathbf{U})$.

(5) For $\nu \in S_{\mathrm{NK}_{1}}^{1}(\mathbf{X})$ we have $e^{-2 u} \nu \in S_{\mathrm{NK}_{1}}^{1}(\mathbf{U})$.

Proof. As we proved in the previous lemma, there exists $p_{0}>1$ sufficiently close to 1 such that $\alpha(p):=\sup _{x \in E} \mathbf{E}_{x}\left[U_{\zeta}^{p}\right]<\infty$ for any $p \in\left[1, p_{0}\right]$. Put $q:=$ $p /(p-1)$ and $C(q):=q(q-1) \cdots(q-[q]+1)$ with $[q]:=\sup \{x \in \mathbb{N} \mid x \leq q\}$. Then, by Hölder's inequality and Lemma 2.2 in [17],

$$
R^{U}\left(e^{-2 u} \nu\right)(x)=\mathbf{E}_{x}^{U}\left[A_{\zeta}^{\nu}\right]=\mathbf{E}_{x}\left[U_{\zeta} A_{\zeta}^{\nu}\right] \leq \alpha(p)^{1 / p} C(q)^{1 / q} \sup _{x \in E} \mathbf{E}_{x}\left[A_{\zeta}^{\nu}\right] .
$$

This implies the assertions of (1), (2) and (3). Next, we prove (4) and (5). To prove them, we emphasize that (1) is needed. If $\sup _{x \in E} \mathbf{E}_{x}\left[A_{\tau_{D}}^{\nu}\right]<1$, then there exists $q \in] 1,2\left[\right.$ sufficiently close to 1 with $\sup _{x \in E} \mathbf{E}_{x}\left[A_{\tau_{D}}^{\nu}\right]<1 / q^{1 / q}$. Applying Hölder's inequality again

$$
\begin{aligned}
\mathbf{E}_{x}^{U}\left[A_{\tau_{D}}^{\nu}\right] & =\mathbf{E}_{x}\left[U_{\tau_{D}} A_{\tau_{D}}^{\nu}\right] \leq \mathbf{E}_{x}\left[U_{\tau_{D}}^{p}\right]^{\frac{1}{p}} \mathbf{E}_{x}\left[\left(A_{\tau_{D}}^{\nu}\right)^{q}\right]^{\frac{1}{q}} \\
& \leq \mathbf{E}_{x}\left[U_{\tau_{D}}^{p}\right]^{\frac{1}{p}} q^{\frac{1}{q}} \sup _{x \in E} \mathbf{E}_{x}\left[A_{\tau_{D}}^{\nu}\right]
\end{aligned}
$$


The rest of the proof is the same as in the proof of Lemma 4.1(6), (7).

Proof of Theorem 1.1. We may assume $\mathfrak{m} \in S_{D_{0}}^{1}(\mathbf{X})$ and $\mathfrak{m}(E)<\infty$ in view of Lemma 5.1. The gaugeability $\sup _{x \in E} \mathbf{E}_{x}\left[e_{A}(\zeta)\right]<\infty$ is equivalent to

$$
\sup _{x \in E} \mathbf{E}_{x}^{U}\left[e^{A_{\zeta}^{\bar{\nu}}+A_{\zeta}^{F}}\right]<\infty
$$

by (4.11). It suffices to prove that this is equivalent to (5.1). It is easy to see $\mathrm{S}_{o}^{\bar{\nu}_{1}} \supset \mathrm{S}_{o}^{\mu_{\langle u\rangle}^{c}+N\left(e^{U}-U-1\right) \mu_{H}}$. By Corollary 5.1(1), (5), we have $e^{-2 u} \mathfrak{m} \in S_{D_{0}}^{1}(\mathbf{U}), e^{-2 u}\left(\bar{\nu}_{1}+N\left(e^{U}\left(e^{F_{1}}-1\right)\right) \mu_{H}\right) \in S_{\mathrm{NK}_{1}}^{1}(\mathbf{U})$ and $e^{-2 u}\left(\bar{\nu}_{2}+\right.$ $\left.N\left(e^{U} F_{2}\right) \mu_{H}\right) \in S_{D_{0}}^{1}(\mathbf{U})$. Then we can apply Lemma $4.3(1) \Longleftrightarrow(4)$ so that

$$
\mathbf{E}_{x}^{U}\left[\int_{0}^{\zeta} e^{A_{s}^{\bar{\nu}}+A_{s}^{F}} \mathrm{~d} s\right]<\infty
$$

holds for some $x \in E$. The identity (5.2) with Theorem 4.1 for the function $g^{\beta \mathfrak{m}+\bar{\nu}+F}(x):=\mathbf{E}_{x}^{U^{(\beta)}}\left[\exp \left(\beta \zeta+A_{\zeta}^{\bar{\nu}}+A_{\zeta}^{F}\right)\right]$ under $\mathbf{U}^{(\beta)}$ yields the desired equivalence. The rest of the proof is the same as in the proof of Proposition 5.1.

\section{REMARK 5.1.}

(1) The quadratic form $(\check{\mathcal{Q}}, \check{\mathcal{F}})$ on $L^{2}(E ; g \mathfrak{m})$ obtained from the Dirichlet form $(\check{\mathcal{E}}, \check{\mathcal{F}})$ on $L^{2}(E ; g \mathfrak{m})$ associated to $(\check{\mathbf{X}}, g \mathfrak{m})$ by perturbations is given by $\check{\mathcal{Q}}(\varphi, \psi)=\check{\mathcal{E}}(\varphi, \psi)+\check{\mathcal{E}}(u, \varphi \psi)-\int_{E} \varphi \psi \mathrm{d} \mu-2 \int_{(E \times E) \backslash \operatorname{diag}} \varphi \otimes \psi\left(e^{F}-1\right) \mathrm{d} J$. The Feynman-Kac semigroup $\left(\check{Q}_{t}\right)_{t>0}$ associated with $(\check{\mathcal{Q}}, \check{\mathcal{F}})$ is given by

$$
\check{Q}_{t} f(x)=\check{\mathbf{E}}_{x}\left[\check{e}_{A}(t) f\left(\check{X}_{t}\right)\right]:=\mathbf{E}_{x}\left[e_{A}\left(\tau_{t}\right) f\left(X_{\tau_{t}}\right)\right]
$$

for $x \in E, f \in \mathcal{B}_{b}(E)$. Then the gauge function for the Feynman-Kac transform $\check{e}_{A}(t)$ obtained from $\left(\check{Q}_{t}\right)_{t>0}$ is given by

$$
\check{\mathbf{E}}_{x}\left[\check{e}_{A}(\check{\zeta})\right]=\mathbf{E}_{x}\left[e_{A}\left(\tau_{A_{\zeta}^{g \mathfrak{m}}}\right)\right]=\mathbf{E}_{x}\left[e_{A}(\zeta)\right], \quad x \in E,
$$

because $\tau_{A_{t}^{g \mathfrak{m}}}=t \wedge \zeta$. Now, applying Theorem 1.1 to $(\check{\mathbf{X}}, g \mathfrak{m})$, we see that

$$
\lambda^{\mathcal{Q}}\left(\bar{\mu}_{1}^{*}\right)=\lambda^{\check{\mathcal{Q}}}\left(\bar{\mu}_{1}^{*}\right):=\inf \left\{\check{\mathcal{Q}}(\varphi, \varphi) \mid \varphi \in \mathcal{C}, \int_{E} \varphi^{2} \mathrm{~d} \bar{\mu}_{1}^{*}=1\right\}>0
$$

is equivalent to $\sup _{x \in E} \mathbf{E}_{x}\left[e_{A}(\zeta)\right]<\infty$.

(2) In view of [26, Lemma 4.1](2), if (RSF) is satisfied for $\mathbf{X}$, the conclusion of Theorem 1.1 holds provided $\mu_{\langle u\rangle}+\mu_{1}+N\left(F_{1}\right) \mu_{H} \in S_{K_{\infty}}^{1}(\mathbf{X}), \mu_{2}+$ $N\left(F_{2}\right) \mu_{H} \in S_{D_{0}}^{1}(\mathbf{X})$.

The following corollary is an easy consequence of Theorem 1.1 through the same procedure as in the proof of Proposition 5.2.

Corollary 5.2 (Super Gauge Theorem). We have the following. 
(1) Suppose that $\bar{\mu}_{1}^{*} \in S_{\mathrm{NK}_{1}}^{1}(\mathbf{X}), \mu_{\langle u\rangle} \in S_{\mathrm{NK}_{\infty}}^{1}(\mathbf{X})$ and $\mu_{2}+N\left(F_{2}\right) \mu_{H} \in$ $S_{D_{0}}^{1}(\mathbf{X})$ hold. If $\sup _{x \in E} \mathbf{E}_{x}\left[e_{A}(\zeta)\right]<\infty$, then there exists a $p_{0}>1$ sufficiently close to 1 such that $\sup _{x \in E} \mathbf{E}_{x}\left[e_{A}(\zeta)^{p}\right]<\infty$ for any $p \in\left[1, p_{0}\right]$.

(2) Suppose that $N\left(e^{U}\left(e^{F_{1}}-1\right)\right) \mu_{H} \in S_{\mathrm{NK}_{1}}^{1}(\mathbf{X}), \quad \mu_{\langle u\rangle} \in S_{\mathrm{NK}_{\infty}}^{1}(\mathbf{X})$ and $N\left(F_{2}\right) \mu_{H} \in S_{D_{0}}^{1}(\mathbf{X})$ hold. Then there exists a $p_{0}>1$ sufficiently close to 1 such that $\sup _{x \in E} \mathbf{E}_{x}\left[\left(Y_{\zeta}^{1}\right)^{p}\right]<\infty$ for any $p \in\left[1, p_{0}\right]$. In particular, the assertions in Corollary 5.1 remain valid by replacing the transformed process $\mathbf{U}$ by $\mathbf{Y}^{1}$ or $\mathbf{Z}$.

Proof. (1): By Theorem 1.1, the gaugeability $\sup _{x \in E} \mathbf{E}_{x}\left[e_{A}(\zeta)\right]<\infty$ is equivalent to $\lambda^{\mathcal{Q}}\left(\bar{\mu}_{1}\right)>0$ without assuming $\mathfrak{m} \in S_{D_{0}}^{1}(\mathbf{X})$ and $\mathfrak{m}(E)<\infty$. In the same way of the proof of Proposition 5.2, we have that there exists a $\left.p_{0} \in\right] 1,+\infty\left[\right.$ sufficiently close to 1 such that $\lambda^{\mathcal{Q}^{(p)}}\left(\bar{\mu}_{1}^{(p)}\right)>0$ for any $p \in\left[1, p_{0}\right]$, equivalently $\sup _{x \in E} \mathbf{E}_{x}\left[e_{A}(\zeta)^{p}\right]<\infty$ holds for any $p \in\left[1, p_{0}\right]$ by Theorem 1.1.

(2): The proof of (2) is easy in view of the following expressions for $Y_{t}^{1}$ and $Z_{t}$ :

$$
\begin{aligned}
Y_{t}^{1} & =e^{u\left(X_{0}\right)-u\left(X_{t}\right)} e_{A-A^{\bar{\mu}^{*}}+A^{F_{2}}(t),} \\
Z_{t} & =e^{u\left(X_{0}\right)-u\left(X_{t}\right)} e_{A-A^{\bar{\mu}^{*}}}(t) .
\end{aligned}
$$

Corollary 5.3. Suppose that $\bar{\mu}_{1}^{*} \in S_{\mathrm{NK}_{1}}^{1}(\mathbf{X}), \mu_{\langle u\rangle} \in S_{\mathrm{NK}_{\infty}}^{1}(\mathbf{X})$ and $\mu_{2}+$ $N\left(F_{2}\right) \mu_{H} \in S_{D_{0}}^{1}(\mathbf{X})$ hold. Then $\inf _{x \in E} \mathbf{E}_{x}\left[e_{A}(\zeta)\right]>0$ holds.

Proof. Note that $e^{-2 u} \bar{\nu}_{2} \in S_{D_{0}}^{1}(\mathbf{U})$ and $e^{-2 u} N\left(e^{U} F_{2}\right) \mu_{H} \in S_{D_{0}}^{1}(\mathbf{U})$ in view of Corollary 5.1(1). Then by Jensen's inequality, we have

$$
\begin{aligned}
\inf _{x \in E} \mathbf{E}_{x}^{U}\left[\exp \left(A_{\zeta}^{\bar{\nu}}+A_{\zeta}^{F}\right)\right] & \geq \inf _{x \in E} \mathbf{E}_{x}^{U}\left[\exp \left(-A_{\zeta}^{\bar{\nu}_{2}}-A_{\zeta}^{F_{2}}\right)\right] \\
& \geq \exp \left(-\sup _{x \in E} \mathbf{E}_{x}^{U}\left[A_{\zeta}^{\bar{\nu}_{2}}+A_{\zeta}^{F_{2}}\right]\right)>0 .
\end{aligned}
$$

Applying (4.11), we now obtain

$$
\inf _{x \in E} \mathbf{E}_{x}\left[e_{A}(\zeta)\right] \geq \inf _{x \in E} \mathbf{E}_{x}^{U}\left[\exp \left(A_{\zeta}^{\bar{\nu}}+A_{\zeta}^{F}\right)\right] e^{-2\|u\|_{\infty}}>0 .
$$

Theorem 5.1 (Gauge theorem). Assume $\bar{\mu}_{1}^{*} \in S_{\mathrm{NK}_{1}}^{1}(\mathbf{X}), \mu_{\langle u\rangle} \in S_{\mathrm{NK}_{\infty}}^{1}(\mathbf{X})$ and $\mu_{2}+N\left(F_{2}\right) \mu_{H} \in S_{1}(\mathbf{X})$. Then the following are equivalent to each other.

(1) There exists $x \in E$ such that $\mathbf{E}_{x}\left[e_{A}(\zeta)\right]<\infty$.

(2) $\sup _{x \in E} \mathbf{E}_{x}\left[e_{A}(\zeta)\right]<\infty$.

Proof. The proof is quite similar to the proofs of [4, Theorem 2.13], [10, Theorem 2.6]. By (4.11), we see

$$
e^{-2\|u\|_{\infty}} \mathbf{E}_{x}^{U}\left[\exp \left(A_{\zeta}^{\bar{\nu}}+A_{\zeta}^{F}\right)\right] \leq g_{A}(x) \leq e^{2\|u\|_{\infty}} \mathbf{E}_{x}^{U}\left[\exp \left(A_{\zeta}^{\bar{\nu}}+A_{\zeta}^{F}\right)\right] .
$$

So it suffices to prove $g^{U}(x):=\mathbf{E}_{x}^{U}\left[\exp \left(A_{\zeta}^{\bar{\nu}}+A_{\zeta}^{F}\right)\right]$ satisfies $\sup _{x \in E} g^{U}(x)<\infty$ or $g^{U} \equiv \infty$. The fine continuity of $x \mapsto g^{U}(x)$ and the absorbing property of 
the set $O:=\left\{g^{U}<\infty\right\}$ can be proved in a similar way as in [4], [10]. So, it suffices to confirm that there are $N>0$ and $C>0$ such that

$$
O=\left\{g^{U}<\infty\right\}=\left\{g^{U} \leq C(1+N)\right\} .
$$

Then $O$ is finely open and finely closed. Since $E$ is connected with respect to the fine topology under $(\mathbf{I})$ and $(\mathbf{A C})$, we have $O=E$ or $O=\emptyset$, which means the assertion. Next, we prove $e^{-2 u} \bar{\nu}_{1}+e^{-u} N\left(e^{-u}\left(e^{F_{1}}-1\right)\right) \mu_{H} \in S_{\mathrm{NK}_{1}}^{1}$ (U). We know $\bar{\nu}_{1}+N\left(e^{U}\left(e^{F_{1}}-1\right)\right) \mu_{H} \in S_{\mathrm{NK}_{1}}^{1}(\mathbf{X})$ for $\bar{\nu}_{1}:=\mu_{1}+N\left(e^{U}-U-1\right) \mu_{H}+$ $\frac{1}{2} \mu_{\langle u\rangle}^{c}$. Since $\bar{\nu}_{1}+N\left(e^{U}\left(e^{F_{1}}-1\right)\right) \mu_{H} \geq \frac{1}{2} \mu_{\langle u\rangle}^{c}+N\left(e^{U}-U-1\right) \mu_{H}$, we have $\mathrm{S}_{o}^{\bar{\nu}_{1}+N\left(e^{U}\left(e^{F_{1}}-1\right)\right) \mu_{H}} \supset \mathrm{S}_{o}^{\frac{1}{2} \mu_{\langle u\rangle}^{c}+N\left(e^{U}-U-1\right) \mu_{H}}=\mathrm{S}_{O}^{\mu_{\langle u\rangle}^{c}+N\left(e^{U}-U-1\right) \mu_{H}}$. We can apply Corollary 5.1(5) so that $e^{-2 u} \bar{\nu}_{1}+e^{-u} N\left(e^{-u}\left(e^{F_{1}}-1\right)\right) \mu_{H} \in S_{\mathrm{NK}_{1}}^{1}$ (U). The proof of $e^{-2 u} \bar{\nu}_{2}+e^{-u} N\left(e^{-u} F_{2}\right) \mu_{H} \in S_{1}(\mathbf{U})$ is easy. The rest of the proof is the same as in the proof of Theorem 4.1 .

REMARK 5.2. Theorem 5.1 is not covered by [22, (4.5) Theorem] due to the existence of $u$.

In the rest of this paper, we use the notation $\tau_{D}$ as the first exit time of $\mathbf{X}$ out of $D$ in $E$, that is, $\tau_{D}=\inf \left\{t>0: X_{t} \notin D\right\}$.

The following is a trivial consequence of Theorem 1.1.

COROllary 5.4. Let $\mathbf{X}$ be an $\mathfrak{m}$-symmetric (not necessarily irreducible) Feller process on $E$. Let $D$ be a connected open subset of $E$ and $\mathbf{X}_{D}$ the part process of $\mathbf{X}$ on $D$. Assume that (RSF) is satisfied for $\mathbf{X}$ and take $u \in\left(\mathcal{F}_{D}\right)_{\text {loc }} \cap C\left(D_{\partial}\right)$. Then $\lambda_{D}^{\mathcal{Q}}\left(\bar{\mu}_{1}\right)>0$ if and only if $\sup _{x \in D} \mathbf{E}_{x}\left[e_{A}\left(\tau_{D}\right)\right]<\infty$ provided $\bar{\mu}_{1}^{*} \in S_{\mathrm{CK}_{1}}^{1}\left(\mathbf{X}_{D}\right), \mu_{\langle u\rangle} \in S_{K_{\infty}}^{1}\left(\mathbf{X}_{D}\right)$ and $\mu_{2}+N\left(F_{2}\right) \mu_{H} \in S_{D_{0}}^{1}\left(\mathbf{X}_{D}\right)$. Here $\lambda_{D}^{\mathcal{Q}}\left(\bar{\mu}_{1}\right):=\inf \left\{\mathcal{Q}(f, f) \mid f \in \mathcal{C}_{D}, \int_{D} f^{2} \mathrm{~d} \bar{\mu}_{1}=1\right\}$.

Proof. Under the condition, $\mathbf{X}_{D}$ satisfies (RSF) by [26, Lemma 5.3], hence $S_{\mathrm{CK} \infty}^{1}\left(\mathbf{X}_{D}\right)=S_{K_{\infty}}^{1}\left(\mathbf{X}_{D}\right)$ from [26, Lemma 4.1]. Moreover, (I) holds for $\mathbf{X}_{D}$ because of the connectedness of $D$.

\section{Semi-conditional gaugeability and subcriticality}

The following definitions of Green-tight measures of Kato class are due to Chen [4]. Let $\mathrm{d}:=\{(x, z) \in E \times E \mid R(x, z)=0$ or $\infty\}$ and $E^{z}:=\{x \in E \mid 0<$ $R(x, z)<\infty\}$.

First, we show the following lemma, which was not stated in [26].

LEMMA 6.1. Under the conditions (I) and (AC), $R(x, y)>0$ holds for any $x, y \in E$, in particular, $\mathrm{d}=\{(x, y) \in E \times E \mid R(x, y)=\infty\}$.

Proof. We may assume $E \neq \emptyset$. Otherwise, the statement is true. Fix $x \in E$. First we note that $\alpha R_{\alpha}(R(x, \cdot))(y) \leq R(x, y)$ and $\alpha \mapsto \alpha R_{\alpha}(R(x, \cdot))(y)$ is increasing for $y \in E$. We set $\bar{R}(x, y):=\lim _{\alpha \rightarrow \infty} \alpha R_{\alpha}(R(x, \cdot))(y) \leq R(x, y)$ 
and $D_{x}:=\{y \in E \mid \bar{R}(x, y)>0\}$. Note that $\bar{R}(x, y)=R(x, y)$ m-a.e. $y \in E$. Then $\mathbf{1}_{D_{x}}$ is an excessive function of $\mathbf{X}$. Indeed,

$$
\begin{aligned}
\lim _{\alpha \rightarrow \infty} \alpha R_{\alpha} \mathbf{1}_{D_{x}}(y) & =\lim _{\alpha \rightarrow \infty} \lim _{n \rightarrow \infty} \alpha R_{\alpha}(n \bar{R}(x, \cdot) \wedge 1)(y) \\
& =\lim _{n \rightarrow \infty} \lim _{\alpha \rightarrow \infty} \alpha R_{\alpha}(n \bar{R}(x, \cdot) \wedge 1)(y) \\
& =\lim _{n \rightarrow \infty}(n \bar{R}(x, \cdot) \wedge 1)(y)=\mathbf{1}_{D_{x}}(y) .
\end{aligned}
$$

Thus, we have that $D_{x}$ is Borel measurable, and finely open and finely closed. Under $(\mathbf{I})$ and $(\mathbf{A C})$, the fine topology of $\mathbf{X}$ is connected. Then we obtain $D_{x}=\emptyset$ or $D_{x}=E$. The case $D_{x}=\emptyset$ yields $\bar{R}(x, y)=0$ for all $x, y \in E$, which implies $R f(x)=\int_{E} R(x, y) f(y) \mathfrak{m}(\mathrm{d} y)=\int_{E} \bar{R}(x, y) f(y) \mathfrak{m}(\mathrm{d} y)=0$ for any $f \in$ $L^{2}(E ; \mathfrak{m})$. Consequently $L^{2}(E ; \mathfrak{m})=\{0\}$, hence $C_{0}(E)=\{0\}$. Thus, we get $E=\emptyset$ contradicting $E \neq \emptyset$. Therefore, we obtain $D_{x}=E$, hence $R(x, y) \geq$ $\bar{R}(x, y)>0$ for all $x, y \in E$.

Definition 6.1 (Semi-conditionally Green-bounded, semi-conditionally Green tight Kato class measures in the sense of Chen). Let $\nu \in S_{1}(\mathbf{X})$ and denote by $R_{\alpha}^{z}(x, y)$ the $\alpha$-order Green function of Doob's $R(\cdot, z)$-transformed process $\mathbf{X}^{z}$ of $\mathbf{X}$ for $\alpha \geq 0$ defined by

$$
R_{\alpha}^{z}(x, y):=\frac{R_{\alpha}(x, y) R(y, z)}{R(x, z)}, \quad x, y \in E^{z} \text { with }(x, y) \in(E \times E) \backslash \mathrm{d},
$$

where $\mathrm{d}:=\{(x, y) \mid R(x, y)=0$ or $+\infty\}$ and $E^{z}:=\{x \in E \mid(x, z) \in(E \times E) \backslash \mathrm{d}\}$. We write $R^{z}(x, y):=R_{0}^{z}(x, y)$ for $\alpha=0$.

(1) $\nu$ is said to be semi-conditionally Green-bounded if $\nu \in \bigcap_{z \in E} S_{D_{0}}^{1}\left(\mathbf{X}^{z}\right) . \nu$ is said to be conditionally Green-bounded if

$$
\sup _{(x, z) \in(E \times E) \backslash \mathrm{d}} \int_{E^{z}} R^{z}(x, y) \nu(\mathrm{d} y)<\infty .
$$

(2) $\nu$ is said to be a semi-conditionally Green-tight measure of Kato class in the sense of Chen if $\nu \in \bigcap_{z \in E} S_{\mathrm{CK}_{\infty}}^{1}\left(\mathbf{X}^{z}\right) . \nu$ is said to be a conditionally Green-tight measure of Kato class in the sense of Chen if for any $\varepsilon>0$ there exist a Borel set $K=K(\varepsilon) \subset E$ of finite $\nu$-measure and a constant $\delta>0$ such that for all $\nu$-measurable set $B \subset K$ with $\nu(B)<\delta$,

$$
\sup _{(x, z) \in(E \times E) \backslash \mathrm{d}} \int_{B \cup K^{c}} R^{z}(x, y) \nu(\mathrm{d} y)<\varepsilon .
$$

(3) $\nu$ is said to be a semi-conditionally semi-Green-tight measure of extended Kato class in the sense of Chen if $\nu \in \bigcap_{z \in E} S_{\mathrm{CK}_{1}}^{1}\left(\mathbf{X}^{z}\right) . \nu$ is said to be a conditionally semi-Green-tight measure of extended Kato class in the sense of Chen if there exist a Borel set $K \subset E$ of finite $\nu$-measure and a 
constant $\delta>0$ such that for all $\nu$-measurable set $B \subset K$ with $\nu(B)<\delta$,

$$
\sup _{(x, z) \in(E \times E) \backslash \mathrm{d}} \int_{B \cup K^{c}} R^{z}(x, y) \nu(\mathrm{d} y)<1 .
$$

Let us denote by $S_{\mathrm{CS}_{\infty}}^{1}(\mathbf{X})$ (resp. $\left.S_{\mathrm{CS}_{1}}^{1}(\mathbf{X}), S_{\mathrm{DS}_{0}}^{1}(\mathbf{X})\right)$ the family of conditionally Green-tight measures of Kato class in the sense of Chen (resp. the family of conditionally semi-Green-tight measures of extended Kato class in the sense of Chen, the family of conditionally Green-bounded measures) and semi$S_{\mathrm{CS}_{\infty}}^{1}(\mathbf{X}):=\bigcap_{z \in E} S_{\mathrm{CK}_{\infty}}^{1}\left(\mathbf{X}^{z}\right)$ (resp. semi- $S_{\mathrm{CS}_{1}}^{1}(\mathbf{X}):=\bigcap_{z \in E} S_{\mathrm{CK}_{1}}^{1}\left(\mathbf{X}^{z}\right)$, semi$\left.S_{\mathrm{DS}_{0}}^{1}(\mathbf{X}):=\bigcap_{z \in E} S_{D_{0}}^{1}\left(\mathbf{X}^{z}\right)\right)$ the family of semi-conditionally Green-tight measures of Kato class in the sense of Chen (resp. the family of semi-conditionally semi-Green-tight measures of extended Kato class in the sense of Chen, the family of semi-conditionally Green-bounded measures). Clearly, $S_{\mathrm{CS}_{\infty}}^{1}(\mathbf{X}) \subset$ $S_{\mathrm{CS}_{1}}^{1}(\mathbf{X}) \subset S_{\mathrm{DS}_{0}}^{1}(\mathbf{X}), S_{\mathrm{CS}_{\infty}}^{1}(\mathbf{X}) \subset \operatorname{semi}-S_{\mathrm{CS}_{\infty}}^{1}(\mathbf{X}), S_{\mathrm{CS}_{1}}^{1}(\mathbf{X}) \subset \operatorname{semi}-S_{\mathrm{CS}_{1}}^{1}(\mathbf{X})$ and $S_{\mathrm{DS}_{0}}^{1}(\mathbf{X}) \subset \operatorname{semi}-S_{\mathrm{DS}_{0}}^{1}(\mathbf{X})$.

It is known $S_{\mathrm{CS}_{\infty}}^{1}(\mathbf{X}) \subset S_{\mathrm{CK}_{\infty}}^{1}(\mathbf{X})$ and $S_{\mathrm{CS}_{1}}^{1}(\mathbf{X}) \subset S_{\mathrm{CK}_{1}}^{1}(\mathbf{X})([4$, the remark after Definition 3.1] and [9, Proposition 3.1 and Corollary 3.1]) and $S_{\mathrm{DS}_{0}}^{1}(\mathbf{X}) \subset$ $S_{D_{0}}^{1}(\mathbf{X})$ can be similarly proved along the proof of $[9$, Proposition 3.1 and Corollary 3.1]. The converse inclusions $S_{\mathrm{CK}_{\infty}}^{1}(\mathbf{X}) \subset S_{\mathrm{CS}_{\infty}}^{1}(\mathbf{X}), \frac{1}{2 c} \cdot S_{\mathrm{CK}_{1}}^{1}(\mathbf{X}) \subset$ $S_{\mathrm{CS}_{1}}^{1}(\mathbf{X}) \quad$ and $\quad S_{D_{0}}^{1}(\mathbf{X}) \subset S_{\mathrm{DS}_{0}}^{1}(\mathbf{X}), \quad$ hence $S_{\mathrm{CK}_{\infty}}^{1}(\mathbf{X})=$ $S_{\mathrm{CS}_{\infty}}^{1}(\mathbf{X}), \frac{1}{2 c} \cdot S_{\mathrm{CK}_{1}}^{1}(\mathbf{X}) \subset S_{\mathrm{CS}_{1}}^{1}(\mathbf{X}) \subset S_{\mathrm{CK}_{1}}^{1}(\mathbf{X})$ and $S_{D_{0}}^{1}(\mathbf{X})=S_{\mathrm{DS}_{0}}^{1}(\mathbf{X})$ hold under the $3 G$-inequality: $R^{z}(x, y) \leq c(R(x, y)+R(y, z))$. Here $\frac{1}{2 c} \cdot S_{\mathrm{CK}_{1}}^{1}(\mathbf{X}):=$ $\left\{\frac{1}{2 c} \nu \mid \nu \in S_{\mathrm{CK}_{1}}^{1}(\mathbf{X})\right\}$. Moreover, $S_{\mathrm{CS}_{1}}^{1}(\mathbf{X}) \subset S_{\mathrm{DS}_{0}}^{1}(\mathbf{X})$ holds by [4, Proposition 3.2]. Note that the Borel set $K$ of finite measure appeared in Definition 6.1 can be taken to be compact (cf. the argument after Definition 4.1).

Definition 6.2. Let $F$ be a symmetric bounded function on $E \times E$ vanishing on the diagonal set such that $F$ can be extended to $(E \times\{\partial\}) \cup(\{\partial\} \times\{\partial\})$ with $F(x, \partial)=F(\partial, \partial)=0$ for $x \in E$.

(1) $F$ is said to be in the class $J_{\mathrm{DS}_{0}}^{1}(\mathbf{X})$ if $N(|F|) \mu_{H} \in S_{\mathrm{DS}_{0}}^{1}(\mathbf{X}) . F$ is said to be in the class $A_{\mathrm{DS}_{0}}^{1}(\mathbf{X})$ if

$$
\sup _{(x, z) \in(E \times E) \backslash \mathrm{d}} \int_{E \times E} \frac{R(x, y)|F(y, w)| R(w, z)}{R(x, z)} N(y, \mathrm{~d} w) \mu_{H}(\mathrm{~d} y)<\infty .
$$

(2) $F$ is said to be in the class $J_{\mathrm{CK}_{\infty}}^{1}(\mathbf{X})\left(\operatorname{resp} . J_{\mathrm{CS}_{\infty}}^{1}(\mathbf{X})\right)$ if $N(|F|) \mu_{H} \in$ $S_{\mathrm{CK}_{\infty}}^{1}(\mathbf{X})$ (resp. $\left.N(|F|) \mu_{H} \in S_{\mathrm{CS}_{\infty}}^{1}(\mathbf{X})\right) . \quad F$ is said to be in the class $A_{\mathrm{CS}_{\infty}}^{1}(\mathbf{X})$ if for any $\varepsilon>0$, there exist a Borel set $K=K(\varepsilon) \subset E$ of finite $N(|F|) \mu_{H}$-measure and a constant $\delta>0$ such that for all measurable set $B \subset K$ with $\int_{B} N(|F|) \mathrm{d} \mu_{H}<\delta$,

$$
\sup _{(x, z) \in(E \times E) \backslash \mathrm{d}} \int_{\{(K \backslash B) \times(K \backslash B)\}^{c}} \frac{R(x, y)|F(y, w)| R(w, z)}{R(x, z)} N(y, \mathrm{~d} w) \mu_{H}(\mathrm{~d} y)<\varepsilon .
$$


(3) $F$ is said to be in the class $J_{\mathrm{CK}_{1}}^{1}(\mathbf{X})$ (resp. $\left.J_{\mathrm{CS}_{1}}^{1}(\mathbf{X})\right)$ if $N(|F|) \mu_{H} \in$ $S_{\mathrm{CK}_{1}}^{1}(\mathbf{X})$ (resp. $\left.N(|F|) \mu_{H} \in S_{\mathrm{CS}_{1}}^{1}(\mathbf{X})\right) . \quad F$ is said to be in the class $A_{\mathrm{CS}_{1}}^{1}(\mathbf{X})$ if there exist a Borel set $K \subset E$ of finite $N(|F|) \mu_{H}$-measure and a constant $\delta>0$ such that for all measurable set $B \subset K$ with $\int_{B} N(|F|) \mathrm{d} \mu_{H}<\delta$

$$
\sup _{(x, z) \in(E \times E) \backslash \mathrm{d}} \int_{\{(K \backslash B) \times(K \backslash B)\}^{c}} \frac{R(x, y)|F(y, w)| R(w, z)}{R(x, z)} N(y, \mathrm{~d} w) \mu_{H}(\mathrm{~d} y)<1 .
$$

By a similar proof as that for [10, Corollary 3.2], we have $A_{\mathrm{CS}_{\infty}}^{1}(\mathbf{X}) \subset$ $J_{\mathrm{CK}_{\infty}}^{1}(\mathbf{X}), A_{\mathrm{CS}_{1}}^{1}(\mathbf{X}) \subset J_{\mathrm{CK}_{1}}^{1}(\mathbf{X})$ and $A_{\mathrm{DS}_{0}}^{1}(\mathbf{X}) \subset J_{D_{0}}^{1}(\mathbf{X})$. Moreover, according to the same way of the proof of Proposition 3.2 in [4], we see $A_{\mathrm{CS}_{\infty}}^{1}(\mathbf{X}) \subset$ $A_{\mathrm{CS}_{1}}^{1}(\mathbf{X}) \subset A_{\mathrm{DS}_{0}}^{1}(\mathbf{X})$.

REMARK 6.1. The definition for $A_{\infty}(\mathbf{X})$ in [10, Definition 3.2] is incorrect. We follow the definition as in [4, Definition 3.4]. So [26, Definition 6.2(2)(3)] should be corrected as in Definition 6.2(2), (3) above.

Definition 6.3. Let $F$ be a symmetric bounded function on $E \times E$ noted in Definition 6.2 above and $\nu \in S_{1}(\mathbf{X})$.

(1) $(\nu, F)$ is said to be in the class $B_{\mathrm{DS}_{0}}^{1}(\mathbf{X})$ if $\nu \in S_{\mathrm{DS}_{0}}^{1}(\mathbf{X})$ and $F \in A_{\mathrm{DS}_{0}}^{1}(\mathbf{X})$.

(2) $(\nu, F)$ is said to be in the class $B_{\mathrm{CS}_{\infty}}^{1}(\mathbf{X})$ if $\nu \in S_{\mathrm{CS}_{\infty}}^{1}(\mathbf{X})$ and $F \in$ $A_{\mathrm{CS}}^{1}(\mathbf{X})$.

(3) $(\nu, F)$ is said to be in the class $B_{\mathrm{CS}_{1}}^{1}(\mathbf{X})$ if there exist a Borel set $K \subset E$ of finite $\nu+N(|F|) \mu_{H}$-measure and a constant $\delta>0$ such that for all measurable set $B \subset K$ with $\nu(B)+\int_{B} N(|F|) \mathrm{d} \mu_{H}<\delta$,

$$
\begin{aligned}
& \sup _{(x, z) \in(E \times E) \backslash \mathrm{d}}\left(\int_{B \cup K^{c}} R^{z}(x, y) \nu(\mathrm{d} y)\right. \\
& \left.+\int_{\{(K \backslash B) \times(K \backslash B)\}^{c}} \frac{R(x, y)|F(y, w)| R(w, z)}{R(x, z)} N(y, \mathrm{~d} w) \mu_{H}(\mathrm{~d} y)\right)<1 .
\end{aligned}
$$

Clearly, $(\nu, F) \in B_{\mathrm{CS}_{1}}^{1}(\mathbf{X})$ implies $\nu \in S_{\mathrm{CS}_{1}}^{1}(\mathbf{X})$ and $F \in A_{\mathrm{CS}_{1}}^{1}(\mathbf{X})$. In particular, we have $B_{\mathrm{CS}_{\infty}}^{1}(\mathbf{X}) \subset B_{\mathrm{CS}_{1}}^{1}(\mathbf{X}) \subset B_{\mathrm{DS}_{0}}^{1}(\mathbf{X})$.

Note that the Borel set $K$ of finite measure appeared in Definitions 6.2 and 6.3 can be taken to be compact (cf. the argument after Definition 4.1).

The following lemma is needed in the proof of Theorem 6.1.

Lemma 6.2. Take $\nu \in S_{1}(\mathbf{X})$ and $F \in J_{1}(\mathbf{X})$. Let $g \in L^{1}\left(E ; \nu+N(|F|) \mu_{H}\right)$ be a $\left(\nu+N(|F|) \mu_{H}\right)$-a.e. strictly positive bounded function. Then the following are equivalent each other.

(1) The following are equivalent.

(a) $(\nu, F) \in B_{\mathrm{CS}_{\infty}}^{1}(\mathbf{X})$. 
(b) $(\nu, F) \in B_{\mathrm{DS}_{0}}^{1}(\mathbf{X})$ and for every decreasing sequence of Borel sets $\left\{D_{n}\right\}$ with empty intersection

$$
\begin{aligned}
& \lim _{n \rightarrow \infty} \sup _{(x, z) \in(E \times E) \backslash \mathrm{d}}\left(\int_{D_{n}} R^{z}(x, y) \nu(\mathrm{d} y)\right. \\
& \left.\quad+\int_{\left(D_{n} \times E\right) \cup\left(E \times D_{n}\right)} \frac{R(x, y)|F(y, w)| R(w, z)}{R(x, z)} N(y, \mathrm{~d} w) \mu_{H}(\mathrm{~d} y)\right)=0 .
\end{aligned}
$$

(c) For any $\varepsilon>0$ there exist a Borel subset $K=K(\varepsilon)$ of $E$ and a constant $\delta>0$ such that for all measurable set $B \subset K$ with $\int_{B} g \mathrm{~d}(\nu+$ $\left.N(|F|) \mu_{H}\right)<\delta$,

$$
\begin{aligned}
& \sup _{(x, z) \in(E \times E) \backslash \mathrm{d}}\left(\int_{B \cup K^{c}} R^{z}(x, y) \nu(\mathrm{d} y)\right. \\
& \left.+\int_{\{(K \backslash B) \times(K \backslash B)\}^{c}} \frac{R(x, y)|F(y, w)| R(w, z)}{R(x, z)} N(y, \mathrm{~d} w) \mu_{H}(\mathrm{~d} y)\right)=0 .
\end{aligned}
$$

(2) The following are equivalent.

(a) $(\nu, F) \in B_{\mathrm{CS}_{1}}^{1}(\mathbf{X})$.

(b) $(\nu, F) \in B_{\mathrm{DS}_{0}}^{1}(\mathbf{X})$ and for every decreasing sequence of Borel sets $\left\{D_{n}\right\}$ with empty intersection

$$
\begin{aligned}
& \lim _{n \rightarrow \infty} \sup _{(x, z) \in(E \times E) \backslash \mathrm{d}}\left(\int_{D_{n}} R^{z}(x, y) \nu(\mathrm{d} y)\right. \\
& \left.\quad+\int_{\left(D_{n} \times E\right) \cup\left(E \times D_{n}\right)} \frac{R(x, y)|F(y, w)| R(w, z)}{R(x, z)} N(y, \mathrm{~d} w) \mu_{H}(\mathrm{~d} y)\right)<1 .
\end{aligned}
$$

(c) There exist a Borel subset $K$ of $E$ and a constant $\delta>0$ such that for all measurable set $B \subset K$ with $\int_{B} g \mathrm{~d}\left(\nu+N(|F|) \mu_{H}\right)<\delta$, (6.1) holds.

Proof. The proof of Lemma 6.2 is similar to the proof of [26, Lemma 4.2] (see also [4, Propositions 2.2 and 2.4]). So we omit the detail.

Let $\bar{\mu}_{1}^{*} \in S_{\mathrm{EK}}^{1}(\mathbf{X}), \mu_{\langle u\rangle} \in S_{K}^{1}(\mathbf{X})$ and $\mu_{2}+N\left(F_{2}\right) \mu_{H} \in S_{1}(\mathbf{X})$. In the same way of Lemma 4.1 in [19], for $\alpha \geq 0$, we can construct a symmetric $\alpha$-order resolvent kernel $R_{\alpha}^{A}(x, y)$ of the Feynman-Kac semigroup $P_{t}^{A} f(x):=$ $\mathbf{E}_{x}\left[e_{A}(t) f\left(X_{t}\right)\right]$, which is defined for all $x, y \in E$ (possibly infinite), $\alpha$-excessive with respect to $\left(P_{t}^{A}\right)_{t \geq 0}$ in $x$ (and in $y$ ), and satisfies a resolvent equation like as in $[19,(4.2 .12)]$.

We need the following lemmas:

LEMma 6.3 (cf. [26, Lemma 6.1]). Suppose that $\bar{\mu}_{1}^{*} \in S_{\mathrm{EK}}^{1}(\mathbf{X}), \mu_{\langle u\rangle} \in$ $S_{K}^{1}(\mathbf{X})$ and $\mu_{2}+N\left(F_{2}\right) \mu_{H} \in S_{1}(\mathbf{X})$ hold. Then for $\alpha \geq 0$ and $x \in E$, $y \mapsto R_{\alpha}^{A}(x, y)$ is finely continuous with respect to $\mathbf{X}$.

Proof. The proof is the quite same as that of [26, Lemma 6.1] under the condition above. So we omit it. 
Lemma 6.4 (cf. [26, Lemma 6.2]). Assume that $\bar{\mu}_{1}^{*} \in$ semi- $_{\mathrm{CS}_{1}}^{1}(\mathbf{X}), \mu_{\langle u\rangle} \in$ semi- $S_{\mathrm{CS}_{\infty}}^{1}(\mathbf{X})$ and $\mu_{2}+N\left(F_{2}\right) \mu_{H} \in$ semi- $S_{\mathrm{DS}_{0}}^{1}(\mathbf{X})$. Then for each $y \in E$, $E^{y} \ni x \mapsto \mathbf{E}_{x}^{y}\left[e_{A}\left(\zeta^{y}\right)\right]$ is finely lower semi continuous with respect to $\mathbf{X}^{y}$.

Proof. The proof is the quite same as that of [26, Lemma 6.2] under the condition above. So we omit it.

The following theorems and lemma can be proved with slight modifications as those in [4].

Theorem 6.1 (Conditional Gauge Theorem I, cf. [4, Theorem 3.8], [10, Theorem 3.5]). Suppose $\left(\nu_{1}, e^{F_{1}}-1\right) \in B_{\mathrm{CS}_{1}}^{1}(\mathbf{X})$ and $\nu_{2}+N\left(F_{2}\right) \mu_{H} \in S_{1}(\mathbf{X})$. Let $A=A^{\nu}+A^{F}$. If $\mathbf{E}_{x}^{w}\left[e_{A}\left(\zeta^{w}\right)\right]$ is finite for some $(x, w) \in(E \times E) \backslash \mathrm{d}$, then it is bounded on $(E \times E) \backslash \mathrm{d}$.

Proof. The proof is similar to that of [4, Theorem 3.8], [10, Theorem 3.5]. We provide the proof for reader's convenience. We let $u(x, y):=$ $\mathbf{E}_{x}^{y}\left[e_{A}\left(\zeta^{y}\right)\right]$. By symmetricity (see $\left.[10,(3.7)]\right), u(x, y)=u(y, x)$ for $(x, y) \in$ $(E \times E) \backslash \mathrm{d}$. Set $O:=\{(x, z) \in(E \times E) \backslash \mathrm{d} \mid u(x, z)<\infty\}$ and suppose $O \neq \emptyset$. Take $\left(x_{0}, y_{0}\right) \in O$. The condition $\left(\nu_{1}, e^{F_{1}}-1\right) \in B_{\mathrm{CS}_{1}}^{1}(\mathbf{X})$ yields $\nu_{1}+$ $N^{z}\left(e^{F_{1}}-1\right) \mu_{H} \in S_{\mathrm{CK}_{1}}^{1}\left(\mathbf{X}^{z}\right) \subset S_{\mathrm{NK}_{1}}^{1}\left(\mathbf{X}^{z}\right)$ for each $z \in E$. Here $N^{z}(y, \mathrm{~d} w):=$ $\frac{R(w, z)}{R(y, z)} N(y, \mathrm{~d} w)$ is the Lévy kernel of $\mathbf{X}^{z}$ (see [10, Proposition 3.3]). We will confirm it. Since $\left(\nu_{1}, e^{F_{1}}-1\right) \in B_{\mathrm{CS}_{1}}^{1}(\mathbf{X}) \subset B_{\mathrm{DS}_{0}}^{1}(\mathbf{X})=S_{\mathrm{DS}_{0}}^{1}(\mathbf{X}) \times A_{\mathrm{DS}_{0}}^{1}(\mathbf{X})$, we see $\nu_{1}+N^{z}\left(e^{F_{1}}-1\right) \mu_{H} \in S_{D_{0}}^{1}\left(\mathbf{X}^{z}\right)$ for any $z \in E$. Applying [4, Proposition 2.4(2)] to $\mathbf{X}^{z}$, it suffices to show that for each $z \in E$ and any decreasing sequence $\left\{D_{n}\right\}$ of Borel subsets of $E^{z}$ with empty intersection,

$$
\lim _{n \rightarrow \infty} \sup _{x \in E^{z}} \int_{D_{n}} R^{z}(x, y)\left(\nu_{1}+N^{z}\left(e^{F_{1}}-1\right) \mu_{H}\right)(\mathrm{d} y)<1 .
$$

By Lemma 6.2(2), we have

$$
\begin{aligned}
& \lim _{n \rightarrow \infty} \sup _{(x, z) \in(E \times E) \backslash \mathrm{d}}\left(\int_{D_{n}} \frac{R(x, y) R(y, z)}{R(x, z)} \nu_{1}(\mathrm{~d} y)\right. \\
& \left.\quad+\int_{\left(D_{n} \times E\right) \cup\left(E \times D_{n}\right)} \frac{R(x, y) R(w, z)}{R(x, z)}\left(e^{F_{1}(y, w)}-1\right) N(y, \mathrm{~d} w) \mu_{H}(\mathrm{~d} y)\right)<1 .
\end{aligned}
$$

Hence,

$$
\begin{aligned}
\lim _{n \rightarrow \infty} & \sup _{x \in E^{z}} \int_{D_{n}} R^{z}(x, y)\left(\nu_{1}+N^{z}\left(e^{F_{1}}-1\right) \mu_{H}\right)(\mathrm{d} y) \\
= & \lim _{n \rightarrow \infty} \sup _{x \in E^{z}}\left(\int_{D_{n}} \frac{R(x, y) R(y, z)}{R(x, z)} \nu_{1}(\mathrm{~d} y)\right. \\
& \left.+\int_{D_{n}} \int_{E} \frac{R(x, y) R(y, z)}{R(x, z)}\left(e^{F_{1}(y, w)}-1\right) N^{z}(y, \mathrm{~d} w) \mu_{H}(\mathrm{~d} y)\right)
\end{aligned}
$$




$$
\begin{aligned}
\leq & \lim _{n \rightarrow \infty} \sup _{(x, z) \in(E \times E) \backslash \mathrm{d}}\left(\int_{D_{n}} \frac{R(x, y) R(y, z)}{R(x, z)} \nu_{1}(\mathrm{~d} y)\right. \\
& \left.+\int_{\left\{D_{n} \times E\right\} \cup\left\{E \times D_{n}\right\}} \frac{R(x, y) R(w, z)}{R(x, z)}\left(e^{F_{1}(y, w)}-1\right) N(y, \mathrm{~d} w) \mu_{H}(\mathrm{~d} y)\right)<1 .
\end{aligned}
$$

Thus, we have (6.3). One can apply Theorem 5.1 to $\mathbf{X}^{y_{0}}$ so that

$$
\sup _{x \in E^{y_{0}}} u\left(x, y_{0}\right)=\sup _{x \in E^{y_{0}}} u\left(y_{0}, x\right)<\infty .
$$

Applying Theorem 5.1 to $\mathbf{X}^{x}$ again that for any $x \in E$,

$$
\sup _{z \in E^{x}} u(x, z)=\sup _{z \in E^{x}} u(z, x)<\infty .
$$

Recall the condition $\left(\nu_{1}, e^{F_{1}}-1\right) \in B_{\mathrm{CS}_{1}}^{1}(\mathbf{X})$. There exists a Borel subset $K$ of finite $\left(\nu_{1}+N\left(e^{F_{1}}-1\right) \mu_{H}\right)$-measure such that there exists $\delta>0$ with

$$
\begin{aligned}
\beta:= & \sup _{\substack{B \subset K,\left(\nu_{1}+N\left(e^{\left.F_{1}-1\right) \mu_{H}}\right)(B)<\delta\right.}} \sup _{(x, z) \in(E \times E) \backslash \mathrm{d}}\left(R^{z} \mathbf{1}_{B \cup K^{c} \nu_{1}(x)}\right. \\
& \left.+\int_{\{(K \backslash B) \times(K \backslash B)\}^{c}} \frac{R(x, y)\left|e^{F_{1}(y, w)}-1\right| R(w, z)}{R(x, z)} N(y, \mathrm{~d} w) \mu_{H}(\mathrm{~d} y)\right)<1 .
\end{aligned}
$$

Note that $u(x, y)$ is $\mathcal{B}(E \times E)$-measurable if we set $u(x, y)=1$ for $(x, y) \in \mathrm{d}$. Hence, $\left\{x \in K \mid \sup _{z \in E} u(x, z) \geq n\right\}$ is $\mathcal{B}(E)$-measurable as it is the $x$-projection of the set $\{(x, z) \in K \times E \mid u(x, z) \geq n\}$. As $\bigcap_{n=2}^{\infty}\{x \in K \mid n \leq$ $\left.\sup _{z \in E} u(x, z)\right\}=\emptyset$ from (6.4), we can choose $N$ large enough so that the set $B_{N}:=\left\{x \in K \mid N \leq \sup _{z \in E} u(x, z)\right\}$ has $\left(\nu_{1}+N\left(e^{F_{1}}-1\right) \mu_{H}\right)$-measure less than $\delta$. Applying Khasinskii's Lemma to $\mathbf{X}^{z}$, we have

$$
\begin{aligned}
& C:=\sup _{(x, z) \in(E \times E) \backslash \mathbf{d}} \mathbf{E}_{x}^{z}\left[\exp \left(A_{\tau_{B_{N} \cup K^{c}}}^{\nu_{1}}+A_{\tau_{B_{N} \cup K^{c}}}^{F_{1}}\right)\right] \\
& =\sup _{(x, z) \in(E \times E) \backslash \mathrm{d}} \mathbf{E}_{x}^{z}\left[\operatorname{Exp}\left(A^{\nu_{1}}+A^{e^{F_{1}}-1}\right)_{\tau_{B_{N} \cup K^{c}}}\right] \\
& \leq \frac{1}{1-\sup _{(x, z) \in(E \times E) \backslash \mathrm{d}} \mathbf{E}_{x}^{z}\left[A_{\tau_{B_{N}} \cup K^{c}}^{\nu_{1}}+A_{\tau_{B_{N} \cup K^{c}}}^{F_{1}-1}\right.} \leq \frac{1}{1-\beta}<\infty .
\end{aligned}
$$

By the formula

$$
\begin{aligned}
u(x, z)= & \mathbf{E}_{x}^{z}\left[\exp \left(A_{\tau_{B_{N} \cup K^{c}}}^{\nu}+A_{\tau_{B_{n} \cup K^{c}}}^{F}\right): \tau_{B_{N} \cup K^{c}}=\zeta^{z}\right] \\
& +\mathbf{E}_{x}^{z}\left[\exp \left(A_{\tau_{B_{N} \cup K^{c}}}^{\nu}+A_{\tau_{B_{N} \cup K^{c}}}^{F}\right) u\left(X_{\tau_{B_{N} \cup K^{c}}}, z\right): \tau_{B_{N} \cup K^{c}}<\zeta^{z}\right]
\end{aligned}
$$

for $(x, z) \in(E \times E) \backslash \mathrm{d}$, we have $u(x, z) \leq C+C N$ for $(x, z) \in O \cap\left(B_{N} \times E\right)$ in view of $X_{\tau_{B_{N} \cup K^{c}}} \in O^{z} \cap\left(K \backslash B_{N}\right) \mathbf{P}_{x}^{z}$-a.s. on $\left\{\tau_{B_{N} \cup K^{c}}<\zeta^{z}\right\}$ for $x \in O^{z}$, where we use the absorbing property of $O^{z}:=\{x \in E \mid u(x, z)<\infty\}$ under $\mathbf{X}^{z}$ for each $z \in E$. Moreover, $u(x, z) \leq N$ for $(x, z) \in O \cap\left(B_{N}^{c} \times E\right)$. Thus, we obtain $O=\{(x, z) \in(E \times E) \backslash \mathrm{d} \mid u(x, z) \leq C+C N\}$ for some $C>0$ and $N \in \mathbb{N}$ under $O \neq \emptyset$, which implies the assertion. 
LEMmA 6.5 (cf. [4, Lemmas 3.5 and 3.9]). Suppose that $\nu_{1}+N\left(F_{1}\right) \mu_{H} \in$ $S_{1}(\mathbf{X}), \nu_{2}+N\left(F_{2}\right) \mu_{H} \in S_{1}(\mathbf{X})$ and $\mu_{\langle u\rangle} \in S_{D}^{1}(\mathbf{X})$. Let $A=A^{\nu}+A^{F}+N^{u}$. Then the following hold:

(1) For every Borel function $f \geq 0$,

$$
\mathbf{E}_{x}\left[\int_{0}^{\infty} e_{A}(t) f\left(X_{t}\right) \mathrm{d} t\right]=\int_{E} R(x, y) \mathbf{E}_{x}^{y}\left[e_{A}\left(\zeta^{y}\right)\right] f(y) \mathfrak{m}(\mathrm{d} y) .
$$

(2) For any positive measure $\mu \in S_{D_{0}}^{1}(\mathbf{X})$,

$$
\mathbf{E}_{x}\left[\int_{0}^{\infty} e_{A}(t) \mathrm{d} A_{t}^{\mu}\right]=\int_{E} R(x, y) \mathbf{E}_{x}^{y}\left[e_{A}\left(\zeta^{y}\right)\right] \mu(\mathrm{d} y) .
$$

Here $A^{\mu}$ is the PCAF of $\mathbf{X}$ in the strict sense with Revuz measure $\mu$.

Proof. The proof is similar to that of [4, Lemmas 3.5 and 3.9]. We provide it for completeness. It suffices to prove (1). The proof for (2) is similar. Fix a bounded Borel measurable function $f_{0} \in L^{2}(E ; \mathfrak{m})$ being strictly positive $\mathfrak{m}$ a.e. on $E$ such that $h:=R f_{0}$ is bounded on $E$. The existence of such $f_{0}$ is proved in Getoor [21]. As in the proof of [4, Lemma 3.5],

$$
M_{t}:=h\left(X_{t}\right)-h\left(X_{0}\right)+\int_{0}^{t} f_{0}\left(X_{s}\right) \mathrm{d} s
$$

is a square integrable martingale under $\mathbf{P}_{x}$ with

$$
\sup _{t \in[0, \infty[} \mathbf{E}_{x}\left[M_{t}^{2}\right]<\infty
$$

for all $x \in E$. By [20, Proposition 5.3], for any bounded $\mathcal{F}_{\infty}$-measurable $\Lambda$

$$
\int_{E} R(x, y) \mathbf{E}_{x}^{y}[\Lambda] f_{0}(y) \mathfrak{m}(\mathrm{d} y)=h(x) \mathbf{E}_{x}^{h}[\Lambda] .
$$

Applying the bounded $\mathcal{F}_{\infty}$-measurable $\Lambda=e_{A}(\infty) \wedge k$ to $(6.6)$,

$$
\int_{E} R(x, y) \mathbf{E}_{x}^{y}\left[e_{A}\left(\zeta^{y}\right) \wedge k\right] f_{0}(y) \mathfrak{m}(\mathrm{d} y)=h(x) \mathbf{E}_{x}^{h}\left[e_{A}\left(\zeta^{h}\right) \wedge k\right] .
$$

By way of the argument in Sharpe [33, Section 62], we have

$$
\int_{E} R(x, y) \mathbf{E}_{x}^{y}\left[e_{A}\left(\zeta^{y}\right) \wedge k\right] f_{0}(y) \mathfrak{m}(\mathrm{d} y)=\mathbf{E}_{x}\left[\int_{0}^{\infty}\left(e_{A}(t) \wedge k\right) f_{0}\left(X_{t}\right) \mathrm{d} t\right]
$$

as in the proof of [4, Lemma 3.5]. Letting $k \rightarrow \infty$, we obtain the assertion for $f=f_{0}$. The rest of the proof is similar to that of [4, Lemma 3.5].

TheOREM 6.2 (cf. [4, Theorem 3.10]). Suppose that $\left(\nu_{1}, e^{F_{1}}-1\right) \in B_{\mathrm{CS}_{1}}^{1}(\mathbf{X})$, $\nu_{2}+N\left(F_{2}\right) \mu_{H} \in S^{1}(\mathbf{X})$. Let $A=A^{\nu}+A^{F}$. Then the following are equivalent:

(1) $(\mathbf{X}, A)$ is gaugeable.

(2) $(\mathbf{X}, A)$ is conditionally gaugeable. 
Proof. The proof is similar to that of [4, Theorem 3.10] with [4, Lemma 3.9] by use of Lemmas 4.2(2), 6.5(3), (4) and Theorem 6.1. We omit it.

We also obtain the existence of $\alpha$-order resolvent kernel $R_{\alpha}^{U, \bar{\nu}+F}(x, y)$ of the Feynman-Kac semigroup $P_{t}^{U, \bar{\nu}+F}$ defined by

$$
P_{t}^{U, \bar{\nu}+F} f(x):=\mathbf{E}_{x}^{U}\left[\exp \left(A_{t}^{\bar{\nu}}+A_{t}^{F}\right) f\left(X_{t}\right)\right] .
$$

$R_{\alpha}^{U, \bar{\nu}+F}(x, y)$ is defined for all $x, y \in E$ (but possibly infinite) and for each $x \in E, y \mapsto R_{\alpha}^{U, \bar{\nu}+F}(x, y)$ is finely continuous with respect to $\mathbf{U}$ by applying Lemma 6.3 to $\mathbf{U}$, because $e^{-2 u}\left(\nu_{1}+N\left(e^{U}\left(e^{F_{1}}-1\right)\right) \mu_{H}\right) \in S_{\mathrm{EK}}^{1}(\mathbf{U})$ and $e^{-2 u}\left(\mu_{2}+N\left(e^{U} F_{2}\right) \mu_{H}\right) \in S_{1}(\mathbf{U})$ hold by Lemma 4.1. By (4.10), we have the relation

$$
R^{A}(x, y)=R^{U, \bar{\nu}+F}(x, y) e^{-u(x)-u(y)}
$$

holds for m-a.e. $y \in E$ and all $x \in E$. Applying Lemma 3.1 to $\mathbf{U},(6.7)$ holds for all $x, y \in E$.

Proof of Theorem 1.2. The equivalence $(1) \Longleftrightarrow(5)$ also follows from Theorem 1.1 and

$$
\lambda^{\mathcal{Q}}\left(\bar{\mu}_{1}\right)=\inf \left\{\mathcal{Q}(R(\cdot, y) f, R(\cdot, y) f) \mid R(\cdot, y) f \in \mathcal{C}, \int_{E} f^{2} R(\cdot, y)^{2} \mathrm{~d} \bar{\mu}_{1}=1\right\} .
$$

It suffices to prove the equivalence $(1) \Longleftrightarrow(2) \Longleftrightarrow(3) \Longleftrightarrow(4)$. By Lemma 6.5, we see that

$$
R^{A}(x, y)=R(x, y) \mathbf{E}_{x}^{y}\left[e_{A}\left(\zeta^{y}\right)\right] \quad \text { for } \mathfrak{m} \text {-a.e. } y \in E^{x} .
$$

The implications $(4) \Longrightarrow(3) \Longrightarrow(2)$ are trivial. Suppose (2). Owing to the symmetricity $R^{A}(x, y)=R^{A}(y, x)$, this is equivalent to that for each $y \in E, R^{A}(x, y)<\infty$ for $\mathfrak{m}$-a.e. $x \in E^{y}$. Then $\mathbf{E}_{x}^{y}\left[e_{A}\left(\zeta^{y}\right)\right]=\frac{R^{A}(x, y)}{R(x, y)}<\infty$ for m-a.e. $x \in E^{y}$ for each $y \in E$. Here we use $R(x, y)>0$ for $x, y \in E$ in view of Lemma 6.1. Applying Theorem 5.1 to $\mathbf{X}^{y}$, we have from $\bar{\mu}_{1}^{*} \in S_{\mathrm{CS}_{1}}^{1}(\mathbf{X}) \subset \operatorname{semi}-S_{\mathrm{CS}_{1}}^{1}(\mathbf{X}), \mu_{\langle u\rangle} \in S_{\mathrm{CS}_{\infty}}^{1}(\mathbf{X}) \subset \operatorname{semi}-S_{\mathrm{CS}_{\infty}}^{1}(\mathbf{X})$ and $\mu_{2}+$ $N\left(F_{2}\right) \mu_{H} \in S_{\mathrm{DS}_{0}}^{1}(\mathbf{X}) \subset \operatorname{semi}-S_{\mathrm{DS}_{0}}^{1}(\mathbf{X})$ that

$$
\sup _{x \in E^{y}} \mathbf{E}_{x}^{y}\left[e_{A}\left(\zeta^{y}\right)\right]<\infty .
$$

Consequently (1) holds from (6.8). Conversely suppose (1). Note that

$$
\inf _{x \in E^{y}} \mathbf{E}_{x}^{y}\left[e_{A}\left(\zeta^{y}\right)\right]>0
$$

always holds under the present conditions in view of Corollary 5.3. Then there exists $C>0$ depending only on $y \in E$ such that $C^{-1} R(x, y) \leq R^{A}(x, y) \leq$ $C R(x, y)$ m-a.e. $x \in E^{y}$. Noting the fine continuity of $x \mapsto R^{A}(x, y)$ by Lemma 6.3, we obtain (4). The equivalence $(6) \Longleftrightarrow(5)$ under $\bar{\mu}_{1}^{*} \in S_{\mathrm{CS}_{1}}^{1}(\mathbf{X})$, $\mu_{\langle u\rangle} \in S_{\mathrm{CS}_{\infty}}^{1}(\mathbf{X})$ and $\mu_{2}+N\left(F_{2}\right) \mu_{H} \in S_{\mathrm{DS}_{0}}^{1}(\mathbf{X})$ follows from Theorem 1.1, because $S_{\mathrm{CS}_{1}}^{1}(\mathbf{X}) \subset S_{\mathrm{CK}_{1}}^{1}(\mathbf{X})$ and $S_{\mathrm{DS}_{0}}^{1}(\mathbf{X}) \subset S_{D_{0}}^{1}(\mathbf{X})$. Next, we prove $(6) \Longrightarrow$ 
$(8) \Longrightarrow(7) \Longrightarrow(1)$ under (1.4) with $\left(\mu_{1}, F_{1}\right) \in B_{\mathrm{CS}_{\infty}}^{1}(\mathbf{X}),\left(\mu_{2}, F_{2}\right) \in B_{\mathrm{DS}_{0}}^{1}(\mathbf{X})$ and $\mu_{\langle u\rangle} \in S_{\mathrm{CS}_{\infty}}^{1}(\mathbf{X})$. The proof for $(6) \Longrightarrow(8) \Longrightarrow(7) \Longrightarrow(1)$ under $u=0$, $\left(\mu_{1}, e^{F_{1}}-1\right) \in B_{\mathrm{CS}_{1}}^{1}(\mathbf{X})$ and $\left(\mu_{2}, F_{2}\right) \in B_{\mathrm{DS}_{0}}^{1}(\mathbf{X})$ is simpler than it. The proof for $(6) \Longrightarrow(7) \Longrightarrow(1)$ under $u=0$ with $\left(\mu_{1}, e^{F_{1}}-1\right) \in B_{\mathrm{CS}_{1}}^{1}(\mathbf{X})$ without $\left(\mu_{2}, F_{2}\right) \in B_{\mathrm{DS}_{0}}^{1}(\mathbf{X})$ is also similar. Suppose (6). As we proved in Theorem 1.1 with (4.11), the condition (5) is equivalent to the gaugeability $\sup _{x \in E} \mathbf{E}_{x}^{U}\left[\exp \left(A_{\zeta}^{\bar{\nu}}+A_{\zeta}^{F}\right)\right]<\infty$. Recall $\bar{\nu}_{1}:=\mu_{1}+N\left(e^{U}-U-1\right) \mu_{H}+$ $\frac{1}{2} \mu_{\langle u\rangle}^{c}$ and $\bar{\nu}_{2}:=\mu_{2}$. Note that $\left(e^{-2 u} \bar{\nu}_{1}, e^{-u(x)-u(y)} F_{1}(x, y)\right) \in B_{\mathrm{CS}_{\infty}}^{1}(\mathbf{U})$ and $\left(e^{-2 u} \mu_{2}, e^{-u(x)-u(y)} F_{2}(x, y)\right) \in B_{\mathrm{DS}_{0}}^{1}(\mathbf{U})$ hold under (1.4). From these facts with Theorem 6.2, we see that $\sup _{x \in E} \mathbf{E}_{x}^{U}\left[\exp \left(A_{\zeta}^{\bar{\nu}}+A_{\zeta}^{F}\right)\right]<\infty$ is equivalent to the following conditional gaugeability:

$$
\sup _{(x, y) \in(E \times E) \backslash \mathrm{d}}\left(\mathbf{E}_{x}^{U}\right)^{y}\left[\exp \left(A_{\zeta^{y}}^{\bar{\nu}}+A_{\zeta^{y}}^{F}\right)\right]<\infty,
$$

where $\left(\mathbf{E}_{x}^{U}\right)^{y}$ stands for the expectation of Doob's $R^{U}(\cdot, y)$-transformed process $\mathbf{U}^{y}$ of $\mathbf{U}$ starting from $x \in E^{y}$. Since $\left(e^{-2 u} \mu_{2}, e^{-u(x)-u(y)} F_{2}(x, y)\right) \in$ $B_{\mathrm{DS}_{0}}^{1}(\mathbf{U})$ under (1.4)

$$
\begin{aligned}
& \inf _{(x, y) \in(E \times E) \backslash \mathrm{d}}\left(\mathbf{E}_{x}^{U}\right)^{y}\left[\exp \left(A_{\zeta^{y}}^{\bar{\nu}}+A_{\zeta^{y}}^{F}\right)\right] \\
& \geq \exp \left(-\sup _{(x, y) \in(E \times E) \backslash \mathrm{d}}\left(\mathbf{E}_{x}^{U}\right)^{y}\left[A_{\zeta^{y}}^{\mu_{2}}+A_{\zeta^{y}}^{F_{2}}\right]\right)>0 .
\end{aligned}
$$

Moreover, by virtue of Lemma 6.5(1)

$$
R^{U, \bar{\nu}+F}(x, y)=R^{U}(x, y)\left(\mathbf{E}_{x}^{U}\right)^{y}\left[\exp \left(A_{\zeta^{y}}^{\bar{\nu}}+A_{\zeta^{y}}^{F}\right)\right] \quad \text { for m-a.e. } y \in E^{x} .
$$

Therefore, there exists $C>0$ such that

$$
C^{-1} R^{U}(x, y) \leq R^{U, \bar{\nu}+F}(x, y) \leq C R^{U}(x, y) \quad \text { m-a.e. } y \in E^{x} .
$$

Applying Lemma 6.3 to $\mathbf{U}$, (6.10) holds for all $y \in E^{x}$, because the underlying symmetric measure $e^{-2 u} \mathfrak{m}$ has always full fine support under (AC). Now, by using the relation (6.7) with the boundedness of $u$, we obtain (8). Next suppose (8). Then by (6.8), we have that there exists $C>0$ independent of $x, y \in E$ such that $C^{-1} \leq \mathbf{E}_{x}^{y}\left[e_{A}\left(\zeta^{y}\right)\right] \leq C$ m-a.e. $x \in E^{y}$. Applying Lemma 6.4, $\mathbf{E}_{x}^{y}\left[e_{A}\left(\zeta^{y}\right)\right] \leq C$ holds for all $x \in E^{y}$ and $y \in E$, which implies (7). The implication $(7) \Longrightarrow(1)$ is trivial.

Finally, we give a criterion for (1.4). The next theorem is a slight extension of [4, Theorem 3.8].

Theorem 6.3 (Conditional Gauge Theorem II, [26, Theorem 6.3]). Suppose $\mu_{\langle u\rangle}^{c}=\mu_{\langle u\rangle}^{\kappa}=0, \mu=\mu_{1}-\mu_{2} \in S_{\mathrm{CS}_{1}}^{1}(\mathbf{X})-S_{\mathrm{DS}_{0}}^{1}(\mathbf{X})$ and $F=F_{1}-F_{2}$ with $F_{1}+U_{+} \in A_{\mathrm{CS}_{\infty}}^{1}(\mathbf{X})$ and $F_{2}+U_{-} \in A_{\mathrm{DS}_{0}}^{1}(\mathbf{X})$. Here $U_{ \pm}(x, y):=(u(x)-$ $u(y))^{ \pm}, x, y \in E$. Then the following are equivalent to each other.

(1) There exists $(x, y) \in(E \times E) \backslash \mathrm{d}$ such that $\mathbf{E}_{x}^{y}\left[e_{A}\left(\zeta^{y}\right)\right]<\infty$. 
(2) $\sup _{(x, y) \in(E \times E) \backslash \mathrm{d}} \mathbf{E}_{x}^{y}\left[e_{A}\left(\zeta^{y}\right)\right]<\infty$.

Proposition 6.1. Suppose $\mu_{\langle u\rangle}^{c}=\mu_{\langle u\rangle}^{\kappa}=0, U_{+} \in A_{\mathrm{CS}_{\infty}}^{1}(\mathbf{X}) \cap J_{\mathrm{DS}_{0}}^{1}(\mathbf{X})$ and $U_{-} \in A_{\mathrm{DS}_{0}}^{1}(\mathbf{X}) \cap J_{\mathrm{CS}_{1}}^{1}(\mathbf{X})$. Then there exists $C>0$ such that (1.4) holds.

Proof. The proof is similar to the proof of [26, Proposition 6.1] by replacing $Y_{1}^{1}$ with $U_{t}$. So we omit it.

ExAmPle 6.1 (Symmetric Relativistic $\alpha$-stable Process). Take $0<\alpha<2$ and $m \geq 0$. Let $\mathbf{X}=\left(\Omega, X_{t}, \mathbf{P}_{x}\right)$ be a Lévy process on $\mathbb{R}^{d}$ with

$$
\mathbf{E}_{0}\left[e^{\sqrt{-1}\left\langle\xi, X_{t}\right\rangle}\right]=\exp \left(-t\left\{\left(|\xi|^{2}+m^{2 / \alpha}\right)^{\alpha / 2}-m\right\}\right) .
$$

If $m>0$, it is called the relativistic $\alpha$-stable process with mass $m$ (see [31]). In particular, if $\alpha=1$ and $m>0$, it is called the relativistic free Hamiltonian process (see [23]). When $m=0, \mathbf{X}$ is nothing but the usual (rotationally) symmetric $\alpha$-stable process. It is known that $\mathbf{X}$ is transient if and only if $d>2$ under $m>0$ or $d>\alpha$ under $m=0$, and $\mathbf{X}$ is a doubly Feller conservative process.

Let $(\mathcal{E}, \mathcal{F})$ be the Dirichlet form on $L^{2}\left(\mathbb{R}^{d}\right)$ associated with $\mathbf{X}$. Using Fourier transform $\hat{f}(x):=\frac{1}{(2 \pi)^{d / 2}} \int_{\mathbb{R}^{d}} e^{i\langle x, y\rangle} f(y) \mathrm{d} y$, it follows from Example 1.4 .1 of $[19]$ that

$$
\left\{\begin{array}{l}
\mathcal{F}:=\left\{\left.f \in L^{2}\left(\mathbb{R}^{d}\right)\left|\int_{\mathbb{R}^{d}}\right| \hat{f}(\xi)\right|^{2}\left(\left(|\xi|^{2}+m^{2 / \alpha}\right)^{\alpha / 2}-m\right) \mathrm{d} \xi<\infty\right\} \\
\mathcal{E}(f, g):=\int_{\mathbb{R}^{d}} \hat{f}(\xi) \overline{\hat{g}}(\xi)\left(\left(|\xi|^{2}+m^{2 / \alpha}\right)^{\alpha / 2}-m\right) \mathrm{d} \xi \quad \text { for } f, g \in \mathcal{F}
\end{array}\right.
$$

It is shown in [11] that the corresponding jumping measure satisfies

$$
J(\mathrm{~d} x \mathrm{~d} y)=J_{m}(x, y) \mathrm{d} x \mathrm{~d} y \quad \text { with } J_{m}(x, y)=A(d,-\alpha) \frac{\Psi\left(m^{1 / \alpha}|x-y|\right)}{|x-y|^{d+\alpha}},
$$

where $A(d,-\alpha)=\frac{\alpha 2^{d+\alpha} \Gamma\left(\frac{d+\alpha}{2}\right)}{2^{d+1} \pi^{d / 2} \Gamma\left(1-\frac{\alpha}{2}\right)}$, and $\Psi(r):=I(r) / I(0)$, where

$$
I(r):=\int_{0}^{\infty} s^{\frac{d+\alpha}{2}-1} e^{-\frac{s}{4}-\frac{r^{2}}{s}} \mathrm{~d} s
$$

is a function satisfying $\Psi(r) \asymp e^{-r}\left(1+r^{(d+\alpha-1) / 2}\right)$ near $r=\infty$, and $\Psi(r)=$ $1+\Psi^{\prime \prime}(0) r^{2} / 2+o\left(r^{4}\right)$ near $r=0$. In particular,

$$
\left\{\begin{array}{l}
\mathcal{F}=\left\{f \in L^{2}\left(\mathbb{R}^{d}\right)\left|\int_{\mathbb{R}^{d} \times \mathbb{R}^{d}}\right| f(x)-\left.f(y)\right|^{2} J_{m}(x, y) \mathrm{d} x \mathrm{~d} y<\infty\right\}, \\
\mathcal{E}(f, g):=\frac{1}{2} \int_{\mathbb{R}^{d} \times \mathbb{R}^{d}}(f(x)-f(y))(g(x)-g(y)) J_{m}(x, y) \mathrm{d} x \mathrm{~d} y \quad \text { for } f, g \in \mathcal{F} .
\end{array}\right.
$$

Let $p_{t}(x, y)$ be the heat kernel of $\mathbf{X}$. The following global heat kernel estimates were proved in [15, Theorem 2.1]: There exists $C_{1}, C_{2}>0$ such that

$$
C_{2}^{-1} \Phi_{1 / C_{1}}^{m}(t, x, y) \leq p_{t}(x, y) \leq C_{2} \Phi_{C_{1}}^{m}(t, x, y)
$$


where

$$
\Phi_{C}^{m}(t, x, y)= \begin{cases}t^{-d / \alpha} \wedge t J_{m}(x, y), & t \in] 0,1 / m], \\ m^{d / \alpha-d / 2} t^{-d / 2} e^{\left(-C^{-1}\left(m^{1 / \alpha}|x-y| \wedge m^{2 / \alpha-1} \frac{|x-y|^{2}}{t}\right)\right)}, & t \in] 1 / m, \infty[.\end{cases}
$$

For a signed Borel measure $\mu$ on $\mathbb{R}^{d}, \mu$ is said to be of Kato class with respect to $\mathbf{X}$ if and only if

$$
\begin{aligned}
& \lim _{r \rightarrow 0} \sup _{x \in \mathbb{R}^{d}} \int_{|x-y|<r} \frac{|\mu|(\mathrm{d} y)}{|x-y|^{d-\alpha}}=0 \text { for } d>\alpha, \\
& \lim _{r \rightarrow 0} \sup _{x \in \mathbb{R}^{d}} \int_{|x-y|<r}\left(\log |x-y|^{-1}\right)|\mu|(\mathrm{d} y)=0 \quad \text { for } d=\alpha(=1), \\
& \sup _{x \in \mathbb{R}^{d}} \int_{|x-y| \leq 1}|\mu|(\mathrm{d} y)<\infty \quad \text { for } \alpha>d(=1) .
\end{aligned}
$$

Denote by $K_{d, \alpha}$ the family of nonnegative measures of Kato class with respect to $\mathbf{X}$. Then we have $K_{d, \alpha}=S_{K}^{1}(\mathbf{X})$ by [29].

From now on, we assume the transience of $\mathbf{X}$. Using (6.11), we see the following Green kernel estimate by [15, Theorem 1.3]: For each fixed $M>0$, there exists $C=C(d, \alpha, M)>1$ such that for any $m \in] 0, M], x, y \in \mathbb{R}^{d}$

$$
C^{-1} \frac{1+m^{\frac{2-\alpha}{\alpha}}|x-y|^{2-\alpha}}{|x-y|^{d-\alpha}} \leq R(x, y) \leq C \frac{1+m^{\frac{2-\alpha}{\alpha}}|x-y|^{2-\alpha}}{|x-y|^{d-\alpha}} .
$$

From this, we can obtain that $3 G$-inequality holds. Owing to this $3 G$-inequality, we have $S_{\mathrm{CK}_{\infty}}^{1}(\mathbf{X})=S_{\mathrm{CS}}^{1}(\mathbf{X})$ and $S_{D_{0}}^{1}(\mathbf{X})=S_{\mathrm{DS}_{0}}^{1}(\mathbf{X})$.

From [31, Lemma 3] or $(6.11)$, for $m>0$, there exists $C_{1}(d, m, \alpha)>0$ depending only on $m, d$ and $\alpha$ such that

$$
\sup _{x, y \in \mathbb{R}^{d}} p_{t}(x, y) \leq C_{1}(d, m, \alpha) t^{-d / 2} \quad \text { for any } t \geq 1 .
$$

We can apply $[17$, Lemma $5.1(2)]$ to $\mathbf{X}$ for $\Phi_{1}:=C_{1} \Psi_{1}, \Phi_{2}(s):=\frac{C_{2} e^{m}}{(1+s)^{d+\alpha}}$ and $\Phi_{2}^{*}(s):=C(d, m, \alpha)$ with $t_{0}=1, d^{*}=d$ and $\beta^{*}=2>\beta=\alpha$ provided $m>0$, and for $\Phi_{1}=C_{1} \Psi_{2}, \Phi_{2}=\Phi_{2}^{*}=C_{2} \Psi_{2}$ with $\Psi_{2}(s):=1 /(1+s)^{d+\alpha}, d=d^{*}$ and $\beta^{*}=\beta=\alpha$ provided $m=0$. Hence every $\mu \in K_{d, \alpha}=S_{K}^{1}(\mathbf{X})$ with $\mu\left(\mathbb{R}^{d}\right)<\infty$ belongs to $S_{K_{\infty}^{+}}^{1}(\mathbf{X})$ (to $S_{K_{\infty}}^{1}(\mathbf{X})$ if $\mathbf{X}$ is transient).

We show that there exists a positive Radon measure $\nu \in S_{D_{0}}^{1}(\mathbf{X}) \backslash S_{K}^{1}(\mathbf{X})$ under the transience of $\mathbf{X}$. More concretely, in [8, Example 4.2] for $m=0$ and in [26, Example 4.2] for $m>0$, we construct a nonnegative function $\varphi \in L^{1}\left(\mathbb{R}^{d}\right)$ such that $\varphi(x) \mathrm{d} x \notin S_{K}^{1}(\mathbf{X})$ and $\left\|R^{(\alpha)} \varphi\right\|_{\infty}<\infty$. Here $R^{(\alpha)} \varphi(x):=$ $\int_{\mathbb{R}^{d}} \frac{\varphi(y)}{|x-y|^{d-\alpha}} \mathrm{d} y$ is the Riesz potential of $\varphi$.

Take $u \in \mathcal{F}_{e} \cap C_{\infty}\left(\mathbb{R}^{d}\right)$ with $\left(\int_{\mathbb{R}^{d}}(u(x)-u(y))^{2} J_{m}(x, y) \mathrm{d} y\right) \mathrm{d} x \in K_{d, \alpha}$. Since this energy measure has finite total mass, it also belongs to $S_{K_{\infty}}^{1}(\mathbf{X})$. We know the existence of the limit $N_{\infty}^{u}:=\lim _{t \rightarrow \infty} N_{t}^{u} \mathbf{P}_{x}$-a.s. under the transience and the doubly Feller property of $\mathbf{X}$. Indeed, there exists a strictly 
positive bounded Borel function $g$ on $\mathbb{R}^{d}$ such $R g$ is bounded by [21]. Moreover, $R g \in C_{b}\left(\mathbb{R}^{d}\right)$ in view of the doubly Feller property of $\mathbf{X}$. Let $\left\{K_{n}\right\}$ be an increasing sequence of compact subsets of $\mathbb{R}^{d}$ with $\mathbb{R}^{d}=\bigcup_{k=1}^{\infty} K_{n}$. Then $c_{n}:=\inf _{x \in K_{n}} R g(x)>0$ and $p_{K_{n}}(x):=\mathbf{P}_{x}\left(\sigma_{K_{n}}<\infty\right) \leq \mathbf{E}_{x}\left[R g\left(X_{\sigma_{K_{n}}}\right)\right] / c_{n} \leq$ $R g(x) / c_{n}<\infty$. Then $P_{t} p_{K_{n}}(x) \leq P_{t} R g(x) / c_{n} \rightarrow 0$ as $t \rightarrow \infty$, and consequently,

$$
\mathbf{P}_{x}\left(\bigcap_{j=1}^{\infty} \Lambda_{j}\right)=\lim _{j \rightarrow \infty} \mathbf{P}_{x}\left(\Lambda_{j}\right)=0 \text { for } \Lambda_{j}:=\left\{\sigma_{K_{n}} \circ \theta_{j}<\infty\right\} .
$$

Hence, $\mathbf{P}_{x}\left(\bigcap_{n=1}^{\infty} \bigcup_{j=1}^{\infty}\left\{\sigma_{K_{n}} \circ \theta_{j}=\infty\right\}\right)=\mathbf{P}_{x}\left(\bigcap_{n=1}^{\infty} \bigcup_{j=1}^{\infty} \Lambda_{j}^{c}\right)=1$. In particular,

$$
\mathbf{P}_{x}\left(\bigcap_{n=1}^{\infty} \bigcup_{j=1}^{\infty}\left\{X_{t} \in E \backslash K_{n} \text { for all } t>j\right\}\right)=1
$$

for $x \in \mathbb{R}^{d}$. That is $\mathbf{P}_{x}\left(\lim _{t \rightarrow \infty}\left|X_{t}\right|=+\infty\right)=1$ for $x \in \mathbb{R}^{d}$. From this, $\mathbf{P}_{x}\left(\lim _{t \rightarrow \infty} u\left(X_{t}\right)=0\right)=1$ for $x \in \mathbb{R}^{d}$. On the other hand,

$$
\sup _{t>0} \mathbf{E}_{x}\left[\left(M_{t}^{u}\right)^{2}\right] \leq \mathbf{E}_{x}\left[\left\langle M^{u}\right\rangle_{\infty}\right]<\infty
$$

implies the uniform integrability of $\left(M_{t}^{u}\right)_{t \in[0, \infty[}$, which yields the existence of the limit $\lim _{t \rightarrow \infty} M_{t}^{u}$ under $\mathbf{P}_{x}$ for all $x \in \mathbb{R}^{d}$.

Take $\nu_{1}, \nu_{2} \in S_{D_{0}}^{1}(\mathbf{X}) \backslash S_{K}^{1}(\mathbf{X})$ and bounded symmetric functions $\phi_{1}, \phi_{2}$ on $\mathbb{R}^{d} \times \mathbb{R}^{d}$ vanishing on the diagonal. Suppose $\phi_{1}, \phi_{2} \in J_{D_{0}}^{1}(\mathbf{X})$. We set

$$
\begin{aligned}
& \mu_{1}=\frac{1}{2 c} \cdot \frac{\nu_{1}}{1+\left\|R\left(\nu_{1}+N\left(\phi_{1}\right) \mu_{H}\right)\right\|_{\infty}}, \\
& F_{1}=\log \left(1+\frac{1}{2 c} \cdot \frac{\phi_{1} e^{-U}}{1+\left\|R\left(\nu_{1}+N\left(\phi_{1}\right) \mu_{H}\right)\right\|_{\infty}}\right), \\
& \mu_{2}=\frac{1}{2 c} \cdot \frac{\nu_{2}}{1+\left\|R\left(\nu_{2}+N\left(\phi_{2}\right) \mu_{H}\right)\right\|_{\infty}}, \\
& F_{2}=\frac{1}{2 c} \cdot \frac{\phi_{2} e^{-U}}{1+\left\|R\left(\nu_{2}+N\left(\phi_{2}\right) \mu_{H}\right)\right\|_{\infty}},
\end{aligned}
$$

where $c$ is the constant appeared in the $3 G$-inequality. Then we see

$$
\begin{aligned}
\mu_{1}+N\left(e^{U}\left(e^{F_{1}}-1\right)\right) \mu_{H} & =\frac{1}{2 c} \cdot \frac{\nu_{1}+N\left(\phi_{1}\right) \mu_{H}}{1+\left\|R\left(\nu_{1}+N\left(\phi_{1}\right) \mu_{H}\right)\right\|_{\infty}} \\
& \in S_{\mathrm{CS}_{1}}^{1}(\mathbf{X}) \backslash S_{K}^{1}(\mathbf{X})
\end{aligned}
$$

and

$$
\mu_{2}+N\left(e^{U} F_{2}\right) \mu_{H}=\frac{1}{2 c} \cdot \frac{\nu_{2}+N\left(\phi_{2}\right) \mu_{H}}{1+\left\|R\left(\nu_{2}+N\left(\phi_{2}\right) \mu_{H}\right)\right\|_{\infty}} \in S_{\mathrm{DS}_{0}}^{1}(\mathbf{X}) \backslash S_{K}^{1}(\mathbf{X}) .
$$


Consider the following Feynman-Kac semigroup

$$
P_{t}^{A} f(x):=\mathbf{E}_{x}\left[e^{N_{t}^{u}+A_{t}^{\mu}+A_{t}^{F}} f\left(X_{t}\right)\right] .
$$

The associated quadratic form $\mathcal{Q}$ is given by

$$
\left\{\begin{aligned}
\mathcal{Q}(f, g):= & \int_{\mathbb{R}^{d} \times \mathbb{R}^{d}}(f(x)-f(y))(g(x)-g(y)) J_{m}(x, y) \mathrm{d} x \mathrm{~d} y \\
& +\int_{\mathbb{R}^{d} \times \mathbb{R}^{d}}(f g(x)-f g(y))(u(x)-u(y)) J_{m}(x, y) \mathrm{d} x \mathrm{~d} y \\
& -\int_{\mathbb{R}^{d}} f(x) g(x) \nu(\mathrm{d} x) \\
& -\int_{\mathbb{R}^{d} \times \mathbb{R}^{d}} f(x) g(y)\left(e^{F(x, y)}-1\right) J_{m}(x, y) \mathrm{d} x \mathrm{~d} y \\
\mathcal{D}(\mathcal{Q}):=\mathcal{F} . & \text { for } f, g \in \mathcal{D}(\mathcal{Q}) \cap L^{\infty}\left(\mathbb{R}^{d}\right),
\end{aligned}\right.
$$

In view of $\left[14\right.$, Theorem 1.2], the heat kernel $\check{p}_{t}^{U}(x, y)$ of the time changed process $\left(\check{\mathbf{U}}, \mathrm{d} x\right.$ ) obtained from the Girsanov transformed process $\mathbf{U}$ by $U_{t}=$ $\operatorname{Exp}\left(M^{e^{U}-1}\right)_{t}$ has the same global estimates as for the relativistic symmetric $\alpha$-stable process (symmetric $\alpha$-stable process if $m=0$ ). Note that the condition UJS in [14] is satisfied for (relativistic) symmetric $\alpha$-stable process (see [14, Example 2.3]). Then the Green kernel $R^{U}$ of $\mathbf{U}$, which coincides with the Green kernel $R^{\check{U}}$ of $(\check{\mathbf{U}}, \mathrm{d} x)$, satisfies (1.4) for some $C>0$. Then by Theorem 1.2, the following are equivalent:

(1) $\sup _{x \in \mathbb{R}^{d}} \mathbf{E}_{x}\left[e^{N_{\infty}^{u}+A_{\infty}^{\mu}+A_{\infty}^{F}}\right]<\infty$.

(2) For each $y \in \mathbb{R}^{d}, \sup _{x \in \mathbb{R}^{d} \backslash\{y\}} \mathbf{E}_{x}^{y}\left[\exp \left(N_{\zeta^{y}}^{u}+A_{\zeta^{y}}^{\mu}+A_{\zeta^{y}}^{F}\right)\right]<\infty$.

(3) $R^{u, \mu, F}(x, y)<\infty$ for all $x, y \in \mathbb{R}^{d}$ with $x \neq y$.

(4) $\lambda(u, \mu, F):=\inf \left\{Q(f, f) \mid f \in C_{0}^{\infty}\left(\mathbb{R}^{d}\right), \int_{\mathbb{R}^{d}} f(x)^{2} \bar{\mu}_{1}(\mathrm{~d} x)=1\right\}>0$.

Here $\bar{\mu}_{1}(\mathrm{~d} x):=\left(\int_{\mathbb{R}^{d}} V(x, y) J_{m}(x, y) \mathrm{d} y\right)(\mathrm{d} x)+\nu_{1}(\mathrm{~d} x)$ with $V(x, y):=\left(G^{u}-\right.$ $\left.F^{u}+F_{1}\right)(x, y)$ and $R^{u, \mu, F}(x, y)$ is the Green kernel of the Feynman-Kac semigroup by $N_{t}^{u}+A_{t}^{\mu}+A_{t}^{F}$. If $u=0, \mu_{1} \in S_{K_{\infty}}^{1}(\mathbf{X})$ and $e^{F_{1}}-1 \in A_{\mathrm{CS}_{1}}^{1}(\mathbf{X})$ (resp. $\mu_{1} \in S_{K_{\infty}}^{1}(\mathbf{X})$ and $\left.F_{1} \in A_{\mathrm{CS}_{\infty}}^{1}(\mathbf{X})\right)$, then we see $\left(\mu_{1}, e^{F_{1}}-1\right) \in B_{\mathrm{CS}_{1}}^{1}(\mathbf{X})$ (resp. $\left.\left(\mu_{1}, F_{1}\right) \in B_{\mathrm{CS}_{\infty}}^{1}(\mathbf{X})\right)$. In this case, (1)-(4) are equivalent to

(5) $\sup _{x, y \in \mathbb{R}^{d}, x \neq y} \mathbf{E}_{x}^{y}\left[e^{N_{\zeta^{y}}^{u}+A_{\zeta^{y}}^{\mu}+A_{\zeta^{y}}^{F}}\right]<\infty$.

If further $m>0$ and $\left(\mu_{2}, F_{2}\right) \in B_{\mathrm{DS}_{0}}^{1}(\mathbf{X}),(1)-(5)$ are equivalent to

(6) There exists $C>0$ such that

$$
C^{-1}\left(\frac{1}{|x-y|^{d-\alpha}}+\frac{1}{|x-y|^{d-2}}\right) \leq R^{u, \mu, F}(x, y) \leq C\left(\frac{1}{|x-y|^{d-\alpha}}+\frac{1}{|x-y|^{d-2}}\right)
$$


for all $x, y \in \mathbb{R}^{d}$ with $x \neq y$. If further $m=0$ and $\left(\mu_{2}, F_{2}\right) \in B_{\mathrm{DS}_{0}}^{1}(\mathbf{X}),(1)-(5)$ are equivalent to

(7) There exists $C>0$ such that

$$
\frac{C^{-1}}{|x-y|^{d-\alpha}} \leq R^{u, \mu, F}(x, y) \leq \frac{C}{|x-y|^{d-\alpha}} \quad \text { for all } x, y \in \mathbb{R}^{d} \text { with } x \neq y \text {. }
$$

\section{Maximum principle of generalized Feynman-Kac semigroups}

In this section, we apply Theorem 1.1 to prove the maximum principle of generalized Feynman-Kac semigroup extending the recent result [42] by Takeda. Throughout this section, we assume that $E$ is a locally compact separable metric space, $\mathfrak{m}$ is a positive Radon measure with full topological support and $\mathbf{X}$ is an $\mathfrak{m}$-symmetric process associated to a regular Dirichlet form $(\mathcal{E}, \mathcal{F})$ on $L^{2}(E ; \mathfrak{m})$ enjoying $(\mathbf{A C})$ and $(\mathbf{I})$. Let $u, \mu, F$ be as in the previous section and consider the generalized Feynman-Kac semigroup $P_{t}^{A} f(x)=\mathbf{E}_{x}\left[e_{A}(t) f\left(X_{t}\right)\right]$ with $e_{A}(t):=\exp \left(N_{t}^{u}+A_{t}^{\mu}+A_{t}^{F}\right)$. Let $\mathcal{S} \mathcal{H}^{\mathrm{ub}}(\mathcal{Q})$ be the family of upper bounded submedian function with respect to $\left(P_{t}^{A}\right)$, that is,

$\mathcal{S} \mathcal{H}^{\mathrm{ub}}(\mathcal{Q}):=\left\{h \in \mathcal{B}(E) \mid h\right.$ is upper bounded, $h \leq P_{t}^{A} h$ on $E$ for all $\left.t>0\right\}$ and define the maximum principle by If $h \in \mathcal{S} \mathcal{H}^{\mathrm{ub}}(\mathcal{Q})$, then $h(x) \leq 0$ for all $x \in E$.

Recall that $\mathbf{X}^{*}$ is the subprocess of $\mathbf{X}$ killed by $e^{-A_{t}^{\mu_{2}}-A_{t}^{F_{2}}}$. If $\mu_{2}+$ $N\left(F_{2}\right) \mu_{H} \in S_{1}(\mathbf{X})$ is non-trivial, then $\mathbf{X}^{*}$ is always transient under $(\mathbf{I})$.

THEOREM 7.1. Suppose that $\mu_{2}+N\left(F_{2}\right) \mu_{H} \in S_{1}(\mathbf{X})$ is non-trivial, $\bar{\mu}_{1}^{*} \in$ $S_{\mathrm{NK}_{1}}^{1}\left(\mathbf{X}^{*}\right)$ and $\mu_{\langle u\rangle} \in S_{D}^{1}(\mathbf{X}) \cap S_{\mathrm{NK}_{\infty}}^{1}\left(\mathbf{X}^{*}\right)$ hold. Assume

$$
\mathbf{E}_{x}\left[e^{-A_{\infty}^{\mu_{2}}-A_{\infty}^{F_{2}}}: \zeta=\infty\right]=0
$$

Then $\lambda^{\mathcal{Q}}\left(\bar{\mu}_{1}\right)>0$ implies (MP). Conversely, (MP) implies $\lambda^{\mathcal{Q}}\left(\bar{\mu}_{1}\right)>0$ provided $\mathbf{X}$ enjoys $(\mathbf{R S F})$, and $\mu_{1}+N\left(F_{1}\right) \mu_{H}+\mu_{\langle u\rangle} \in S_{K_{\infty}}^{1}\left(\mathbf{X}^{*}\right)$ and $\mu_{2}+$ $N\left(F_{2}\right) \mu_{H} \in S_{K}^{1}(\mathbf{X})$ hold.

Let us introduce the space $\mathcal{H}^{\mathrm{b}}(\mathcal{Q})$ of $\left(P_{t}^{A}\right)$-invariant bounded functions:

$$
\mathcal{H}^{\mathrm{b}}(\mathcal{Q}):=\left\{h \in \mathcal{B}_{b}(E) \mid h=P_{t}^{A} h \text { on } E \text { for all } t>0\right\}
$$

and define the Liouville property by

$$
\text { If } h \in \mathcal{H}^{\mathrm{b}}(\mathcal{Q}) \text {, then } h(x)=0 \text { for all } x \in E .
$$

Then we have the following.

Corollary 7.1. Suppose that $\mu_{2}+N\left(F_{2}\right) \mu_{H} \in S_{1}(\mathbf{X})$ is non-trivial, $\bar{\mu}_{1}^{*} \in$ $S_{\mathrm{NK}_{1}}^{1}\left(\mathbf{X}^{*}\right)$ and $\mu_{\langle u\rangle} \in S_{D}^{1}(\mathbf{X}) \cap S_{\mathrm{NK}_{\infty}}^{1}\left(\mathbf{X}^{*}\right)$ hold. Assume $(\mathbf{A})$. Then $\lambda^{\mathcal{Q}}\left(\bar{\mu}_{1}\right)>$ 0 implies $(\mathbf{L})$. 
When $\mathbf{X}$ is transient, the special cases of Theorem 7.1 and Corollary 7.1 are the following:

TheOREM 7.2. Suppose that $\mathbf{X}$ is transient, $\bar{\mu}_{1}^{*} \in S_{\mathrm{NK}_{1}}^{1}(\mathbf{X}), \mu_{\langle u\rangle} \in$ $S_{\mathrm{NK}_{\infty}}^{1}(\mathbf{X})$ and $\mu_{2}+N\left(F_{2}\right) \mu_{H} \in S_{D_{0}}^{1}(\mathbf{X})$ hold. Assume $(\mathbf{A})$. Then $\lambda^{\mathcal{Q}}\left(\bar{\mu}_{1}\right)>0$ implies (MP). Conversely, (MP) implies $\lambda^{\mathcal{Q}}\left(\bar{\mu}_{1}\right)>0$ provided $\mathbf{X}$ enjoys $(\mathbf{R S F})$, and $\mu_{1}+N\left(F_{1}\right) \mu_{H}+\mu_{\langle u\rangle} \in S_{K_{\infty}}^{1}(\mathbf{X})$ and $\mu_{2}+N\left(F_{2}\right) \mu_{H} \in S_{K}^{1}(\mathbf{X})$ hold.

Corollary 7.2. Suppose that $\mathbf{X}$ is transient, $\bar{\mu}_{1}^{*} \in S_{\mathrm{NK}_{1}}^{1}(\mathbf{X}), \mu_{\langle u\rangle} \in$ $S_{\mathrm{NK}_{\infty}}^{1}(\mathbf{X})$, and $\mu_{2}+N\left(F_{2}\right) \mu_{H} \in S_{D_{0}}^{1}(\mathbf{X})$ hold. Assume $(\mathbf{A})$. Then $\lambda^{\mathcal{Q}}\left(\bar{\mu}_{1}\right)>0$ implies $(\mathbf{L})$.

Note that under the conditions for measures in this section there exist positive constants $C, \alpha_{0}>0$ such that $\sup _{x \in E} P_{t}^{A} 1(x)=\sup _{x \in E} \mathbf{E}_{x}\left[e_{A}(t): t<\right.$ $\zeta] \leq C e^{\alpha_{0} t}$.

To prove Theorem 7.1, we need the following lemma.

LEMma 7.1. Suppose that $\mu_{2}+N\left(F_{2}\right) \mu_{H} \in S_{1}(\mathbf{X})$ is non-trivial and $\mu_{\langle u\rangle} \in$ $S_{1}(\mathbf{X}) \cap S_{\mathrm{NK}_{\infty}}^{1}\left(\mathbf{X}^{*}\right)$ holds. Then $\left(U_{t}\right)_{t \in[0, \infty[}$ is a uniformly integrable martingale under $\mathbf{P}_{x}^{*}$ for all $x \in E$.

Proof. Recall that $M_{t}:=M_{t}^{e^{U}-1}+M_{t}^{-u, c}$ is a locally square integrable local $\mathbf{P}_{x}$-martingale for all $x \in E$ under $\mu_{\langle u\rangle} \in S_{1}(\mathbf{X})$. By Itô formula for semimartingales, we see $M_{t}^{2}=2 \int_{0}^{t} M_{s-} \mathrm{d} M_{s}+[M]_{t}$, where $[M]_{t}=\left\langle M^{-u, c}\right\rangle_{t}+$ $\left[M^{e^{U}-1}\right]_{t}=\left\langle M^{-u, c}\right\rangle_{t}+\sum_{s \leq t}\left(e^{U\left(X_{s-}, X_{s}\right)}-1\right)^{2}$. We then have

$$
\sup _{x \in E} \mathbf{E}_{x}^{*}\left[[M]_{\infty}\right]=\sup _{x \in E} \mathbf{E}_{x}^{*}\left[\left\langle M^{-u, c}\right\rangle_{\infty}+\sum_{0 \leq t<\infty}\left(e^{U\left(X_{t-}, X_{t}\right)}-1\right)^{2}\right]<\infty
$$

under $\mu_{\langle u\rangle} \in S_{\mathrm{NK}_{\infty}}^{1}\left(\mathbf{X}^{*}\right) \subset S_{D_{0}}^{1}\left(\mathbf{X}^{*}\right)$, because $\left(e^{U}-1\right)^{2} \leq U^{2} e^{2\|u\|_{\infty}}$. From this

$$
\mathbf{E}_{x}^{*}\left[M_{t}^{2}\right]=\mathbf{E}_{x}\left[\int_{0}^{t} e^{-A_{s}^{\mu_{2}}-A_{s}^{F_{2}}} M_{s-} \mathrm{d} M_{s}\right]+\mathbf{E}_{x}^{*}\left[[M]_{t}\right]=\mathbf{E}_{x}^{*}\left[[M]_{t}\right]<\infty .
$$

Let $\left\{T_{n}\right\}$ be a increasing sequence of stopping times such that $\left(M_{t \wedge T_{n}}\right)_{t \in[0, \infty[}$ is a $\mathbf{P}_{x}$-martingale. Then

$$
\mathbf{E}_{x}^{*}\left[M_{t \wedge T_{n}}\right]=\mathbf{E}_{x}\left[\int_{0}^{t \wedge T_{n}} e^{-A_{s}^{\mu_{2}}-A_{s}^{F_{2}}} \mathrm{~d} M_{s}\right]=0 .
$$

This implies that $M_{t}$ is also a square integrable martingale under $\mathbf{P}_{x}^{*}$ for all $x \in E$. Combining (7.1) and (7.2), we have the uniform integrability of $\left(M_{t}\right)_{t \in[0, \infty[}$ under $\mathbf{P}_{x}^{*}$ for all $x \in E$ and the limit $M_{\infty}:=\lim _{t \rightarrow \infty} M_{t}$ exists $\mathbf{P}_{x}^{*}$-a.s. and in $L^{1}\left(\mathbf{P}_{x}^{*}\right)$ for all $x \in E$, consequently $\mathbf{E}_{x}^{*}\left[M_{\infty}\right]=0$. Applying $[6$, Theorem 3.2] to the square integrable $\mathbf{P}_{x}^{*}$-martingale $\left(M_{t}\right)_{t \in[0, \infty[}$ and $U_{t}=$ $\operatorname{Exp}(M)_{t}$ with $\Delta M_{t} \geq e^{-2\|u\|_{\infty}}-1$, we obtain the assertion. 
Proof of Theorem 7.1. Let $\mathbf{U}^{*}$ be the transformed process by $U_{t} e^{-A_{t}^{\mu_{2}}-A_{t}^{F_{2}}}$. Then $\mathbf{U}^{*}$ is also a transient $e^{-2 u}$ m-symmetric process. Under $\mu_{\langle u\rangle} \in$ $S_{\mathrm{NK}_{\infty}}^{1}\left(\mathbf{X}^{*}\right)$, any $\nu \in S_{\mathrm{NK}_{1}}^{1}\left(\mathbf{X}^{*}\right)$ implies $e^{-2 u} \nu \in S_{\mathrm{NK}_{1}}^{1}\left(\mathbf{U}^{*}\right)$ by Corollary 5.1. From this, $\bar{\mu}_{1}^{*}=\nu_{1}+N\left(e^{U}\left(e^{F_{1}}-1\right)\right) \mu_{H} \in S_{\mathrm{NK}_{1}}^{1}\left(\mathbf{X}^{*}\right) \quad$ yields $e^{-2 u} \nu_{1}+e^{-u} N\left(e^{-u}\left(e^{F_{1}}-1\right)\right) \mu_{H} \in S_{\mathrm{NK}_{1}}^{1}\left(\mathbf{U}^{*}\right)$. Consequently, $e^{-2 u} \nu_{1}+$ $e^{-u} N\left(e^{-u}\left(e^{-F_{2}}\left(e^{F_{1}}-1\right)\right)\right) \mu_{H} \in S_{\mathrm{NK}_{1}}^{1}\left(\mathbf{U}^{*}\right)$. Owing to the expression

$$
\begin{aligned}
\mathcal{Q}(f, g) & \\
= & \mathcal{E}^{U}\left(f e^{u}, g e^{u}\right)-\int_{E} f g \mathrm{~d} \bar{\nu}-\int_{E \times E} f(x) g(y)\left(e^{F(x, y)}-1\right) N(x, \mathrm{~d} y) \mu_{H}(\mathrm{~d} x) \\
= & \mathcal{E}^{U^{*}}\left(f e^{u}, g e^{u}\right)-\int_{E} f g \mathrm{~d} \bar{\nu}_{1} \\
& -\int_{E \times E} f(x) g(y)\left(e^{F_{1}(x, y)}-1\right) e^{-F_{2}(x, y)} N(x, \mathrm{~d} y) \mu_{H}(\mathrm{~d} x),
\end{aligned}
$$

we can deduce that $\lambda^{\mathcal{Q}}\left(\bar{\mu}_{1}^{*}\right)>0$ implies $\sup _{x \in E} \mathbf{E}_{x}^{U^{*}}\left[e^{A_{\zeta}^{\nu_{1}}+A_{\zeta}^{F_{1}}}\right]<\infty$ by applying Theorem 1.1 to $\mathbf{U}^{*}$ under $e^{-2 u} \nu_{1}+e^{-u} N\left(e^{-u}\left(e^{-F_{2}}\left(e^{F_{1}}-1\right)\right)\right) \mu_{H} \in$ $S_{\mathrm{NK}_{1}}^{1}\left(\mathbf{U}^{*}\right)$. Suppose $h \in \mathcal{S H}^{\mathrm{ub}}(\mathcal{Q})$ and $\lambda^{\mathcal{Q}}\left(\bar{\mu}_{1}\right)>0$. Then we have

$$
\begin{aligned}
h(x) & \leq P_{t}^{A} h(x)=e^{-u(x)} \mathbf{E}_{x}^{U}\left[e^{A_{t}^{\bar{\nu}}+A_{t}^{F}}\left(e^{u} h\right)\left(X_{t}\right)\right] \\
& =e^{-u(x)} \mathbf{E}_{x}^{U^{*}}\left[e^{A_{t}^{\bar{\nu}_{1}}+A_{t}^{F_{1}}}\left(e^{u} h\right)\left(X_{t}\right)\right] \\
& \leq e^{2\|u\|_{\infty}}\left\|h^{+}\right\|_{\infty} \mathbf{E}_{x}^{U^{*}}\left[e^{A_{\zeta}^{\bar{\nu}_{1}}+A_{\zeta}^{F_{1}}}: t<\zeta\right] .
\end{aligned}
$$

Under $\mu_{\langle u\rangle} \in S_{D}^{1}(\mathbf{X}),\left(U_{t}\right)_{t \in[0, \infty[}$ is a non-negative $\mathbf{P}_{x^{-}}$-supermartingale for all $x \in E$. Then there exists a limit $\bar{U}_{\infty}:=\lim _{t \rightarrow \infty} U_{t} \mathbf{P}_{x}$-a.s. On the other hand, Lemma 7.1 tells us that the convergence $\bar{U}_{\infty}:=\lim _{t \rightarrow \infty} U_{t}$ holds $\mathbf{P}_{x}^{*}$-a.s. and in $L^{1}\left(\mathbf{P}_{x}^{*}\right)$ for all $x \in E$. Then we have

$$
\begin{aligned}
\lim _{t \rightarrow \infty} \mathbf{P}_{x}^{U^{*}}(t<\zeta) & =\lim _{t \rightarrow \infty} \mathbf{E}_{x}^{*}\left[U_{t}: t<\zeta\right]=\mathbf{E}_{x}^{*}\left[\bar{U}_{\infty}: \zeta=\infty\right] \\
& =\mathbf{E}_{x}\left[\bar{U}_{\infty} e^{-A_{\infty}^{\bar{\nu}_{2}}-A_{\infty}^{F_{2}}} \mathbf{1}_{\{\zeta=\infty\}}\right]=0,
\end{aligned}
$$

because $e^{-A_{\infty}^{\bar{\nu}_{2}}-A_{\infty}^{F_{2}}} \mathbf{1}_{\{\zeta=\infty\}}=e^{-A_{\infty}^{\mu_{2}}-A_{\infty}^{F_{2}}} \mathbf{1}_{\{\zeta=\infty\}}=0 \quad \mathbf{P}_{x}$-a.s. for all $x \in E$ by $(\mathbf{A})$. Here we use $\mathbf{P}_{x}^{*} \ll \mathbf{P}_{x}$ on $\mathcal{F}_{\infty}$ with $\mathrm{d} \mathbf{P}_{x}^{*} / \mathrm{d} \mathbf{P}_{x}=\exp \left(-A_{\infty}^{\mu_{2}}-A_{\infty}^{F_{2}}\right)$ by [34, Theorem 1] (cf. [2, Lemma 1(1)] and [32, Lemma 2.3(a)]). Letting $t \rightarrow \infty$ in (7.3) with (7.4), we obtain $h(x) \leq 0$ for all $x \in E$. This yields (MP).

Next, we prove the converse assertion. Note that $\mathbf{X}^{*}$ enjoys $(\mathbf{R S F})$ and $S_{K}^{1}(\mathbf{X})=S_{K}^{1}\left(\mathbf{X}^{*}\right)$ under $\mu_{2}+N\left(F_{2}\right) \mu_{H} \in S_{K}^{1}(\mathbf{X})$. Indeed,

$$
\begin{aligned}
\lim _{t \rightarrow 0} \sup _{x \in E} \mathbf{E}_{x}\left[\left|e^{-A_{t}^{\mu_{2}}-A_{t}^{F_{2}}}-1\right|\right] & \leq \lim _{t \rightarrow 0} \sup _{x \in E} \mathbf{E}_{x}\left[A_{t}^{\mu_{2}}+A_{t}^{F_{2}}\right] \\
& =\lim _{t \rightarrow 0} \sup _{x \in E} \mathbf{E}_{x}\left[A_{t}^{\mu_{2}}+A_{t}^{N\left(F_{2}\right) \mu_{H}}\right]=0
\end{aligned}
$$


implies the (RSF) of $\mathbf{W}$ by [28, Corollary 5.1], and the estimate

$$
\begin{aligned}
\mathbf{E}_{x}\left[A_{t}^{\nu}\right] & =\mathbf{E}_{x}\left[\int_{0}^{t} e^{A_{s}^{\mu_{2}}+A_{s}^{F_{2}}} e^{-A_{s}^{\mu_{2}}-A_{s}^{F_{2}}} \mathrm{~d} A_{s}^{\nu}\right] \\
& \leq \mathbf{E}_{x}\left[e^{A_{t}^{\mu_{2}}+A_{t}^{F_{2}}} \int_{0}^{t} e^{-A_{s}^{\mu_{2}}-A_{s}^{F_{2}}} \mathrm{~d} A_{s}^{\nu}\right] \\
& =\left(\sup _{x \in E} \mathbf{E}_{x}\left[e^{2 A_{t}^{\mu_{2}}+2 A_{t}^{F_{2}}}\right]\right)^{1 / 2}\left(\sup _{x \in E} \mathbf{E}_{x}\left[\left(\int_{0}^{t} e^{-A_{s}^{\mu_{2}}-A_{s}^{F_{2}}} \mathrm{~d} A_{s}^{\nu}\right)^{2}\right]\right)^{1 / 2} \\
& \leq \sqrt{2} \sup _{x \in E} \mathbf{E}_{x}\left[e^{2 A_{t}^{\mu_{2}}+2 A_{t}^{F_{2}}}\right]^{\frac{1}{2}} \sup _{x \in E} \mathbf{E}_{x}^{*}\left[A_{t}^{\nu}\right]
\end{aligned}
$$

yields the inclusion $S_{K}^{1}\left(\mathbf{X}^{*}\right) \subset S_{K}^{1}(\mathbf{X})$, where we use

$$
\begin{aligned}
\sup _{x \in E} \mathbf{E}_{x}\left[e^{2 A_{t}^{\mu_{2}}+2 A_{t}^{F_{2}}}\right] & =\sup _{x \in E} \mathbf{E}_{x}\left[\operatorname{Exp}\left(A^{2 \mu_{2}}+A^{e^{2 F_{2}}-1}\right)\right] \\
& \leq \frac{1}{1-\sup _{x \in E} \mathbf{E}_{x}\left[A_{t}^{2 \mu_{2}}+A_{t}^{2^{2 F_{2}}-1}\right]}<\infty
\end{aligned}
$$

for small $t>0$ under $\mu_{2}+N\left(F_{2}\right) \mu_{H} \in S_{K}^{1}(\mathbf{X})$. Set $W_{t}:=Z_{t} e^{-A_{t}^{\mu_{2}}}=$ $Y_{t}^{1} e^{-A_{t}^{\mu_{2}}-A_{t}^{F_{2}}}$ and let $\mathbf{W}$ be the transformed process by $W_{t}$. It is easy to see that $\mathbf{W}$ also enjoys $(\mathbf{R S F})$ under $N\left(F_{1}\right) \mu_{H}+\mu_{\langle u\rangle} \in S_{K_{\infty}}^{1}\left(\mathbf{X}^{*}\right) \subset S_{K}^{1}\left(\mathbf{X}^{*}\right)$ and $\mu_{2}+N\left(F_{2}\right) \mu_{H} \in S_{K}^{1}(\mathbf{X})$. Indeed, applying [8, Lemma 3.2(iii)] with $N\left(F_{1}\right) \mu_{H}+\mu_{\langle u\rangle} \in S_{K}^{1}\left(\mathbf{X}^{*}\right)$, we have

$$
\limsup _{t \rightarrow 0} \mathbf{E}_{x \in E}^{*}\left[\left|Y_{t}^{1}-1\right|\right]=0 .
$$

This implies the (RSF) of $\mathbf{W}$ by [28, Corollary 5.1]. Moreover, we see that any $\nu \in S_{K}^{1}\left(\mathbf{X}^{*}\right)\left(\right.$ resp. $\left.\nu \in S_{K_{\infty}}^{1}\left(\mathbf{X}^{*}\right)\right)$ satisfies $\nu \in S_{K}^{1}(\mathbf{W})\left(\right.$ resp. $\nu \in S_{K_{\infty}}^{1}(\mathbf{W})$ ). Indeed, for $\nu \in S_{K}^{1}\left(\mathbf{X}^{*}\right)$ ans its PCAF $A_{t}^{\nu}$, we have the following estimate based on the martingale property of $Y_{t}^{1}:=\operatorname{Exp}\left(M^{e^{F_{1}+U}-1}+M^{-u, c}\right)_{t}$ with respect to $\mathbf{X}$ under $N\left(F_{1}\right) \mu_{H}+\mu_{\langle u\rangle} \in S_{K_{\infty}}^{1}\left(\mathbf{X}^{*}\right) \subset S_{K}^{1}\left(\mathbf{X}^{*}\right)=S_{K}^{1}(\mathbf{X})$ with [17, Lemma 2.2]:

$$
\begin{aligned}
\mathbf{E}_{x}^{W}\left[A_{t}^{\nu}\right] & =\mathbf{E}_{x}\left[\int_{0}^{t} Y_{s}^{1} e^{-A_{s}^{\mu_{2}}-A_{s}^{F_{2}}} \mathrm{~d} A_{s}^{\nu}\right] \\
& =\mathbf{E}_{x}\left[Y_{t}^{1} \int_{0}^{t} e^{-A_{s}^{\mu_{2}}-A_{s}^{F_{2}}} \mathrm{~d} A_{s}^{\nu}\right] \\
& \leq \mathbf{E}_{x}\left[\left(Y_{t}^{1}\right)^{2}\right]^{\frac{1}{2}} \sqrt{2} \sup _{y \in E} \mathbf{E}_{y}\left[\int_{0}^{t} e^{-A_{s}^{\mu_{2}}-A_{s}^{F_{2}}} \mathrm{~d} A_{t}^{\nu}\right] \\
& \leq \sup _{x \in E} \mathbf{E}_{x}\left[\left(Y_{t}^{1}\right)^{2}\right]^{\frac{1}{2}} \sqrt{2} \sup _{y \in E} \mathbf{E}_{y}^{*}\left[A_{t}^{\nu}\right] .
\end{aligned}
$$


Since $\sup _{x \in E} \mathbf{E}_{x}\left[\left(Y_{t}^{1}\right)^{2}\right] \rightarrow 1$ as $t \rightarrow 0$ under $N\left(F_{1}\right) \mu_{H}+\mu_{\langle u\rangle} \in S_{K_{\infty}}^{1}\left(\mathbf{X}^{*}\right) \subset$ $S_{K}^{1}\left(\mathbf{X}^{*}\right)=S_{K}^{1}(\mathbf{X})$, we have $\sup _{x \in E} \mathbf{E}_{x}^{W}\left[A_{t}^{\nu}\right] \rightarrow 0$ as $t \rightarrow 0$. Similarly

$$
\begin{aligned}
\mathbf{E}_{x}^{W}\left[\mathbf{1}_{K^{c}} * A_{t}^{\nu}\right] & =\mathbf{E}_{x}\left[\int_{0}^{t} Y_{s}^{1} e^{-A_{s}^{\mu_{2}}-A_{s}^{F_{2}}} \mathbf{1}_{K^{c}}\left(X_{s}\right) \mathrm{d} A_{s}^{\nu}\right] \\
& =\mathbf{E}_{x}\left[Y_{t}^{1} \int_{0}^{t} e^{-A_{s}^{\mu_{2}}-A_{s}^{F_{2}}} \mathbf{1}_{K^{c}}\left(X_{s}\right) \mathrm{d} A_{s}^{\nu}\right] \\
& \leq \mathbf{E}_{x}\left[\left(Y_{t}^{1}\right)^{2}\right]^{\frac{1}{2}} \sqrt{2} \sup _{y \in E} \mathbf{E}_{y}\left[\int_{0}^{t} e^{-A_{s}^{\mu_{2}}-A_{s}^{F_{2}}} \mathbf{1}_{K^{c}}\left(X_{s}\right) \mathrm{d} A_{t}^{\nu}\right] \\
& \leq \sup _{x \in E} \mathbf{E}_{x}\left[\left(Y_{t}^{1}\right)^{2}\right]^{\frac{1}{2}} \sqrt{2} \sup _{y \in E} \mathbf{E}_{y}^{*}\left[\mathbf{1}_{K^{c}} * A_{t}^{\nu}\right]
\end{aligned}
$$

implies $e^{-2 u} \nu \in S_{K_{\infty}}^{1}(\mathbf{W})$ for $\nu \in S_{K_{\infty}}^{1}\left(\mathbf{X}^{*}\right)$. Let $\left(\mathcal{E}^{W}, \mathcal{F}^{W}\right)$ be the Dirichet form on $L^{2}(E ; \mathfrak{m})$ associated to $\mathbf{W}$. Then we see

$$
\lambda^{\mathcal{Q}}\left(\bar{\mu}_{1}^{*}\right)=\inf \left\{\mathcal{E}^{W}(f, f)-1 \mid f \in \mathcal{F} \cap C_{0}(E), \int_{E} f^{2} \mathrm{~d} \bar{\mu}_{1}^{*}=1\right\} .
$$

Applying Stollmann-Voigt's inequality to $\left(\mathcal{E}^{Z}, \mathcal{F}^{Z}\right)$, we get

$$
\begin{aligned}
& \lambda^{\mathcal{Q}}\left(\bar{\mu}_{1}^{*}\right)+1=\inf \left\{\mathcal{E}^{Z}\left(f e^{u}, f e^{u}\right)+\int_{E} f^{2} \mathrm{~d} \mu_{2} \mid f \in \mathcal{F} \cap C_{0}(E), \int_{E} f^{2} \mathrm{~d} \bar{\mu}_{1}^{*}=1\right\} \\
& \geq \frac{1}{\left\|R^{Z}\left(e^{-2 u} \bar{\mu}_{1}^{*}\right)\right\|_{\infty}}>0 .
\end{aligned}
$$

Then one can get

$$
\inf \left\{\mathcal{E}^{Z}\left(f e^{u}, f e^{u}\right)+\int_{E} f^{2} \mathrm{~d} \mu_{2} \mid f \in \mathcal{F} \cap C_{0}(E), \lambda^{*} \int_{E} f^{2} \mathrm{~d} \bar{\mu}_{1}^{*}=1\right\}=1,
$$

where $\lambda^{*}:=\lambda^{\mathcal{Q}}\left(\bar{\mu}_{1}^{*}\right)+1$. Under $\lambda^{\mathcal{Q}}\left(\bar{\mu}_{1}^{*}\right) \leq 0$, we have $0<\lambda^{*} \leq 1$. Since $\mu_{1}+N\left(F_{2}\right) \mu_{H}+\mu_{\langle u\rangle} \in S_{K_{\infty}}^{1}\left(\mathbf{X}^{*}\right)$, we have $\bar{\mu}_{1}^{*} \in S_{K_{\infty}}^{1}\left(\mathbf{X}^{*}\right)$, hence $e^{-2 u} \bar{\mu}_{1}^{*} \in$ $S_{K_{\infty}}^{1}(\mathbf{W})$. By [41, Theorem 2.1], we can get the existence of the unique minimizer $v$ of (7.5). Set

$$
h(x):=\mathbf{E}_{x}^{W}\left[\int_{0}^{\zeta} v\left(X_{s}\right) \mathrm{d} A_{s}^{\lambda^{*} \bar{\mu}_{1}^{*}}\right] .
$$

As in [42, Section 5], we can deduce the strict positivity, boundedness and fine continuity of $h$ with respect to $\mathbf{W}$. Moreover, for each $t>0$ we have $h(x)=P_{t}^{W, \lambda^{*} \bar{\mu}_{1}^{*}} h(x)$ for q.e. $x \in E$. Hence, $P_{s}^{W, \lambda^{*} \bar{\mu}_{1}^{*}} h(x)=P_{t}^{W, \lambda^{*} \bar{\mu}_{1}^{*}} h(x)$ for all $x \in E$ and $t, s>0$. Letting $s \rightarrow 0$, we have $h(x)=P_{t}^{W, \lambda^{*} \mu_{1}^{*}} h(x)$ for all $x \in E$, because of the boundedness and fine continuity of $h$ under 
$\mathbf{W}$ and $\sup _{x \in E} \mathbf{E}_{x}^{W}\left[e^{A_{s_{0}}^{\lambda^{*} \bar{\mu}_{1}^{*}}}\right] \leq \sup _{x \in E} \mathbf{E}_{x}^{Z}\left[e^{A_{s_{0}}^{\bar{\mu}^{*}}}\right]=\sup _{x \in E} e^{u(x)} P_{s_{0}}^{A} e^{-u}(x) \leq$ $C e^{\alpha_{0} s_{0}}$ under $\lambda^{\mathcal{Q}}\left(\bar{\mu}_{1}^{*}\right) \leq 0$. Thus, $\lambda^{\mathcal{Q}}\left(\bar{\mu}_{1}^{*}\right) \leq 0$ yields

$$
\begin{aligned}
h(x) & =P_{t}^{W, \lambda^{*} \bar{\mu}_{1}^{*}} h(x) \leq P_{t}^{W, \bar{\mu}_{1}^{*}} h(x)=\mathbf{E}_{x}\left[Z_{t} e^{A_{t}^{\bar{\mu}^{*}}} h\left(X_{t}\right)\right] \\
& =\mathbf{E}_{x}\left[e_{A}(t) e^{u\left(X_{0}\right)-u\left(X_{t}\right)} h\left(X_{t}\right)\right]
\end{aligned}
$$

hence

$$
h e^{-u}(x) \leq P_{t}^{A}\left(h e^{-u}\right)(x),
$$

that is, $e^{-u} h$ is a positive element of $\mathcal{S} \mathcal{H}^{\mathrm{ub}}(\mathcal{Q})$. Therefore, (MP) does not hold under $\lambda^{\mathcal{Q}}\left(\bar{\mu}_{1}^{*}\right) \leq 0$.

Acknowledgment. The first and third authors would like to thank Professor Zhen-Qing Chen for suggesting the use of the transformation by $U_{t}$ and Professor Masayoshi Takeda for his valuable comments to the draft of this paper.

\section{REFERENCES}

[1] R. Azencott, Behavior of diffusion semi-groups at infinity, Bull. Soc. Math. France 102 (1974), 193-240. MR 0356254

[2] I. Ben-Ari and R. G. Pinsky, Absolute continuity/singularity and relative entropy properties for probability measures induced by diffusions on infinte time intervals, Stochastic Process. Appl. 115 (2005), 179-206. MR 2111192

[3] R. M. Blumenthal and R. K. Getoor, Markov processes and potential theory, Pure and Applied Mathematics, vol. 29, Academic Press, New York-London, 1968. MR 0264757

[4] Z.-Q. Chen, Gaugeability and conditional gaugeability, Trans. Amer. Math. Soc. 354 (2002), no. 11, 4639-4679. MR 1926893

[5] Z.-Q. Chen, Analytic characterization of conditional gaugeability for non-local Feynman-Kac transforms, J. Funct. Anal. 202 (2003), no. 1, 226-246. MR 1994771

[6] Z.-Q. Chen, Uniform integrability of exponential martingales and spectral bounds of non-local Feynman-Kac semigroups, Stochastic analysis and applications to finance, Interdiscip. Math. Sci., vol. 13, World Sci. Publ., Hackensack, NJ, 2012, pp. 55-75. MR 2986841

[7] Z.-Q. Chen and M. Fukushima, Symmetric Markov processes, time change, and boundary theory, London Mathematical Society Monographs Series, vol. 35, Princeton University Press, Princeton, NJ, 2012. MR 2849840

[8] Z.-Q. Chen and K. Kuwae, On doubly Feller property, Osaka J. Math. 46 (2009), no. 4, 909-930. MR 2604914

[9] Z.-Q. Chen and R. Song, General gauge and conditional gauge theorems, Ann. Probab. 30 (2002), no. 3, 1313-1339. MR 1920109

[10] Z.-Q. Chen and R. Song, Conditional gauge theorem for non-local Feynman-Kac transforms, Probab. Theory Related Fields 125 (2003), no. 1, 45-72. MR 1952456

[11] Z.-Q. Chen and R. Song, Drift transforms and Green function estimates for discontinuous processes, J. Funct. Anal. 201 (2003), no. 1, 262-281. MR 1986161

[12] Z.-Q. Chen and T.-S. Zhang, Girsanov and Feynman-Kac type transformations for symmetric Markov processes, Ann. Inst. Henri Poincaré B, Probab. Stat. 38 (2002), no. 4, 475-505. MR 1914937

[13] Z.-Q. Chen, P. J. Fitzsimmons, K. Kuwae and T.-S. Zhang, Stochastic calculus for symmetric Markov processes, Ann. Probab. 36 (2008), no. 3, 931-970. MR 2408579 
[14] Z.-Q. Chen, P. Kim and T. Kumagai, Global heat kernel estimates for symmetric jump processes, Trans. Amer. Math. Soc. 363 (2011), no. 9, 5021-5055. MR 2806700

[15] Z.-Q. Chen, P. Kim and R. Song, Global heat kernel estimates for relativistic stable processes in exterior open sets, J. Funct. Anal. 263 (2012), no. 2, 448-475. MR 2923420

[16] K. L. Chung and K. M. Rao, General gauge theorem for multiplicative functional functionals, Trans. Amer. Math. Soc. 306 (1988), no. 2, 819-836. MR 0933320

[17] G. De Leva, D. Kim and K. Kuwae, $L^{p}$-independence of spectral bounds of FeynmanKac semigroups by continuous additive functionals, J. Funct. Anal. 259 (2010), no. 3, 690-730. MR 2644101

[18] B. Fuglede, The quasi topology associated with a countably subadditive set function, Ann. Inst. Fourier (Grenoble) 21 (1971), no. 1, 123-169. MR 0283158

[19] M. Fukushima, Y. Oshima and M. Takeda, Dirichlet forms and symmetric Markov processes, 2nd revised and extended ed., de Gruyter Studies in Mathematics, vol. 19, Walter de Gruyter \& Co., Berlin, 2011. MR 2778606

[20] R.-K. Getoor and J. Glover, Riesz decompositions in Markov process theory, Trans. Amer. Math. Soc. 285 (1984), no. 1, 107-132. MR 0748833

[21] R. K. Getoor, Transience and recurrence of Markov processes seminar on probability, XIV (Paris, 1978/1979) (French), Lecture Notes in Math., vol. 784, Springer, Berlin, 1980. 397-409. MR 0580144

[22] R. K. Getoor, Gauge theorems for Stieltjes exponentials, Stochastic analysis and related topics, Advanced Studies in Pure Mathematics, vol. 41, 2004, pp. 91-109. MR 2083705

[23] I. W. Herbst and A. D. Sloan, Perturbation of translation invariant positivity preserving semigroups on $L^{2}\left(\mathbb{R}^{n}\right)$, Trans. Amer. Math. Soc. 236 (1978), 325-360. MR 0470750

[24] D. Kim and K. Kuwae, General analytic characterization of gaugeability for FeynmanKac functionals, to appear in Math. Ann.

[25] D. Kim and K. Kuwae, Stability of estimates for fundamental solutions under Feynman-Kac perturbations for symmetric Markov processes, preprint, 2016.

[26] D. Kim and K. Kuwae, Analytic characterizations of gaugeability for generalized Feynman-Kac functionals, to appear in Trans. Amer. Math. Soc.; available at http:// www.ams.org/cgi-bin/mstrack/accepted_papers/tran.

[27] D. Kim, K. Kuwae and Y. Tawara, Large deviation principle for generalized FeynmanKac functionals and its applications, Tohoku Math. J. 68 (2016), no. 2, 161-197. MR 3514698

[28] M. Kurniawaty, K. Kuwae and K. Tsuchida, On doubly Feller property of resolvent, to appear in Kyoto J. Math.

[29] K. Kuwae and M. Takahashi, Kato class measures of symmetric Markov processes under heat kernel estimates, J. Funct. Anal. 250 (2007), no. 1, 86-113. MR 2345907

[30] Y. Oshima, Semi-Dirichlet forms and Markov processes, de Gruyter Studies in Mathematics, vol. 48, Walter de Gruyter \& Co., Berlin, 2013. MR 3060116

[31] M. Ł. Ryznar, Estimates of green function for relativistic $\alpha$-stable process, Potential Anal. 17 (2002), no. 1, 1-23. MR 1906405

[32] R. Schilling and Z. Vondracek, Absolute continuity and singularity of probability measures induced by a purely discontinuous Girsanov transform of a stable process, to appear in Trans. Amer. Math. Soc.

[33] M. Sharpe, General theory of Markov processes, Academic Press, Boston, 1988. MR 0958914

[34] A. N. Shiryaev, Absolute continuity and singularity of probability measures in functional spaces, Proceedings of the international Congress of mathematicians (Helsinki, 1978), Acad. Sci. Fennica, Helsinki, 1980, pp. 209-225. MR 0562609 
[35] M. L. Silverstein, Symmetric Markov processes, Lect. Notes in Math., vol. 426, Springer, Berlin, 1974. MR 0386032

[36] P. Stollmann and J. Voigt, Perturbation of Dirichlet forms by measures, Potential Anal. 5 (1996), no. 2, 109-138. MR 1378151

[37] M. Takeda, Conditional gaugeability and subcriticality of generalized Schrödinger operators, J. Funct. Anal. 191 (2002), no. 2, 343-376. MR 1911190

[38] M. Takeda, Gaugeability for Feynman-Kac functionals with applications to symmetric $\alpha$-stable processes, Proc. Amer. Math. Soc. 134 (2006), no. 9, 2729-2738. MR 2213753

[39] M. Takeda, Some topics connected with gaugeability for Feynman-Kac functionals, Proceedings of RIMS workshop on stochastic analysis and applications, RIMS Kôkyûroku Bessatsu, B6, Res. Inst. Math. Sci., RIMS, Kyoto, 2008, pp. 221-236. MR 2407567

[40] M. Takeda, Feynman-Kac penalisations of symmetric stable processes, Electron. Commun. Probab. 15 (2010), 32-43. MR 2591632

[41] M. Takeda, A variational formula for Dirichlet forms and existence of ground states, J. Funct. Anal. 266 (2014), no. 1, 660-675. MR 3132724

[42] M. Takeda, The bottom of the spectrum of time-changed processes and the maximum principle of Schrödinger operators, preprint, 2015.

[43] M. Takeda and K. Tsuchida, Differentiability of spectral functions for symmetric $\alpha$ stable processes, Trans. Amer. Math. Soc. 359 (2007), no. 8, 4031-4054. MR 2302522

[44] M. Takeda and T. Uemura, Subcriticality and gaugeability for symmetric $\alpha$-stable processes, Forum Math. 16 (2004), no. 4, 505-517. MR 2044025

[45] Z. Zhao, Subcriticality and gaugeability of the Schrödinger operator, Trans. Amer. Math. Soc. 334 (1992), no. 1, 75-96. MR 1068934

Daehong Kim, Department of Mathematics and Engineering, Graduate School of Science and Technology, Kumamoto University, Kumamoto, 860-8555 Japan

E-mail address: daehong@gpo.kumamoto-u.ac.jp

Mila Kurniawaty, Department of Mathematics and Engineering, Graduate School of Science and Technology, Kumamoto University, Kumamoto, 860-8555 JAPAN

E-mail address: mila_akuwni@yahoo.com

Kazuhiro Kuwae, Department of Applied Mathematics, Faculty of Science, Fukuoka University, Fukuoka, 814-0180 JAPAN

E-mail address: kuwae@fukuoka-u.ac.jp 